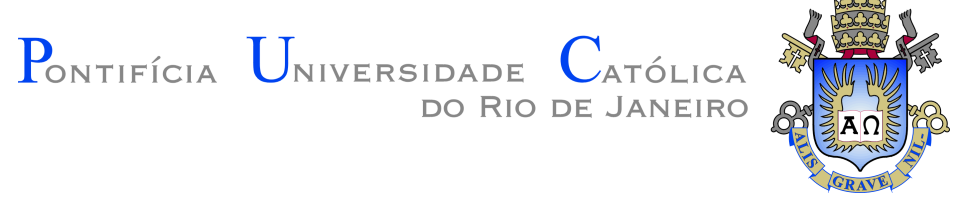

Renan Vieira Bela

\title{
Modeling Falloff Tests in Multilayered \\ Reservoirs
}

Dissertação de Mestrado

Thesis presented to the Programa de Pós-graduação em Matemática da PUC-Rio in partial fulfillment of the requirements for the degree of Mestre em Matemática .

Advisor

Prof. Sinésio Pesco

Co-advisor: Prof. Abelardo Borges Barreto Jr. 


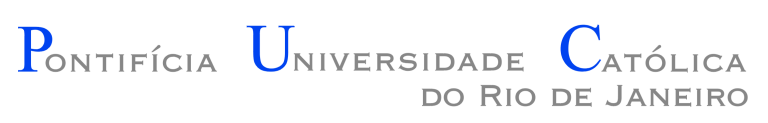

Renan Vieira Bela

\section{Modeling Falloff Tests in Multilayered \\ Reservoirs}

Thesis presented to the Programa de Pós-graduação em Matemática da PUC-Rio in partial fulfillment of the requirements for the degree of Mestre em Matemática. Approved by the undersigned Examination Committee.

Prof. Sinésio Pesco

Advisor

Departamento de Matemática - DMAT/PUC-Rio

Prof. Abelardo Borges Barreto Jr.

Co-advisor

Departamento de Matemática - PUC-Rio

Prof. Alvaro Marcello Marco Peres Laboratório de Engenharia e Exploração de Petróleo LENEP/UENF

Prof. Márcio da Silveira Carvalho

Departamento de Engenharia Mecânica - DEM/PUC-Rio

Dra. Priscila Magalhães Ribeiro

Centro de Pesquisa e Desenvolvimento Leopoldo Américo

Miguez de Mello - CENPES/Petrobras

Prof. Márcio da Silveira Carvalho

Vice Dean of Graduate Studies

Centro Técnico Científico - PUC-Rio

Rio de Janeiro, April the 13th, 2018 
All rights reserved.

\section{Renan Vieira Bela}

Graduated in Chemical Engineering from Pontífica Universidade Católica do Rio de Janeiro (PUC-Rio) in 2015. During graduation, worked for one year in a scientific initiation project, in the area of catalysts synthesis and characterization. Started the Mathematics Masters Program in PUC-Rio in 2016 and has been working since 2017 in the Oil \& Gas area, with focus on reservoir modeling.

Bibliographic data

Vieira Bela, Renan

Modeling Falloff Tests in Multilayered Reservoirs / Renan Vieira Bela; advisor: Sinésio Pesco; co-advisor: Abelardo Borges Barreto Jr.. - Rio de janeiro: PUC-Rio, Departamento de Matemática, 2018.

v., 75 f: il. color. ; $30 \mathrm{~cm}$

Dissertação (mestrado) - Pontifícia Universidade Católica do Rio de Janeiro, Departamento de Matemática.

Inclui bibliografia

1. Matemática - Teses. 2. Teste de Injetividade;. 3. Modelo Analítico;. 4. Período de Falloff;. 5. Reservatórios Multicamadas.. I. Pesco, Sinésio. II. Borges Barreto Jr., Abelardo. III. Pontifícia Universidade Católica do Rio de Janeiro. Departamento de Matemática. IV. Título. 


\section{Acknowledgments}

I would like to thank first my advisor Prof. Sinésio Pesco and my co-advisor Dr. Abelardo Barreto Jr. for the opportunity to learn with them, but, above all, for the patience and understanding with my questionings.

I also would like to thank my grandmother for the affection, for being my inspiration, the reason of all my achievements and because without her I certainly would not be here today.

I would like to thank my parents for the support and for encouraging me to apply for the masters program.

Last but not least important, I would like to thank CNPq and Petrobras for the financial support, PUC-Rio for the scholarship grant and Departamento de Matemática for the structure they let at my disposal. 


\section{Abstract}

Vieira Bela, Renan; Pesco, Sinésio (Advisor); Borges Barreto Jr., Abelardo (Co-Advisor). Modeling Falloff Tests in Multilayered Reservoirs. Rio de Janeiro, 2018. 75p. Dissertação de mestrado - Departamento de Matemática, Pontifícia Universidade Católica do Rio de Janeiro.

The injectivity test is a procedure used to collect information over a petroleum reservoir by injecting a fluid (commonly, water) into the reservoir. According to the pressure response measured during the test, several reservoir features might be inferred, such as equivalent permeability, outer boundary condition and recoverable oil volume. Injectivity test consists of two different stages: the flow period and the falloff period. During the former, occurs the water injection into the rock formation. The latter stage is marked by the well shut-in and, hence, a zero-flow pulse propagates along the reservoir. Over the past years, accomplishments have been made regarding the pressure behavior in multilayer reservoirs under single-phase flow and injectivity tests in single-layer reservoirs. However, an analytical solution for pressure behavior in multilayer reservoirs is well known just during the flow period. Therefore, this work attempts to develop an analytical model for the falloff period in multilayer reservoirs. The accuracy of the proposed solution was assessed by comparison with a finite difference flow simulator. Results showed a close agreement between the analytical model and numerical data. Moreover, falloff data obtained by the analytical solution was used to estimate the reservoir equivalent permeability. Calculated values presented a satisfactory accuracy for all cases.

\section{Keywords}

Injectivity Test; Multilayer Reservoir; Commingled System; Falloff Period; Analytical Model. 


\section{Resumo}

Vieira Bela, Renan; Pesco, Sinésio; Borges Barreto Jr., Abelardo.

Modelagem de Testes de Injetividade e Falloff em

Reservatórios Multicamadas. Rio de Janeiro, 2018. 75p. Dissertação de Mestrado - Departamento de Matemática, Pontifícia Universidade Católica do Rio de Janeiro.

O teste de injetividade é um procedimento que tem por objetivo extrair informações sobre um reservatório de petróleo a partir da injeção de um fluido (usualmente, água) no reservatório. Com base na resposta de pressão medida durante o tempo de teste, pode-se inferir uma série de características acerca do reservatório, tais como permeabilidade equivalente, condição de fronteira do reservatório e volume de óleo recuperável. O teste de injetividade é dividido em dois períodos: um de fluxo, durante o qual ocorre a injeção de água no reservatório; e o segundo de falloff, no qual o poço é fechado e o fluxo ao longo do reservatório cessa. Os modelos analíticos hoje existentes são capazes de descrever bem tanto o período de injeção quanto o de falloff apenas para reservatórios com uma camada. Nos reservatórios com múltiplas camadas, somente o período de injeção tem uma formulação conhecida. Portanto, esse trabalho tem como objetivo propor uma solução analítica para o período de falloff em reservatórios com múltiplas camadas. A precisão da solução proposta foi avaliada a partir da comparação com um simulador numérico de fluxo para uma série de casos. Os resultados mostraram boa concordância entre os dados numéricos e o modelo proposto. Além disso, os dados do falloff obtidos com a solução analítica foram usados para estimar a permeabilidade equivalente do reservatório. Os valores encontrados apresentaram precisão satisfatória para todos os casos.

\section{Palavras-chave}

Teste de Injetividade; Modelo Analítico; Período de Falloff; Reservatórios Multicamadas. 


\section{Table of contents}

$\begin{array}{lr}\text { List of figures } & 8\end{array}$

$\begin{array}{ll}\text { List of tables } & 10\end{array}$

1 Introduction 12

2 Previous Achievements 14

2.1 Modeling Single-Phase Flow In Multilayer Reservoirs 14

2.2 Modeling Injectivity Tests In Single-Layer Reservoirs 15

2.3 Modeling Injectivity Tests In Multilayer Reservoirs 17

3 Pressure Response During Injection $\quad 18$

3.1 Pressure Behavior In Single-Layer Reservoirs - Injection Period 19

3.2 Pressure Behavior In Multilayer Reservoirs - Injection Period 23

4 Pressure Response During Falloff $\quad 25$

4.1 Pressure Behavior In Single-Layer Reservoirs - Falloff Period 25

4.1.1 Calculating The Flow-Rate Of A Fluid Using The Source Line Approximation 28

4.2 Pressure Behavior In Multilayer Reservoirs - Falloff Period 29

4.3 Pressure Response In Reservoirs With Formation Damage 32

5 Results And Discussion $\quad 34$

5.1 Input Parameters 34

5.2 Cases Without Skin 37

5.3 Cases With Skin 41

5.4 Determining The Reservoir Equivalent Permeability 52

6 Conclusions And Suggestions For Future Works $\quad 57$

$\begin{array}{ll}\text { Bibliography } & 58\end{array}$

A Determining The Waterfront Shape $\quad 61$

A.1 Fractional Flow 61

A.2 Buckley-Leverett Theory For Cylindrical Geometry 63

A.3 Welge Method To Adjust The Waterfront Saturation Profile 65

B Pressure Response In Single-Layer Reservoirs Under Single-Phase Flow 68

C Pressure Response In Multilayer Reservoirs Under Single-Phase Flow $\quad 71$

$\begin{array}{ll}\text { D Superposition Principle } & 74\end{array}$ 


\section{List of figures}

Figure 3.1 Reservoir Model

Figure 4.1 Flow-Rate Profile Throughout The Reservoir In Three

Instants Of Time

Figure 4.2 Pressure Response From Two Wellbores, One With And

One Without Formation Damage

Figure 5.1 Relative Permeability Curves

Figure 5.2 Falloff Data For Case A1 Using Different Flow-rate Approximations

Figure 5.3 Pressure Response From Case A1 During Injection (Left)

And Falloff

Figure 5.4 Pressure Response From Case A2 During Injection (Left) And Falloff

Figure 5.5 Pressure Response From Case B1 During Injection And Falloff

Figure 5.6 Pressure Response From Case B2 During Injection And Falloff

Figure 5.7 Pressure Response From Case D1 During Injection And Falloff

Figure 5.8 Pressure Response From Case D2 During Injection And Falloff

Figure 5.9 Pressure Response From Case F1 During Injection And Falloff

Figure 5.10 Pressure Response From Case F2 During Injection And Falloff

Figure 5.11 Pressure Response From Case H1 During Injection And Falloff

Figure 5.12 Pressure Response From Case H2 During Injection And Falloff

Figure 5.13 Pressure Response From Case J1 During Injection And Falloff

Figure 5.14 Pressure Response From Case J2 During Injection And Falloff

Figure 5.15 Pressure Response From Case L1 During Injection And Falloff

Figure 5.16 Pressure Response From Case L2 During Injection And Falloff

Figure 5.17 Pressure Response From Case Q1 During Injection And Falloff

Figure 5.18 Pressure Response From Case Q2 During Injection And Falloff

Figure 5.19 Single-Phase Oil Flow For Case F1 During Injection (Left) And Falloff 
Figure 5.20 Single-Phase Oil Flow For Case F2 During Injection (Left) And Falloff

Figure 5.21 Single-Phase Oil Flow For Case H1 During Injection And Falloff

Figure 5.22 Single-Phase Oil Flow For Case H2 During Injection And Falloff

Figure 5.23 Single-Phase Oil Flow For Case J1 During Injection And Falloff

Figure 5.24 Single-Phase Oil Flow For Case J2 During Injection And Falloff

Figure 5.25 Single-Phase Oil Flow For Case L1 During Injection And Falloff

Figure 5.26 Single-Phase Oil Flow For Case L2 During Injection And Falloff

Figure 5.27 Single-Phase Oil Flow For Case Q1 During Injection And Falloff

Figure 5.28 Single-Phase Oil Flow For Case Q2 During Injection And Falloff

Figure 5.29 Semilog Graph For Case F1 - Numerical Data On The Left And Analytical Data On The Right

Figure 5.30 Semilog Graph For Case F2 - Numerical Data On The Left And Analytical Data On The Right

Figure 5.31 Semilog Graph For Case J1 - Numerical Data On The Left And Analytical Data On The Right

Figure 5.32 Semilog Graph For Case J2 - Numerical Data On The Left And Analytical Data On The Right

Figure A.1 Control Volume

Figure A.2 Cylindrical Control Volume

Figure A.3 Mobilities Profiles And Its Corresponding Fractional Flow Curve

Figure A.4 Fractional Flow Curve And Its Corresponding Waterfront Profile

Figure A.5 Fractional Flow Curve And Resulting Inconsistent Waterfront Profile

Figure A.6 Adjusted Fractional Flow Curve And Waterfront

Figure D.1 Flow History And Superposition Equivalent Scheme 


\section{List of tables}

Table 5.1 Tested Cases 36

Table 5.2 Equivalent Permeabilities Calculated Using IMEX Data 55

Table 5.3 Equivalent Permeabilities Computed Using Analytical Data 56 


\section{List of Abreviations}

$A$ - Cross sectional area

$B_{w}$ - Water formation volume factor

$c_{t}-$ Total compressibility

$f_{f}$ - Fractional flow of phase $f$

$h_{j}-$ Thickness of layer $j$

$I_{v}$ - Modified Bessel function of first kind and order $\mathrm{v}$

$K_{v}$ - Modified Bessel function of second kind and order $\mathrm{v}$

$k_{e q}-$ Reservoir equivalent permeability

$k_{j}$ - Total permeability in layer $j$

$k_{r f}$ - Relative permeability of phase $f$

$\hat{M}$ - Endpoint mobility ratio

$P$ - Pressure

$\bar{P}-$ Pressure in Laplace domain

$P_{w f}-$ Wellbottom hole pressure

$q_{j}$ - Total flow-rate in layer $j$

$q_{f_{j}}$ - Flow-rate in layer $j$ of phase $f$

$r$ - Radius

$r_{F_{j}}-$ Waterfront radius in layer $j$

$r_{w}-$ Wellbore radius

$S_{j}-$ Skin factor of layer $j$

$S_{w}$ - Water saturation

$t$ - Time

$t_{p}-$ Injection time

$u$ - Laplace variable

$v_{f}$ - Velocity of phase $f$

$\alpha_{p}$ - Pressure unit conversion constant

$\lambda_{f}$ - Mobility of phase $f$

$\hat{\lambda}_{f}-$ Endpoint mobility of phase $f$

$\phi$ - Porosity

$\mu_{f}-$ Viscosity of phase $f$

$\eta_{j}$ - Hydraulic diffusivity in layer $j$

$\rho_{f}-$ Density of phase $f$ 


\section{Introduction}

Whenever a petroleum reservoir is discovered, many of its features (such as permeability and extension) are not known with a high level of certainty. In order to determine such properties and, hence, the economical viability of the reservoir, there exists two main procedures. During a conventional production test, the wellbore establishes a pressure drop that results in oil flow from the reservoir to the surface. Although widely used, this test yields an oil volume that, according to brazilian laws, may not be sold in market and must be burn, raising its environmental impact.

For this reason, injectivity tests have been increasingly applied. As the production test, this procedure aims to evaluate, through the analysis of measured pressure data, reservoir productivity parameters, its extension and boundary conditions.

A typical injectivity test consists of two stages: the injection period and the falloff period. During the former, a fluid (usually water) is injected into the rock formation. Fluid injection will induce the displacement of the reservoir existing oil, causing a pressure rise. As the flooded area grows, a high water saturation region is formed around the wellbore. The latter stage is marked by the well shut-in and, thus, a zero-flow pulse propagates along the reservoir. As a result, measured pressure decreases.

Parameter estimation relies on the collected pressure data interpretation, both in a production or an injectivity test. According to the pressure profile and its derivative with respect to the logarithm of time, it is possible to estimate the reservoir equivalent permeability. The outer boundary condition is easily determined through characteristic signatures in the derivative. The existence of near-wellbore formation damage is also identifiable.

Past studies have formulated analytical models for both injection and falloff periods in single-layer reservoirs. However, injectivity test is applicable to reservoirs with more than one layer as well.

In multilayer reservoirs, layer flow-rate is not necessarily constant, since layers can communicate through the wellbore. Total flow-rate, on the other hand, remains constant, due to mass conservation. Furthermore, in some cases there might occur vertical flow between adjacent layers, owing to a nonzero 
vertical permeability. This phenomena is called formation crossflow.

Existing multilayer analytical models are able to fully describe pressure profile during production tests. Semilog graph techniques allow even the estimation of individual layer properties, provided the layer flow-rate history is known. For injectivity tests, however, a formulation only for the injection period is well defined. Falloff pressure behavior, in its turn, remains unknown.

In this context, the main purpose of this work is to develop an analytical model for falloff period in multilayer reservoirs. The suggested formulation was developed by combining the solution for injection period in multilayer reservoirs with the existing model for falloff period in single-layer reservoirs. Results were assessed through comparison with a finite-differences numerical simulator for a set of cases. Using conventional semilog techniques, the generated falloff data was used to estimate the reservoir equivalent permeability.

Following this introduction, chapter 2 shows an overview on the work that was previously done, regarding single-phase flow in multilayer systems and two-phase flow in single-layer reservoirs. The chapter ends by introducing the analytical model for injection period in multilayer reservoirs.

Chapter 3 describes the known formulation for injection period, both in single and multilayer systems. Then, in chapter 4 , the existing model for falloff period in single-layer reservoirs is presented. In the same chapter, the proposed formulation for falloff period in the multilayer case is developed.

The results achieved using the suggested model and the numerical simulator are shown in chapter 5. A comment on those results is made, and calculated equivalent permeabilities are also displayed. Lastly, chapter 6 consists of the conclusions that follow from the obtained results and provides suggestions for future works. 


\section{2}

\section{Previous Achievements}

Historically, transient pressure behavior in petroleum reservoirs has been modeled under two distinct lines of work. On one hand, analytical models for pressure response in multilayer reservoirs under single-phase flow have been developed. In parallel, the study on injectivity tests in single-layer reservoirs has been conducted.

Thus, this chapter will present a recap over the previous works conducted both on single-phase flow in multilayer systems and on two-phase flow in single-layer reservoirs. At the end, a comment is made about injectivity tests in multilayer systems.

\section{1}

\section{Modeling Single-Phase Flow In Multilayer Reservoirs}

Single-phase flow in multilayer reservoirs has been studied in the context of production tests, that is, when oil is removed from the reservoir. Nevertheless, the same equations also describe the pressure behavior during a theoretical injectivity test in which the injected fluid presents the same properties as the reservoir existing oil. Only adjustment required concerns the flow direction, that must occur from the wellbore to the reservoir, unlike a production test.

Lefkovits et al. (4) provided an analytical model for production tests in reservoirs with an arbitrary number of layers and no vertical flow between layers other than through the wellbore. Such reservoirs are denoted as commingled systems. Their model considers that layer properties such as thickness, permeability, formation damage and porosity may vary from one layer to another. Their results have been served as basis for many studies further developed.

Cobb et al. (5) conducted a comparison between three techniques for buildup data analysis in multilayer commingled reservoirs with equal layer thickness: Muskat, Miller-Dyes-Hutchinson and Horner methods. Buildup is the stage of a production test after the well is shut, equivalent to falloff in an injectivity test. They have shown that all three mentioned methods might be used to estimate the reservoir flow capacity, that is, the product between 
total thickness and equivalent permeability. Nevertheless, conventional semilog techniques were proven incapable of determining individual layer thickness or permeability.

Single-phase flow in reservoirs with two layers of distinct thickness was studied by Raghavan et al. (7). A means for obtaining individual layer permeability was proposed, assuming the thickness ratio is known. It was also shown that, among the techniques discussed by Cobb et al., Horner method is superior, as it allows the estimation of the reservoir average pressure and the ratio between layer permeabilities.

Gao (10) developed approximated solutions for pressure and flow-rate in each layer. The suggested formulation is able to provide estimatives for flow capacity in each layer. In the same work, there is also a discussion over the formation crossflow behavior and its effects.

A procedure for individual layer properties evaluation was proposed by Ehlig-Economides and Joseph (11). The suggested calculation is based on the analysis of pressure and layer flow-rate data. Permeability and formation damage for each layer may be determined through their method. In reservoirs with formation crossflow, even the vertical permeability between layers may be estimated.

Raghavan (14) conducted a review over the previous works about multilayer reservoirs. He described how differences in layer properties may lead to a pressure response distinct than the one of a single-layer equivalent reservoir. The influence of formation damage and crossflow were also reported. Furthermore, a technique to obtain individual layer properties from pressure and layer flow-rate data analysis was discussed.

Spath et al. (18) took advantage of the fact that layer flow-rates are decoupled when there is no formation crossflow and proposed a simple algorithm to compute wellbore pressure in commingled reservoirs. They proposed that layer flow-rate may assumed to be constant and determined using Duhamel's theorem. Thus, the single-layer solution may be used to calculate wellbore pressure in commingled systems.

\section{2}

\section{Modeling Injectivity Tests In Single-Layer Reservoirs}

The study on injectivity tests started to drain greater attention during the 80's. Abbaszadeh and Kamal (12) formulated an interpretation method for injectivity tests in single-layer reservoirs. Their solution accounts for relative permeability effects, formation damage and wellbore storage (the flow-rate difference between surface and reservoir due to fluid decompression). 
Abbaszadeh and Kamal proposed the waterfront saturation profile depicted by Buckley-Leverett (1) may be discretized into a series of banks. Thus, pressure behavior during an injectivity test would be equivalent to the one of a composite reservoir, formed by several distinct banks. They suggested that, during falloff, waterfront remains stationary, and proved the validity of such hypothesis under certain conditions. Thereby, type-curves for pressure and pressure derivative were developed.

Bratvold and Horne (16) evaluated the influence of temperature on fluid mobility and on saturation gradients. Their formulation was also based on the discretization of the saturation profile foreseen by Buckley-Leverett theory to obtain the pressure response. The model developed aims to generate type-curves for both injection and falloff, for the purpose of estimating reservoir properties such as storage effect, formation damage and the extension of the flooded region.

Oliveira and Serra (17) proposed a technique to estimate the relative permeability curves from falloff pressure data. Such calculation was made under the assumption that the relative permeability curves are approximated by polynomials whose coefficients are determined from the apparent skin foreseen by Bratvold and Horne's solution.

A general theory for radially heterogeneous reservoirs was developed by Thompson and Reynolds (19), either for single-phase or multiphase flow. They showed that pressure derivative may be understood as a weighted average of the permeabilities along the reservoir. The weighting factor is a function of flow-rate and mobility gradients. Therefore, regions where flow-rate and mobility changes are higher will be more relevant in the pressure derivative calculation. They stated that, in radially heterogeneous reservoirs, pressure data during the injection period show little resemblance with pressure data during falloff.

In a subsequent work, Banerjee et al. (20) modeled pressure behavior during injectivity tests in radially heterogeneous reservoirs. The same suggested formulation may be applied to homogeneous reservoirs as well. In such cases, a computation for the mechanical formation damage was developed.

A formulation for the injection period during injectivity tests in horizontal wells was presented by Peres and Reynolds (21). They considered the well is placed such that distance to the reservoir top and bottom boundaries are equal, and applied Thompson and Reynolds' steady-state theory. Thus, analytical expressions (that account for formation damage) for two of the three characteristic flow periods of a horizontal well testing were proposed - the third period occurs only in finite reservoirs, and such cases were not studied in their 
work.

The general theory for pressure behavior under water injection was presented by Peres et al. (22). They developed an analytical solution for the injection period and showed that their model reduces to the formulation proposed by previous authors, provided that the correct assumptions are made. Their solution is also capable of describing restricted entry wells and horizontal wells positioned at any point of the reservoir.

The falloff solution was reached by Peres et al. (24) following their own work (22). The superposition principle was used to describe the flow-rate history during falloff as the sum two flow-rates with different sign, but being applied at the same point of the reservoir.

\section{3}

\section{Modeling Injectivity Tests In Multilayer Reservoirs}

To the author's best knowledge, the first work regarding injectivity tests in multilayer reservoirs was presented by Barreto et al. (25). They applied Darcy's law to one given layer, so that an expression for the pressure change in this layer during the injection period is obtained. Thereby, layer flow-rate may be written as a function of that individual layer pressure change. A means for calculating wellbore pressure, then, is achieved by the sum of all layer flow-rates. Their solution for the injection period assumes no crossflow and radially infinite reservoir.

As disclosed in this overview, existing analytical models lack to describe falloff period in multilayer systems. Using the formulation developed by Barreto et al. (25) as basis, this work will attempt to extend the analytical model for falloff period in single-layer reservoirs (24) to multilayer systems as well. 


\section{3 \\ Pressure Response During Injection}

In this chapter, the formulation for single-layer reservoirs during the injection period, developed by Banerjee et al. (20) and Peres et al. (22), will be presented. This model will be extended to multilayer systems, as proposed by Barreto et al. (25). All calculations assume that a consistent unit set is used. The model developed considers the reservoir is subject to the following simplifying hypothesis:

- Reservoir in equilibrium at the instant $t=0$;

- Each layer presents constant thickness $h_{j}$;

- Homogeneous and isotropic reservoir, with infinite extension;

- Water and oil are assumed to be immiscible, slightly compressible fluids with constant viscosity $\mu$;

- Flow is isothermal;

- Rock formation presents a low and constant compressibility;

- Wellbore fully penetrates all layers and injects at constant flow-rate $q_{i n j}$;

- Gravitational and capillary forces are neglected;

- Initial pressure is the same for all layers;

- There is no wellbore storage;

- It is considered a commingled system, i.e., communication between layers happens only through the wellbore.

Reservoir may be composed by an arbitrary number of layers, which may present distinct formation damage zones, or even no formation damage. Oil viscosity, relative permeability curves and rock porosity may also vary according to the layer. However, for a matter of convenience, these properties were assumed to be the same in all layers during the calculations. Figure 3.1 shows the considered reservoir model. In this example, each layer is identified by the index 1,2 or $3 ; q_{j}, k_{j}$ and $h_{j}$ stand for the individual flow-rate, permeability and thickness in layer $j$, respectively. Subscript skin denotes the properties of the damaged zone (radius and permeability), which is only observed in the third layer. 


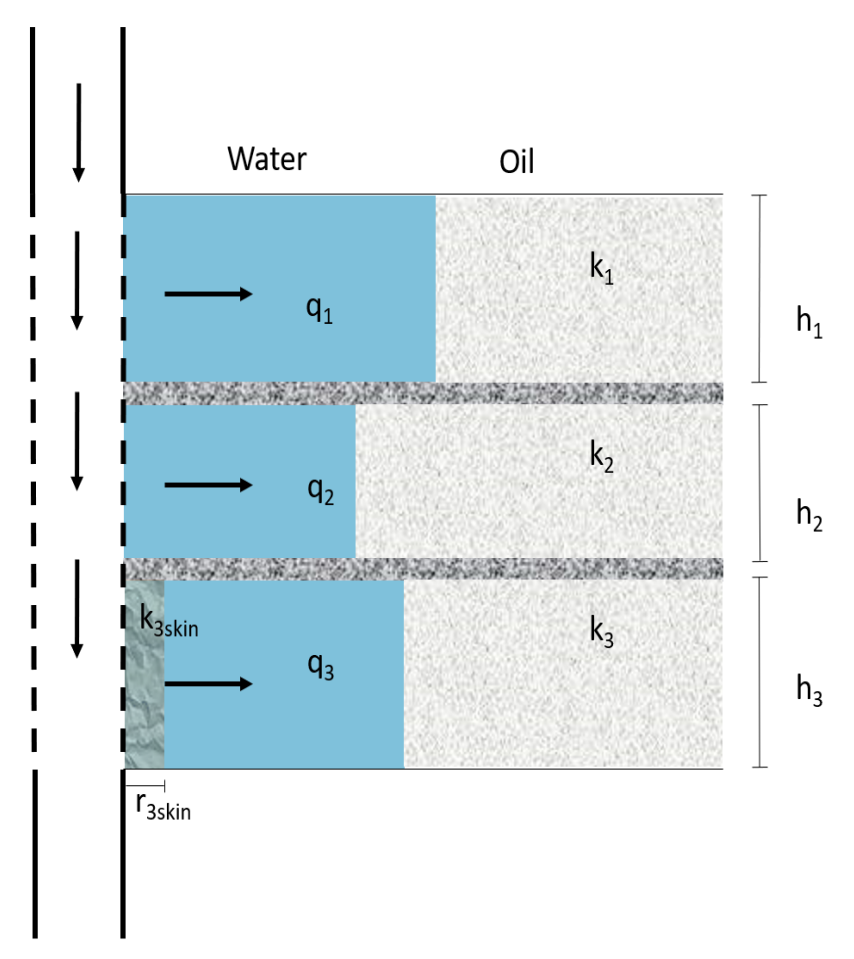

Figure 3.1: Reservoir Model

During the flow period, injected water displaces the reservoir oil, creating a high water saturation region around the wellbore. Beyond this region, oil flow occurs at its initial saturation. The idea is to split the wellbottom hole pressure in two components: one that encompasses the two-phase flow region and another that describes oil flow beyond the waterfront.

\section{1}

\section{Pressure Behavior In Single-Layer Reservoirs - Injection Period}

The starting point in order to compute the pressure variation in the reservoir during an injectivity test is Darcy's law $(20,21,25)$, that models flow through porous media. It states that flow-rate of a given fluid is calculated as:

$$
q_{f}(r, t)=-\frac{A k k_{r f}}{\mu_{f}} \frac{\partial P}{\partial r}
$$

where the $f$ index stands for the considered phase (water or oil), $A$ is the cross-sectional area, $k$ represents the media absolute permeability, $\mu_{f}$ stands for the fluid viscosity and $\frac{\partial P}{\partial r}$ denotes the pressure derivative with respect to the radius.

Moreover, $k_{r f}$ is the porous media relative permeability with respect to the phase considered. In other words, it is the percentage of the rock formation absolute permeability that is effectively felt by a fluid during its displacement. 
For a cylindrical geometry, Darcy's law may be rewritten as:

$$
q_{f}(r, t)=\frac{2 \pi h k k_{r f}}{\mu_{f}}\left(r \frac{\partial P}{\partial r}\right),
$$

where $h$ is the layer thickness.

Furthermore, due to the system mass conservation, it is valid to say that, inside the reservoir, total flow-rate is given by the sum of oil and water flow-rates:

$$
q(r, t)=q_{o}(r, t)+q_{w}(r, t)
$$

Which implies, by equations (3-2) and (3-3), that:

$$
q(r, t)=2 \pi h k\left(\frac{k_{r o}}{\mu_{o}}+\frac{k_{r w}}{\mu_{w}}\right) r \frac{\partial P}{\partial r}
$$

At this point, it is convenient to introduce another fluid property. The mobility of a phase measures the ability of this phase to flow through the porous media. It is defined as the ratio between the phase's relative permeability and its viscosity (19):

$$
\lambda_{f}\left(S_{w}\right)=\frac{k_{r f}\left(S_{w}\right)}{\mu_{f}},
$$

where $S_{w}$ stands for water saturation.

Relative permeability in the water-oil system is affected by water saturation along the porous media, since a higher water saturation impairs oil flow. Analogously, higher oil saturation impairs water displacement. Thus, relative permeability is a function of water saturation and, hence, so does the mobility.

During an injectivity test, a region with high water saturation is formed around the wellbore. At sufficiently large radius, water and oil remain at their connate saturations. These two zones are conected by a transition region, whose shape is defined by Buckley-Leverett theory, as shown in appendix A.

As injection goes on, the flooded area grows, and so does the high water saturation zone. Hence, water and oil saturation are functions of time and radius. As an immediate consequence, relative permeability and mobility will also depend on time and radius. Total mobility of the system in study is given by:

$$
\lambda_{T}=\lambda_{o}\left(S_{w}\right)+\lambda_{w}\left(S_{w}\right)=\lambda_{o}(r, t)+\lambda_{w}(r, t)=\lambda_{T}(r, t)
$$

Thereby, Darcy's law may be rearranged for the purpose of obtaining an expression for pressure change at any point of the reservoir as a function of the radius:

$$
\frac{\partial P}{\partial r}=\frac{q(r, t)}{2 \pi k h}\left(\frac{1}{\lambda_{o}+\lambda_{w}}\right) \frac{1}{r}=\frac{q(r, t)}{2 \pi k h} \frac{1}{\lambda_{T}(r, t)} \frac{1}{r}
$$


Thus, integrating both sides over the radius from the wellbore onwards:

$$
\int_{r_{w}}^{\infty} \frac{\partial P}{\partial r} d r=\int_{r_{w}}^{\infty} \frac{q(r, t)}{2 \pi k h} \frac{1}{\lambda_{T}(r, t)} \frac{d r}{r}
$$

where the wellbore radius is denoted by $r_{w}$.

The left side of equation (3-8) is evaluated by the Fundamental Theorem of Calculus, yielding:

$$
\int_{r_{w}}^{\infty} \frac{d P}{d r} d r=\lim _{r \rightarrow \infty} P(r, t)-P\left(r_{w}, t\right)=\lim _{r \rightarrow \infty} P(r, t)-P_{w f}(t),
$$

where $P_{w f}$ stands for the wellbottom hole pressure, that is, the pressure measured at the wellbore.

At a sufficiently large radius, the wellbore presence is no longer felt. Thus:

$$
\lim _{r \rightarrow \infty} P(r, t)=P_{i}
$$

where $P_{i}$ stands for the reservoir initial pressure.

On the right side of equation (3-8), only total mobility and total flow-rate are functions of the radius, since the porous media is assumed isotropic and homogeneous. Therefore, it is possible to obtain, for a given instant of time, a function relating pressure and radius (19):

$$
P_{i}-P_{w f}(t)=\Delta P_{w f}(t)=\frac{1}{2 \pi k h} \int_{r_{w}}^{\infty} \frac{q(r, t)}{\lambda_{T}(r, t)} \frac{d r}{r}
$$

Beyond the waterfront, only oil flows. On the other hand, behind the waterfront, a two-phase flow occurs $(12,16)$. Hence, it is logical to split the right side of equation (3-11) in two integrals, one whose integration limits go from the wellbore to the waterfront and another whose limits go from the waterfront onwards:

$$
\Delta P_{w f}(t)=\frac{1}{2 \pi k h}\left[\int_{r_{w}}^{r_{F}(t)} \frac{q(r, t)}{\lambda_{T}(r, t)} \frac{d r}{r}+\int_{r_{F}(t)}^{\infty} \frac{\hat{q}_{o}(r, t)}{\hat{\lambda}_{o}} \frac{d r}{r}\right]
$$

In the second integral of equation (3-12), total flow-rate was replaced by oil flow-rate at irreducible water saturation $\left(\hat{q}_{o}\right)$, and total mobility was replaced by the oil mobility at irreducible water saturation $\left(\hat{\lambda}_{o}\right)$. Those substitutions are possible due to the fact that, according to the reservoir model, oil is the only fluid flowing beyond the waterfront $(12,16)$. Furthermore, oil and water remain at the reservoir connate saturations beyond the waterfront, since injected water has not yet reached this region. Thus, oil flow-rate should be evaluated at its endpoint saturation. The waterfront radius, denoted by $r_{F}(t)$, is estimated by Buckley-Leverett theory, as discussed in appendix A.2. Adding and subtracting the term $\int_{r_{w}}^{r_{F}(t)} \frac{q(r, t)}{\hat{\lambda}_{o}} \frac{d r}{r}$ in equation (3-12): 


$$
\Delta P_{w f}(t)=\frac{1}{2 \pi k h} \int_{r_{w}}^{r_{F}(t)}\left(\frac{q(r, t)}{\lambda_{T}(r, t)}-\frac{\hat{q}_{o}(r, t)}{\hat{\lambda}_{o}}\right) \frac{d r}{r}+\frac{1}{2 \pi k h} \int_{r_{w}}^{\infty} \frac{\hat{q}_{o}(r, t)}{\hat{\lambda}_{o}} \frac{d r}{r}
$$

During the injection period, a non-zero flow-rate profile develops throughout the reservoir. Since flow-rate at the wellbore is constant, because injection flow-rate is constant by hypothesis, the region closest to the wellbore will also present constant flow-rate. At radii far from the wellbore, its presence is not felt and, hence, flow-rate at such radii will be zero. As the injection goes on, the constant non-zero flow-rate region expands.

It is known that the steady state region expands faster than the waterfront (19). Hence, both total and oil flow-rate behind the waterfront are constant and equal to the flow-rate at the interface between wellbore and reservoir.

This flow-rate is obtained through the adjustment of the surface injection flow-rate $\left(q_{i n j}\right)$ to reservoir conditions, and is given by the water formation volume factor $B_{w}$. Such adjustment is required since pressure and temperature conditions at the surface are different than the ones at the reservoir. Thereby, behind the waterfront, the following relation is valid:

$$
q(r, t)=\hat{q}_{o}(r, t)=q_{i n j} B_{w}
$$

Thus, equation (3-13) may be rewritten as:

$$
\Delta P_{w f}(t)=\underbrace{\frac{q_{i n j} B_{w}}{2 \pi k h} \int_{r_{w}}^{r_{F}(t)}\left(\frac{1}{\lambda_{T}(r, t)}-\frac{1}{\hat{\lambda}_{o}}\right) \frac{d r}{r}}_{\Delta P_{\lambda}(t)}+\underbrace{\frac{1}{2 \pi k h} \int_{r_{w}}^{\infty} \frac{\hat{q}_{o}(r, t)}{\hat{\lambda}_{o}} \frac{d r}{r}}_{\Delta P_{o}(t)}
$$

Equation (3-15) shows that the pressure variation at the wellbore may be understood as the sum of two parcels: one related to the single-phase oil flow $\left(\Delta P_{o}\right)$, and another owing to the mobility differences between oil and water $\left(\Delta P_{\lambda}\right)$ :

$$
\Delta P_{w f}(t)=\Delta P_{\lambda}(t)+\Delta P_{o}(t)
$$

where:

$$
\Delta P_{\lambda}(t)=\frac{q_{i n j} B_{w}}{2 \pi k h} \int_{r_{w}}^{r_{F}(t)}\left(\frac{1}{\lambda_{T}(r, t)}-\frac{1}{\hat{\lambda}_{o}}\right) \frac{d r}{r}
$$

and

$$
\Delta P_{o}(t)=\frac{1}{2 \pi k h} \int_{r_{w}}^{\infty} \frac{\hat{q}_{o}(r, t)}{\hat{\lambda}_{o}} \frac{d r}{r}
$$


A means for computing the term $\Delta P_{o}$ is shown in appendix $\mathrm{B}$. The result from equations (3-16) to (3-18) fully describes the pressure behavior during the injection period of an injectivity test in a single-layer reservoir.

\section{2}

\section{Pressure Behavior In Multilayer Reservoirs - Injection Period}

Darcy's law provides the flow-rate of a given phase along the porous media. This individual fluid displacement is decoupled with respect to each layer (18), assuming the oil remains immobile during water flow, and vice versa. Thus, for injectivity tests in multilayer reservoirs, Darcy's law may be applied in one given layer $j$, yielding:

$$
q_{f_{j}}(r, t)=-\frac{A k k_{r f_{j}}}{\mu_{f_{j}}} \frac{\partial P_{j}}{\partial r}
$$

Using an argument analogous to the one explained in section 3.1, an expression similar to equation (3-15) is obtained, modeling the pressure behavior in layer $j$ :

$$
\Delta P_{w f_{j}}(t)=\frac{q_{j}\left(r=r_{w}, t\right) B_{w}}{2 \pi k_{j} h_{j}} \int_{r_{w}}^{r_{F_{j}}(t)}\left(\frac{1}{\lambda_{T_{j}}(r, t)}-\frac{1}{\hat{\lambda}_{o_{j}}}\right) \frac{d r}{r}+\underbrace{\frac{1}{2 \pi k_{j} h_{j}} \int_{r_{w}}^{\infty} \frac{\hat{q}_{o j}(r, t)}{\hat{\lambda}_{o_{j}}} \frac{d r}{r}}_{\Delta P_{o_{j}}(t)}
$$

Under the assumptions made, fluids and relative permeability curves are the same in all layers. For this reason, all mobilities will be represented without any index from now on. Nevertheless, the proposed model is easily adapted to reservoirs where distinct layers present distinct relative permeability curves.

In appendix $\mathrm{C}$, it is shown that the term $\Delta P_{o_{j}}(t)$ is, in fact, the same for all layers and how it is computed. From now on, this term will be referred to as $\Delta P_{o}(t)$. Besides that, the term multiplying the layer flow-rate will be denoted as a weight variable $A_{j}(t)(25)$ :

$$
A_{j}(t)=\frac{B_{w}}{2 \pi k_{j} h_{j}} \int_{r_{w}}^{r_{F_{j}}(t)}\left(\frac{1}{\lambda_{T}(r, t)}-\frac{1}{\hat{\lambda}_{o}}\right) \frac{d r}{r}
$$

Moreover, under the assumptions made, pressure change is the same for all layers. Thus, pressure measured at the well bottom is equal to the pressure at any individual layer, except for the hydrostatic effect:

$$
\Delta P_{w f_{1}}(t)=\Delta P_{w f_{2}}(t)=\ldots=\Delta P_{w f_{n}}(t)=\Delta P_{w f}(t)
$$

Hence:

$$
\Delta P_{w f}(t)=q_{j}\left(r=r_{w}, t\right) A_{j}(t)+\Delta P_{o}(t)
$$


Thus, flow-rate in layer $j$ is given by:

$$
q_{j}\left(r=r_{w}, t\right)=\frac{\Delta P_{w f}(t)-\Delta P_{o}(t)}{A_{j}(t)}
$$

It is important to notice that layer flow-rate may change in time according to equation (3-24), although reservoir model assumes flow-rate remains constant at the wellbore. In fact, as there is no formation crossflow, the injected flow-rate will remain constant, and equal to the sum of flow-rates for all layers:

$$
q_{i n j}=\sum_{j=1}^{n} q_{j}\left(r=r_{w}, t\right)=\sum_{j=1}^{n} \frac{\Delta P_{w f}(t)-\Delta P_{o}(t)}{A_{j}(t)}
$$

Therefore:

$$
q_{i n j}=\left(\Delta P_{w f}(t)-\Delta P_{o}(t)\right) \sum_{j=1}^{n} A_{j}(t)^{-1}
$$

Then, it is possible to express the wellbore pressure in a way similar to expression (3-16):

$$
\Delta P_{w f}(t)=\Delta P_{o}(t)+\underbrace{\frac{q_{i n j}}{\sum_{j=1}^{n} A_{j}(t)^{-1}}}_{\Delta P_{\lambda}(t)}
$$

Thereby formulation for the injection period in a multilayer reservoir (25) is complete. For $n=1$, equation (3-27) reduces to equation (3-15), assuring the formulation is valid for reservoirs with any number of layers. 


\section{4 \\ Pressure Response During Falloff}

During falloff, pressure variation is calculated in a slightly different way than during the flow period:

$$
\Delta P_{w s}(\Delta t)=\Delta P_{w f}\left(t_{p}\right)-\Delta P_{w f}\left(t_{p}+\Delta t\right),
$$

where the subscript $s$ denotes the falloff period and $t_{p}$ stands for total injection time.

As defined in equation (3-16), pressure response during the injection period may be evaluated as the sum of two terms, one related to single-phase oil displacement and another that encompasses the mobility differences between water and oil. The goal is to apply the same idea during falloff and write an expression such as:

$$
\Delta P_{w s}=\Delta P_{o s}+\Delta P_{\lambda s}
$$

The term $\Delta P_{o s}$ is well known, and is computed by the superposition principle (see appendix D). The term $\Delta P_{\lambda s}$, on the other hand, was only well defined for single-layer reservoirs so far $(19,20)$, to the best of the author's knowledge. This work attempts to develop an expression that enables the calculation of $\Delta P_{\lambda s}$ during the falloff period even in multilayer reservoirs.

First, a means for evaluating $\Delta P_{\lambda s}$ in a single layer reservoir will be presented. Then, the resulting expression will be extended to a $n$-layer system. Same hypothesis made regarding the reservoir model used during the injection period are once again assumed during falloff.

\section{1}

\section{Pressure Behavior In Single-Layer Reservoirs - Falloff Period}

During falloff, a zero-rate pulse propagates throughout the reservoir. This means the steady state region formed during injection will be gradually replaced by a zero-rate zone. Figure 4.1 shows how the flow-rate behaves through the reservoir at the injection time $t_{p}$ and at two later instants of time.

Due to modeling for the injection period, it is valid to state that waterfront is always within the steady-state region (19, 20). Applying Darcy's 
law in a single layer reservoir, equation (3-11) is rewritten for the falloff period as:

$$
\Delta P_{w s}(\Delta t)=\frac{1}{2 \pi k h} \int_{r_{w}}^{\infty} \frac{q_{s}(r, \Delta t)}{\lambda_{T}(r, \Delta t)} \frac{d r}{r}
$$

where $q_{s}$ stands for the total flow-rate at the radius considered at any time after the well is shut and $\Delta P_{w s}$ is defined as in equation (4-1).

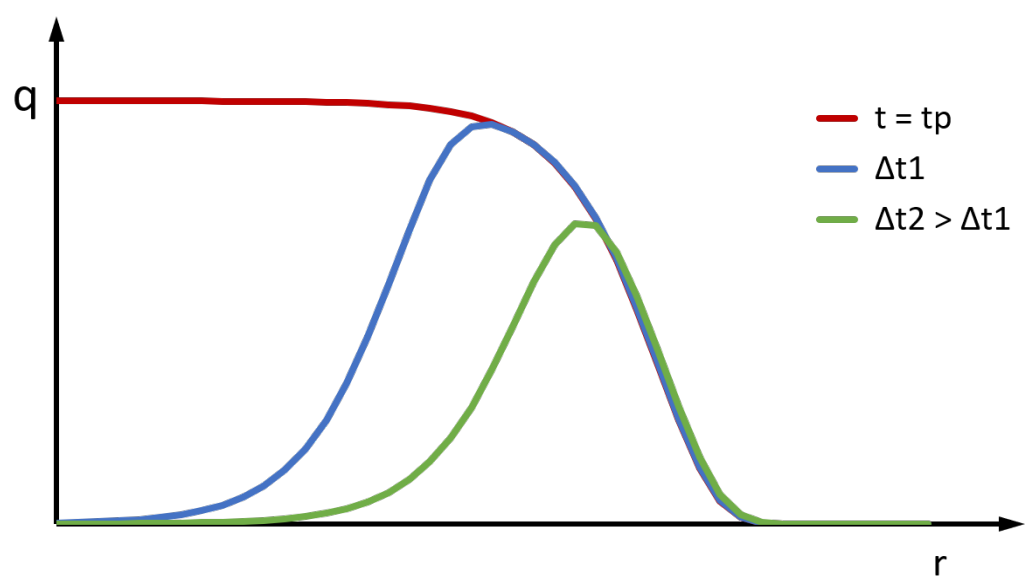

Figure 4.1: Flow-Rate Profile Throughout The Reservoir In Three Instants Of Time

It is known that the mobility profile remains practically constant during falloff $(12,16)$. This hypothesis, proved by empirical data, plays a crucial role to the computation of $\Delta P_{\lambda s}$. If the waterfront were transient, then flow-rate throughout the reservoir would also be transient, which would imply the pressure behavior could only be solved numerically.

Thus, total mobility in equation (4-3) depends only on the radius and the injection time $t_{p}$ :

$$
\Delta P_{w s}(\Delta t)=\frac{1}{2 \pi k h} \int_{r_{w}}^{\infty} \frac{q_{s}(r, \Delta t)}{\lambda_{T}\left(r, t_{p}\right)} \frac{d r}{r}
$$

By the same argument from section 3.2, behind the waterfront, water and oil are present in total flow-rate. Beyond the waterfront, total flow-rate consists only of oil flow-rate $\left(\hat{q}_{o s}\right)$, and total mobility in this region is the oil endpoint mobility $\left(\hat{\lambda}_{o}\right)$. Then, it is logical to split the integral in equation (4-4) in two different regions, the first whose limits go from the wellbore to the waterfront and the second whose limits go from the waterfront onwards:

$$
\Delta P_{w s}(\Delta t)=\frac{1}{2 \pi k h} \int_{r_{w}}^{r_{F}\left(t_{p}\right)} \frac{q_{s}(r, \Delta t)}{\lambda_{T}\left(r, t_{p}\right)} \frac{d r}{r}+\frac{1}{2 \pi k h} \int_{r_{F}\left(t_{p}\right)}^{\infty} \frac{\hat{q}_{o s}(r, \Delta t)}{\hat{\lambda}_{o}} \frac{d r}{r}
$$


where the waterfront radius at the injection time $\left(t=t_{p}\right)$ is, once again, determined according to Buckley-Leverett theory (see appendix A.2). Adding and subtracting the term $\int_{r_{w}}^{r_{F}\left(t_{p}\right)} \frac{\hat{q}_{o s}}{\hat{\lambda}_{o}} \frac{d r}{r}$ on the right side of equation (4-5):

$$
\Delta P_{w s}(\Delta t)=\underbrace{\frac{1}{2 \pi k h} \int_{r_{w}}^{r_{F}\left(t_{p}\right)}\left(\frac{q_{s}}{\lambda_{T}\left(r, t_{p}\right)}-\frac{\hat{q}_{o s}}{\hat{\lambda}_{o}}\right) \frac{d r}{r}}_{\Delta P_{\lambda s}(\Delta t)}+\underbrace{\frac{1}{2 \pi k h} \int_{r_{w}}^{\infty} \frac{\hat{q}_{o s}}{\hat{\lambda}_{o}} \frac{d r}{r}}_{\Delta P_{o s}(\Delta t)}
$$

Equation (4-6), proposed by Peres et al. (24), shows that pressure behavior during falloff may also be evaluated as the sum of one term related to the oil displacement and another that encompasses the mobility differences between the fluids. The single-phase contribution $\Delta P_{o s}(\Delta t)$ is evaluated as shown in appendix D.

The remaining question is how to estimate total flow-rate behind the waterfront. Two immediate rough approximations come from considering that total flow-rate is equal either to water or to oil flow-rate, calculated at their respective endpoint saturations:

$$
q_{s}(r, \Delta t)=\hat{q}_{o s}(r, \Delta t)
$$

Or:

$$
q_{s}(r, \Delta t)=\hat{q}_{w s}(r, \Delta t)
$$

One slightly more accurate approximation is to take an average between these two approximations, weighted by the water mobility at residual oil saturation and the oil mobility at irreducible water saturation:

$$
q_{s}(r, \Delta t)=\frac{\hat{\lambda}_{o} \hat{q}_{o s}(r, \Delta t)+\hat{\lambda}_{w} \hat{q}_{w s}(r, \Delta t)}{\hat{\lambda}_{o}+\hat{\lambda}_{w}}
$$

Approximation (4-9) is improved by taking in account the mobility profile along the flooded area:

$$
q_{s}(r, \Delta t)=\frac{\lambda_{o}(r) \hat{q}_{o s}(r, \Delta t)+\lambda_{w}(r) \hat{q}_{w s}(r, \Delta t)}{\lambda_{o}(r)+\lambda_{w}(r)}
$$

Finally, the most realistic way to determine total flow-rate would be to consider that even total compressibility $\left(c_{t}\right)$ varies with the radius:

$$
q_{s}(r, \Delta t)=\hat{q}_{\lambda s}\left(r, \Delta t, \lambda_{T}\left(r, t_{p}\right), c_{t}\left(r, t_{p}\right)\right)
$$

An estimation for water and oil flow-rates may be obtained using the source line approximation. 


\subsection{1}

\section{Calculating The Flow-Rate Of A Fluid Using The Source Line Approximation}

The comprehension of the procedure described in section 4.1 shows that an essential step consists of determining flow-rate at any point along the reservoir. Thus, equation (4-6) may be applied to compute the pressure change during falloff. Flow-rate at given time and radius is related to the pressure change according Darcy's law:

$$
q_{D}(r, \Delta t)=r_{D} \frac{\partial P_{D}(r, \Delta t)}{\partial r_{D}}
$$

where $P_{D}, t_{D}$ and $r_{D}$ are the dimensionless flow-rate, pressure change, and radius, defined as:

$$
q_{D}=\frac{q}{q_{i n j}} ; \quad P_{D}=\frac{k h \hat{\lambda}_{f} \Delta P}{q_{i n j} B_{f}} ; \quad r_{D}=\frac{r}{r_{w}},
$$

where the subscript $f$ indicates the flowing phase. Therefore, flow-rate may be immediately obtained once the pressure change is well defined, for example, like in equation (4-6). However, under the assumptions made, the source line approximation to compute the dimensionless pressure provides a quite accurate and much simpler estimate for the flow-rate. This model foresees that pressure change behaves as follows:

$$
P_{D}\left(r_{D}, t_{D}\right)=\frac{1}{2} \int_{x}^{\infty} \frac{\exp (-u)}{u} d u ; \quad x=\frac{r_{D}^{2}}{4 t_{D}},
$$

where $t_{D}$ is the dimensionless time, which is computed as follows:

$$
t_{D}=\frac{\hat{\lambda}_{f} k t}{\phi c_{t} r_{w}^{2}}
$$

In equation (4-15), $\phi$ denotes the formation porosity and $c_{t}$ stands for total compressibility.

The derivative of dimensionless pressure with respect to dimensionless radius is determined using Leibnitz's rule for differentiation under integral sign:

$$
\frac{\partial P_{D}}{\partial r_{D}}=-\frac{1}{r_{D}} \exp \left(\frac{-r_{D}^{2}}{4 t_{D}}\right)
$$

Thus, dimensionless flow-rate may be calculated through equations (4-12) and (4-16):

$$
q_{D}=\exp \left(\frac{-r_{D}^{2}}{4 t_{D}}\right)
$$

During falloff, pressure change is computed by applying the superposition principle, as depicted in appendix D. Then, total flow-rate should be evaluated as follows: 


$$
q_{D s}=r_{D} \frac{\partial P_{D s}(r, \Delta t)}{\partial r_{D}}=r_{D}\left(\frac{\partial P_{D}\left(r, t_{p}+\Delta t\right)}{\partial r_{D}}-\frac{\partial P_{D}(r, \Delta t)}{\partial r_{D}}\right)
$$

Hence:

$$
q_{D s}=\left[\exp \left(-\frac{r_{D}^{2}}{4 t_{D}}\right)-\exp \left(-\frac{r_{D}^{2}}{4 \Delta t_{D}}\right)\right]
$$

Oil flow-rate at a given radius is evaluated by applying equation (4-19) and using the oil properties to compute the dimensionless parameters. On the other hand, water flow-rate is calculated using the water properties to compute the dimensionless variables.

\section{2}

\section{Pressure Behavior In Multilayer Reservoirs - Falloff Period}

Now that the previously known analytical models are well understood, the same technique used by Barreto et al. (25) to develop their multilayer injection solution will be applied in order to reach a similar expression that depicts pressure behavior during falloff in multilayer reservoirs. Finally, the adjustments required when there is formation damage will be studied.

In a multilayer reservoir, the same argument depicted in section 4.1 shows that pressure change in one arbitrary layer $j$ is given by an expression similar to equation (4-6):

$$
\Delta P_{w s_{j}}(\Delta t)=\Delta P_{o s_{j}}(\Delta t)+\frac{1}{2 \pi k_{j} h_{j}} \int_{r_{w}}^{r_{F_{j}}\left(t_{p}\right)}\left(\frac{q_{s_{j}}(r, \Delta t)}{\lambda_{T}\left(r, t_{p}\right)}-\frac{\hat{q}_{o s_{j}}(r, \Delta t)}{\hat{\lambda}_{o}}\right) \frac{d r}{r}
$$

By hypothesis, there is no formation crossflow and pressure is the same in all layers. This means that, after the well is shut, the system is in equilibrium and there is no flow between layers, neither through the vertical boundaries between adjacent layers nor through the wellbore. This assumption is not only crucial to the development of the falloff formulation, but also significantly strong. Assuming that there is no flow between layers means, in other words, to state that vertical flow between layers either does not occur, or it is so small compared to the horizontal flow along the reservoir, that it may be neglected. This might not be true during falloff if layer properties are remarkably different (14).

From the negligible vertical flow hypothesis, follows that the zero-rate pulse propagates equally along all layers during falloff. Then, total and oil flow-rate may be expressed as functions of layer flow-rate fraction, which is 
the ratio between the flow-rate in a given layer $j$ and the sum of flow-rates in all layers, both evaluated at one given radius and one given instant of time:

$$
q_{D_{j}}(r, t)=\frac{q_{j}(r, t)}{q_{i n j}}
$$

To reach the expression for pressure change during falloff, flow-rate fraction in layer $j$ is assumed to remain constant within the flooded region after the well is shut, and equal to the flow-rate fraction at $t=t_{p}$. Then, total and oil flow-rates in layer $j$ may be expressed as:

$$
q_{s_{j}}(r, \Delta t)=q_{D p_{j}} q_{s}(r, \Delta t) ; \quad q_{o s_{j}}(r, \Delta t)=q_{D p_{j}} q_{o s}(r, \Delta t), \quad \text { if } r<r_{F_{j}}\left(t_{p}\right),
$$

where $q_{s}$ and $q_{o s}$ are computed as described in section $4.1 .1 ; q_{D p_{j}}$ is the layer flow-rate fraction just before shut-in, defined as:

$$
q_{D p_{j}}=\frac{q_{j}\left(t=t_{p}\right)}{q_{i n j}}
$$

Thus, equation (4-20) may be rewritten as:

$$
\Delta P_{w s_{j}}(\Delta t)=\Delta P_{o s_{j}}(\Delta t)+\frac{1}{2 \pi k_{j} h_{j}} \int_{r_{w_{j}}}^{r_{F_{j}}\left(t_{p}\right)} q_{D p_{j}}\left(\frac{q_{s}(r, \Delta t)}{\lambda_{T}\left(r, t_{p}\right)}-\frac{q_{o s}(r, \Delta t)}{\hat{\lambda}_{o}}\right) \frac{d r}{r}
$$

Since $q_{D p_{j}}$ is invariant with respect to radius, it may be written out of the integral:

$$
\Delta P_{w s_{j}}(\Delta t)=\Delta P_{o s_{j}}(\Delta t)+\frac{q_{D p_{j}}}{2 \pi k_{j} h_{j}} \int_{r_{w_{j}}}^{r_{F_{j}}\left(t_{p}\right)}\left(\frac{q_{s}(r, \Delta t)}{\lambda_{T}\left(r, t_{p}\right)}-\frac{q_{o s}(r, \Delta t)}{\hat{\lambda}_{o}}\right) \frac{d r}{r}
$$

The considered reservoir model states that pressure change is the same for all layers. So, once again, the pressure measured at the well bottom is equal to the pressure at any individual layer, apart from the hydrostatic effect:

$$
\Delta P_{w s_{1}}(\Delta t)=\Delta P_{w s_{2}}(\Delta t)=\ldots=\Delta P_{w s_{n}}(\Delta t)=\Delta P_{w s}(\Delta t)
$$

The single-phase contribution is also the same for every layers, as shown in appendices $\mathrm{C}$ and $\mathrm{D}$. Thus:

$$
\Delta P_{o s_{1}}(\Delta t)=\Delta P_{o s_{2}}(\Delta t)=\ldots=\Delta P_{o s_{n}}(\Delta t)=\Delta P_{o s}(\Delta t)
$$

Which means: 


$$
\Delta P_{w s}(\Delta t)-\Delta P_{o s}(\Delta t)=\frac{q_{D p_{j}}}{2 \pi k_{j} h_{j}} \int_{r_{w_{j}}}^{r_{F_{j}}\left(t_{p}\right)}\left(\frac{q_{s}(r, \Delta t)}{\lambda_{T}\left(r, t_{p}\right)}-\frac{q_{o s}(r, \Delta t)}{\hat{\lambda}_{o}}\right) \frac{d r}{r}
$$

Isolating $q_{D p_{j}}$ :

$$
q_{D p_{j}}=\frac{\Delta P_{w s}(\Delta t)-\Delta P_{o s}(\Delta t)}{\frac{1}{2 \pi k_{j} h_{j}} \int_{r_{w_{j}}}^{r_{F_{j}}\left(t_{p}\right)}\left(\frac{q_{s}(r, \Delta t)}{\lambda_{T}\left(r, t_{p}\right)}-\frac{q_{o s}(r, \Delta t)}{\hat{\lambda}_{o}}\right) \frac{d r}{r}}
$$

For any time step, in particular for $t=t_{p}$, the definition of layer flow-rate fraction ensures that:

$$
\sum_{j=1}^{n} q_{D_{j}}=\frac{1}{q_{i n j}} \sum_{j=1}^{n} q_{j}=1 \Rightarrow \sum_{j=1}^{n} q_{D p_{j}}=1
$$

Hence, from equations (4-29) and (4-30):

$$
1=\left(\Delta P_{w s}(\Delta t)-\Delta P_{o s}(\Delta t)\right) \sum_{j=1}^{n}\left(\frac{1}{\frac{1}{2 \pi k_{j} h_{j}} \int_{r_{w_{j}}}^{r_{F_{j}}\left(t_{p}\right)}\left(\frac{q_{s}(r, \Delta t)}{\lambda_{T}\left(r, t_{p}\right)}-\frac{q_{o s}(r, \Delta t)}{\hat{\lambda}_{o}}\right) \frac{d r}{r}}\right)
$$

Rearranging equation (4-31):

$$
\Delta P_{w s}(\Delta t)=\Delta P_{o s}(\Delta t)+\underbrace{\left(\sum_{j=1}^{n} \frac{1}{\frac{1}{2 \pi k_{j} h_{j}} \int_{r_{w_{j}}}^{r_{F_{j}}\left(t_{p}\right)}\left(\frac{q_{s}(r, \Delta t)}{\lambda_{T}\left(r, t_{p}\right)}-\frac{q_{o s}(r, \Delta t)}{\hat{\lambda}_{o}}\right) \frac{d r}{r}}\right)^{-1}}_{\Delta P_{\lambda s}}
$$

As described in equation (4-32), pressure change in multilayer reservoirs during falloff may be understood as the sum of the single-phase contribution with one term related to the mobility differences, in an analogous way to the injection period. Total and oil flow-rates are determined likewise the single-layer case (equations (4-7) through (4-11) and subsection 4.1.1).

At this point, another fundamental hypothesis is made. It was assumed that, in multilayer reservoirs, if the dimensionless parameters are calculated using the reservoir's total thickness and equivalent permeability, then the source line approximation yields the sum of oil (or water) flow-rate in all layers. Total thickness and equivalent permeability are defined as $(4,5)$ :

$$
\bar{h}=\sum_{j=1}^{n} h_{j} ; \quad k_{e q}=\frac{1}{\bar{h}} \sum_{j=1}^{n} k_{j} h_{j} 0
$$


Thereby, dimensionless time and pressure are calculated as follows:

$$
t_{D}=\frac{\hat{\lambda}_{f} k_{e q} t}{\phi c_{t} r_{w}^{2}} ; \quad P_{D}=\frac{k_{e q} \bar{h} \hat{\lambda}_{f} \Delta P}{q_{\text {inj }} B}
$$

Definitions for dimensionless radius and flow-rate remain the same as in the single-layer case.

\section{3}

\section{Pressure Response In Reservoirs With Formation Damage}

Perforation and completion of the wellbore may create a region with modified permeability $\left(k_{\text {skin }}\right)$ around the wellbore. This phenomena, known as formation damage or skin effect, results in an additional pressure rise, or drop, depending on the value $k_{\text {skin }}$.

Usually, formation damage impairs fluid displacement; that is, permeability in the damaged zone is smaller than reservoir permeability. Nevertheless, sometimes drilling process creates microfractures around the wellbore, stimulating the flow. In these cases, permeability in the damaged zone is higher than reservoir permeability. Figure 4.2 shows an example of the skin effect in two cases, one without formation damage and another where the damaged zone permeability is smaller than reservoir permeability.

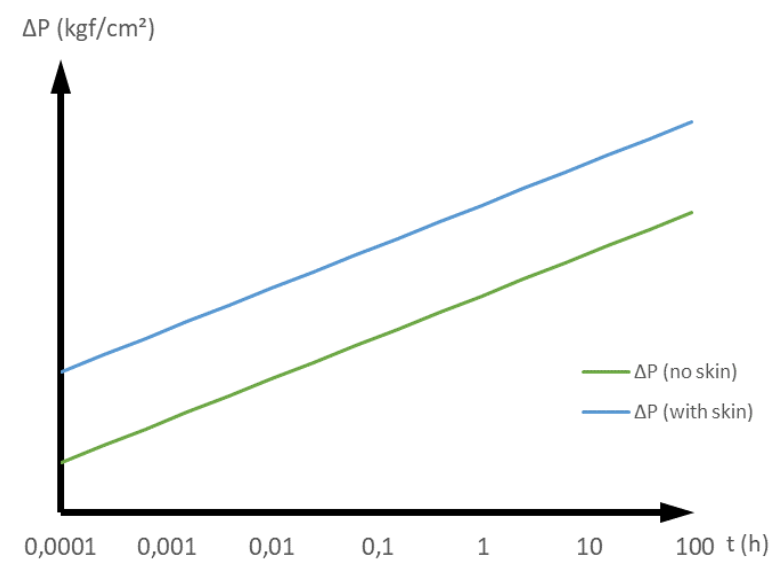

Figure 4.2: Pressure Response From Two Wellbores, One With And One Without Formation Damage

Thus, accounting for the skin effect, pressure at the wellbore during an injectivity test is computed as follows (16):

$$
\Delta P_{w f}=\Delta P_{\lambda}+\Delta P_{o}+\Delta P_{s k i n},
$$

The additional term $\Delta P_{\text {skin }}$ encompasses the effect of the damaged zone in the single-phase oil flow. It is calculated by (3): 


$$
\Delta P_{\text {skin }}=\frac{q_{i n j} B_{w}}{k h \hat{\lambda}_{o}} S
$$

where the term $S$ denotes Hawkins' skin factor. For single-layer reservoirs, it is defined as (3):

$$
S=\left(\frac{k}{k_{\text {skin }}}-1\right) \ln \left(\frac{r_{\text {skin }}}{r_{w}}\right)
$$

In multilayer reservoirs, the skin factor may be considered as the average of the individual layer skin factors weighted by their respective flow-rates (15):

$$
S=\frac{\sum_{j=1}^{n} q_{j} S_{j}}{q_{i n j}} ; \quad S_{j}=\left(\frac{k_{j}}{k_{j_{s k i n}}}-1\right) \ln \left(\frac{r_{j_{s k i n}}}{r_{w}}\right)
$$

The term $\Delta P_{\text {skin }}$ is computed using the endpoint oil mobility (equation (4-36)) because the effects of formation damage in the two-phase oil flow are accounted for inside the $\Delta P_{\lambda}$ term. The $A_{j}$ coefficient used in the calculation of $\Delta P_{\lambda}$ also must be adjusted owing to the presence of a damaged zone. The correction depends on the position of the waterfront compared to the skin radius. While the waterfront is within the damaged zone:

$$
A_{j}(t)=\frac{B_{w}}{2 \pi k_{j_{s k i n}} h_{j}} \int_{r_{w}}^{r_{F_{j}}(t)}\left(\frac{1}{\lambda_{T}}-\frac{1}{\hat{\lambda}_{o}}\right) \frac{d r}{r} \quad \text { if } r_{F_{j}}(t)<r_{j_{s k i n}}
$$

Otherwise, if the flooded region has overcome the damaged zone:

$$
A_{j}(t)=\frac{B_{w}}{2 \pi k_{j} h_{j}}\left[\int_{r_{w}}^{r_{F_{j}}(t)}\left(\frac{1}{\lambda_{T}}-\frac{1}{\hat{\lambda}_{o}}\right) \frac{d r}{r}+\left(\frac{k_{j}}{k_{j_{s k i n}}}-1\right) \int_{r_{w}}^{r_{j_{s k i n}}}\left(\frac{1}{\lambda_{T}}-\frac{1}{\hat{\lambda}_{o}}\right) \frac{d r}{r}\right]
$$

Skin effect is much more noticeable during the injection period than during falloff. That happens because the term $\Delta P_{\text {skin }}$ vanishes after the well is shut. Nevertheless, the formation damage is present during falloff in term $\Delta P_{\lambda s}:$

$$
\Delta P_{\lambda s}(t)=\left[\sum_{j=1}^{n} \frac{1}{\frac{1}{2 \pi k_{j} h_{j}}\left(\int_{r_{w}}^{r_{F_{j}}(t)}\left(\frac{q_{s}}{\lambda_{T}}-\frac{q_{o s}}{\hat{\lambda}_{o}}\right) \frac{d r}{r}+\left(\frac{k_{j}}{k_{j_{s k i n}}}-1\right) \int_{r_{w}}^{r_{j_{s k i n}}}\left(\frac{q_{s}}{\lambda_{T}}-\frac{q_{o s}}{\hat{\lambda}_{o}}\right) \frac{d r}{r}\right)}\right]^{-1}
$$




\section{5 \\ Results And Discussion}

In order to evaluate the accuracy of the solution proposed in section 4.2, a set of cases was run on the finite-difference based flow simulator IMEX. As an input for the numerical simulation, a radial grid was built. Radial discretization used increased the difference between two consecutive radial steps as the distance from the wellbore increases. Thereby, the grid is more refined in the region closest to the wellbore, that is, the region most affected by its presence. Minimum time step was set at $10^{-7} \mathrm{~h}$. Oil model used in IMEX is blackoil. The analytical model depicted in sections 4.2 and 4.3 was implemented in the open source software Scilab (28).

Pressure variation is expected to change in time as two semilog straight lines, one related to the oil properties and another governed by the water properties, separated by a transition period $(19,20)$.

So, in order to properly interpret the results of an injectivity test, one must analyze not only the pressure data, but also the pressure derivative with respect to the logarithm of time (from now on, referred to as pressure derivative). Each semilog straight line is identified by a period when constant pressure derivative, and reflects the properties of one distinct fluid (water or oil). Pressure derivative was numerically calculated for each case as proposed by Bourdet (13). Falloff pressure and pressure derivative data were plotted against Agarwal's equivalent time (9):

$$
t_{e q}=\frac{t_{p} \Delta t}{t_{p}+\Delta t}
$$

\section{1}

\section{Input Parameters}

For all cases, it was considered a 4 days (96 hours) injection period followed by a 4 days falloff period, as in a typical injectivity test. The 4 days injection time is short enough so that infinite radial regime is still acting (27). Injection flow-rate was defined as $500 \mathrm{~m}^{3} /$ day $\left(5.79 \times 10^{-3} \mathrm{~m}^{3} / \mathrm{s}\right)$, also an usual value for an injectivity test.

The hypothesis of negligible gravitational effects is valid for reservoirs with total thickness up to $50 \mathrm{~m}$. Thus, for all cases, total reservoir thickness 


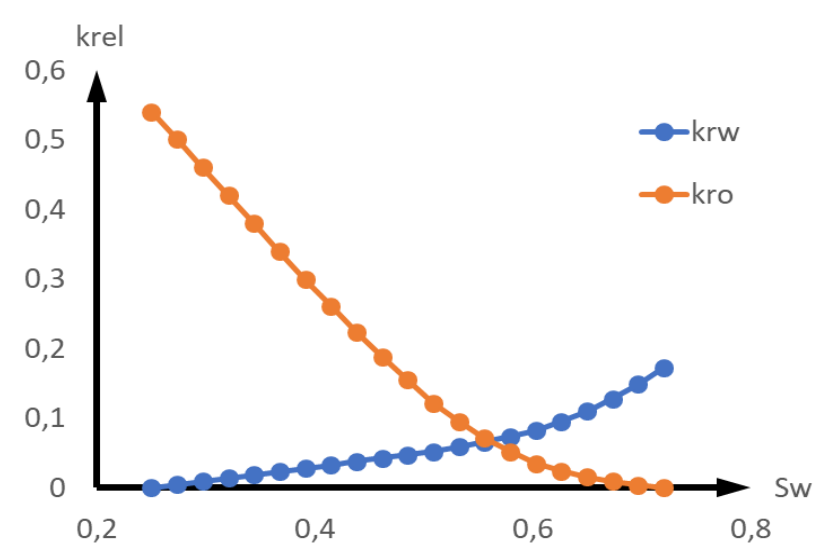

Figure 5.1: Relative Permeability Curves

was set as $30 \mathrm{~m}$, to ensure a safety margin with respect to the hypothesis validity range. Rock compressibility was assumed to be $8.00 \times 10^{-5}\left(\mathrm{kgf} / \mathrm{cm}^{2}\right)^{-1}$.

Water viscosity and compressibility were defined, respectively, as 0.52 cP $\left(5.2 \times 10^{-4}\right.$ Pa.s $)$ and $1.14^{-4}\left(\mathrm{kgf} / \mathrm{cm}^{2}\right)^{-1}$. Those are the usual values for water properties at a typical reservoir temperature $\left(50\right.$ to $\left.60{ }^{\circ} \mathrm{C}\right)$. In all multilayer cases, oil and relative permeability curves (displayed in figure 5.1) were assumed to be the same for every layer.

Depending on the relative easiness of water and oil to move through the reservoir, the two-phase flow may be labeled in two categories: favorable or unfavorable to water displacement. One useful variable to understand which fluid will move more easily is the endpoint mobility ratio $\hat{M}$. This parameter is physically meaningless, and comes from a variable used in reservoir engineering: the mobility ratio curve $M$, that consists of the ratio between two corresponding points in water and oil mobility curves:

$$
M\left(S_{w}\right)=\frac{\lambda_{w}\left(S_{w}\right)}{\lambda_{o}\left(S_{w}\right)}
$$

Ratio $\hat{M}$, then, is defined as the ratio between water mobility at residual oil saturation and oil mobility at initial water saturation:

$$
\hat{M}=\frac{\hat{\lambda}_{w}}{\hat{\lambda}_{o}}
$$

Flows favorable to water displacement imply that $\hat{M}>1$, while mobility ratios lower than 1 denote the flow is unfavorable to water displacement.

For each reservoir configuration, a pair of values for oil viscosity was chosen such that one would result in a flow favorable to water $\left(\mu_{o}=5.10 \mathrm{cP}\right)$ and the other would present a mobility ratio lower than $1\left(\mu_{o}=1.00 \mathrm{cP}\right)$. In all cases, oil compressibility was set as $4.04 \times 10^{-5}\left(\mathrm{kgf} / \mathrm{cm}^{2}\right)^{-1}$.

Table 5.1 shows the reservoir properties for each case. 
Table 5.1: Tested Cases

\begin{tabular}{|c|c|c|c|c|c|c|}
\hline Case & Layers & $\mathrm{k}(\mathrm{mD})$ & $\mathrm{h}(\mathrm{m})$ & $\mathrm{k}_{\text {skin }}(\mathrm{mD})$ & $\mathrm{r}_{\text {skin }}(\mathrm{m})$ & $\hat{\mathrm{M}}$ \\
\hline$\overline{\mathrm{A} 1}$ & 1 & 1000 & 30 & - & - & 3.09 \\
\hline A2 & 1 & 1000 & 30 & - & - & 0.61 \\
\hline $\mathrm{B} 1$ & 2 & 1000 (all) & 15 (all) & - & - & 3.09 \\
\hline B2 & 2 & 1000 (all) & 15 (all) & - & - & 0.61 \\
\hline D1 & 3 & $\begin{array}{c}1500 \\
500 \\
1000\end{array}$ & 10 (all) & - & - & 3.09 \\
\hline D2 & 3 & $\begin{array}{c}1500 \\
500 \\
1000\end{array}$ & 10 (all) & - & - & 0.61 \\
\hline F1 & 1 & 1000 & 30 & 500 & 0.5 & 3.09 \\
\hline $\mathrm{F} 2$ & 1 & 1000 & 30 & 500 & 0.5 & 0.61 \\
\hline H1 & 2 & 1000 (all) & 15 (all) & 500 (all) & 0.5 (all) & 3.09 \\
\hline $\mathrm{H} 2$ & 2 & 1000 (all) & 15 (all) & 500 (all) & 0.5 (all) & 0.61 \\
\hline J1 & 2 & 1000 (all) & 15 (all) & 500 and 100 & 0.5 (all) & 3.09 \\
\hline $\mathrm{J} 2$ & 2 & 1000 (all) & 15 (all) & 500 and 100 & 0.5 (all) & 0.61 \\
\hline L1 & 3 & 1000 (all) & 10 (all) & $\begin{array}{c}500 \\
500 \\
-\end{array}$ & $\begin{array}{c}0.5 \\
0.5 \\
-\end{array}$ & 3.09 \\
\hline L2 & 3 & 1000 (all) & 10 (all) & $\begin{array}{c}500 \\
500 \\
-\end{array}$ & $\begin{array}{c}0.5 \\
0.5 \\
-\end{array}$ & 0.61 \\
\hline Q1 & 5 & $\begin{array}{c}1000 \\
800 \\
1200 \\
900 \\
1400\end{array}$ & 6 (all) & $\begin{array}{l}500 \\
400 \\
600 \\
450 \\
700\end{array}$ & 0.5 (all) & 3.09 \\
\hline Q2 & 5 & $\begin{array}{c}1000 \\
800 \\
1200 \\
900 \\
1400\end{array}$ & 6 (all) & $\begin{array}{l}500 \\
400 \\
600 \\
450 \\
700\end{array}$ & 0.5 (all) & 0.61 \\
\hline
\end{tabular}




\section{2 \\ Cases Without Skin}

Cases A to D consist of reservoirs with no formation damage and same total thicknesses and equivalent permeabilities; that is, equal flow capacities. Thus, all cases from this set with the same mobility ratio are expected to present the same pressure response (14). Results for cases A to D are displayed in figures 5.3 to 5.8 .

Analytical solution and numerical data showed a good agreement for all cases, even for those with mobility ratio lower than 1 . When the flow is unfavorable to water, numerical simulation faces convergence issues due to the existence of shocks in the solution (25).

For all cases, it is noticeable that pressure derivative starts at a level during early time and, after a transition period, stabilizes at a different level at long time. This is a typical feature of a two-phase flow. During flow period, initially the injected water volume is small. Then, pressure behavior is mostly influenced by the oil properties. As the flooded region grows, water properties progressively prevail (23).

During falloff, the logic is reverse. At early times, pressure is governed by water properties. As the zero-rate pulse reaches the higher oil saturations regions, pressure derivative starts to reflect the properties of this fluid (23). For cases whose flow is favorable water, the highest pressure derivative level is associated to oil. That happens because the reservoir is more resistant to oil displacement than to water, which implies higher pressure variations during the time period the derivative is more influenced by oil properties. Similarly, for cases with $\hat{M}<1$, the highest derivative level is related to water. It is also important to keep in mind that, as defined in equation (4-1), pressure change increases during falloff.

For cases with $\hat{M}>1$, it is noticeable that the proposed formulation implies in pressure derivative values lower than the corresponding water level during falloff for $t_{e q}<0.001 \mathrm{~h}$. The main reason for such fact is the usage of the source line solution to determine total and oil flow-rates. This approximation becomes less accurate in the region closest to the wellbore, resulting in flow-rate values higher than expected.

Moreover, the choice of the approximation for total flow-rate is another cause of divergences between numerical and analytical data during early falloff times. Figure 5.2 compares the results obtained by the analytical model using approximations 1 to 4 (equations (4-7) to (4-10)). Approximation 5 (equation (4-11)) was not implemented, since its complexity-accuracy trade-off was not considered computationally worthy. Reservoir and fluids settings are the same 
as case A1. and only falloff period is shown (injection period was omitted, since it does not involve the mentioned flow-rate approximations). The pressure derivative level associated to water properties was identified through the results for the injection period.

Although approximation 1 (equation (4-7)) resulted in values lower than expected at early falloff times, the overall derivative behavior is consistent with a two-phase flow. On the other hand, approximations 2. 3 and 4 (equations (4-8) to (4-10)) yielded a pressure derivative profile incompatible with the physical reality, presenting an oscillatory region before the oil properties level is reached.

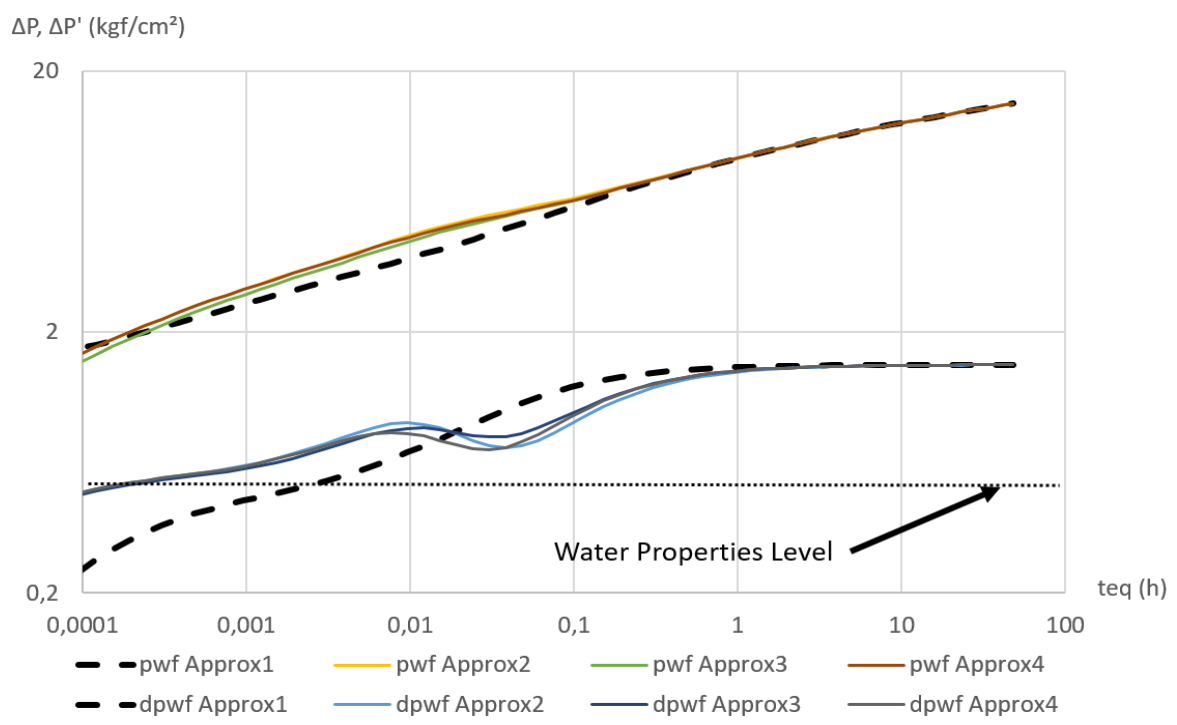

Figure 5.2: Falloff Data For Case A1 Using Different Flow-rate Approximations

Such unexpected fact is possibly related to the development of the formulation for the injection period. Equation (3-15) is achieved under the hypothesis that the flooded zone is within the steady state region. Hence, inside the waterfront, total flow-rate and oil flow-rate at irreducible water saturation are numerically equal during the injection period. Perhaps the results displayed in figure 5.2 reflect this assumption, suggesting that, during falloff, total flow-rate should be estimated by the oil flow-rate.

Besides the flow-rate approximation, another possible explanation for this lower derivative level is the accuracy of the Bessel functions used to evaluate $\Delta P_{o s}$ term. As depicted in appendices $\mathrm{C}$ and $\mathrm{D}$, computation of $\Delta P_{o s}$ involves modified Bessel functions $K_{0}$ and $K_{1}$. For very small time arguments, the imprecision associated to the computational calculation of those functions becomes more relevant. 
Thus, initial falloff times are subject to an error that is inherent to the flow-rate approximation and computational issues, but not related to the hypothesis required to reach equation (4-32) Despite that, as the zero-rate pulse propagates through the reservoir, analytical solution and numerical data rapidly converge.

As expected, cases with the same mobility ratio presented almost identical pressure responses, during both injection and falloff. This is consistent with the fact that flow capacity is the same for cases A to D.

Cases A1 and A2 are two reference cases. They show the pressure response in a single-layer reservoir, which is already known. Case A1 depicts a flow that is favorable to water, while case A2 presents an endpoint mobility ratio smaller than 1 .

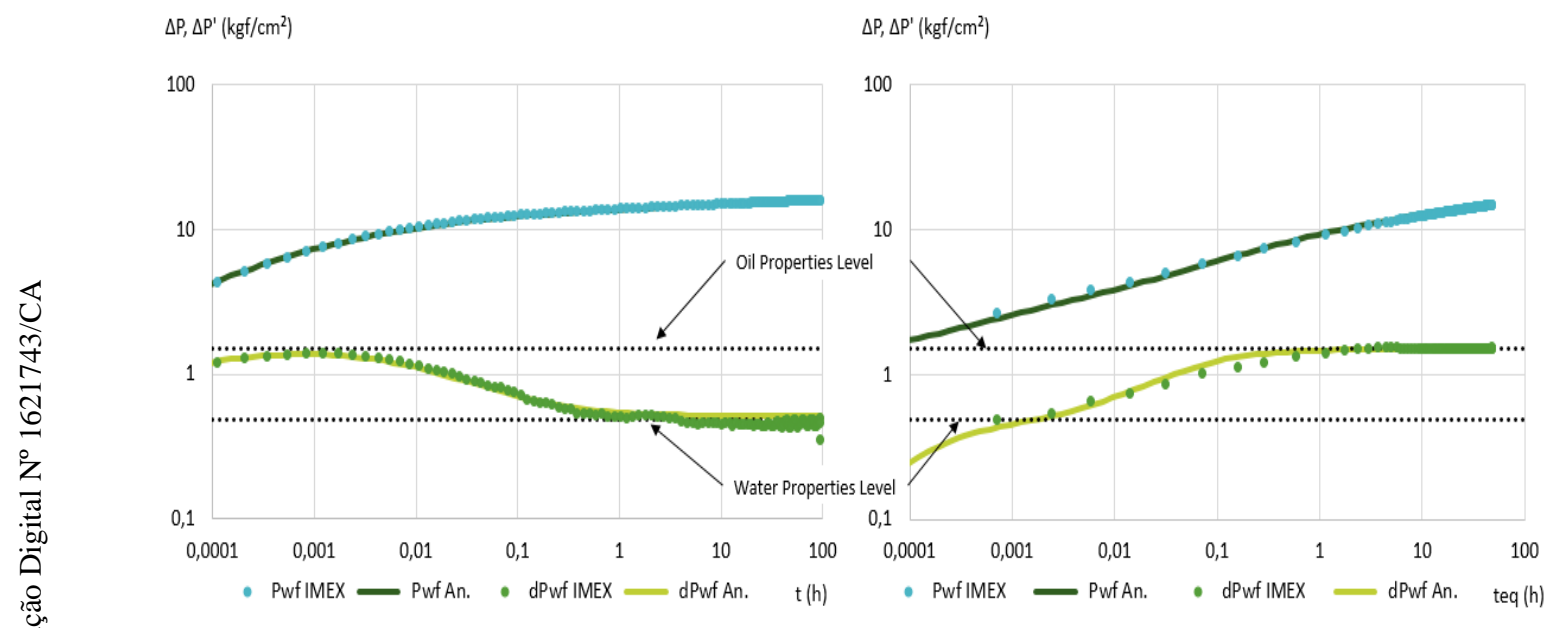

Figure 5.3: Pressure Response From Case A1 During Injection (Left) And Falloff

Cases B1 and B2 stand for reservoirs with same flow capacity as the reference cases, but with two layers, both of them with the same properties. 


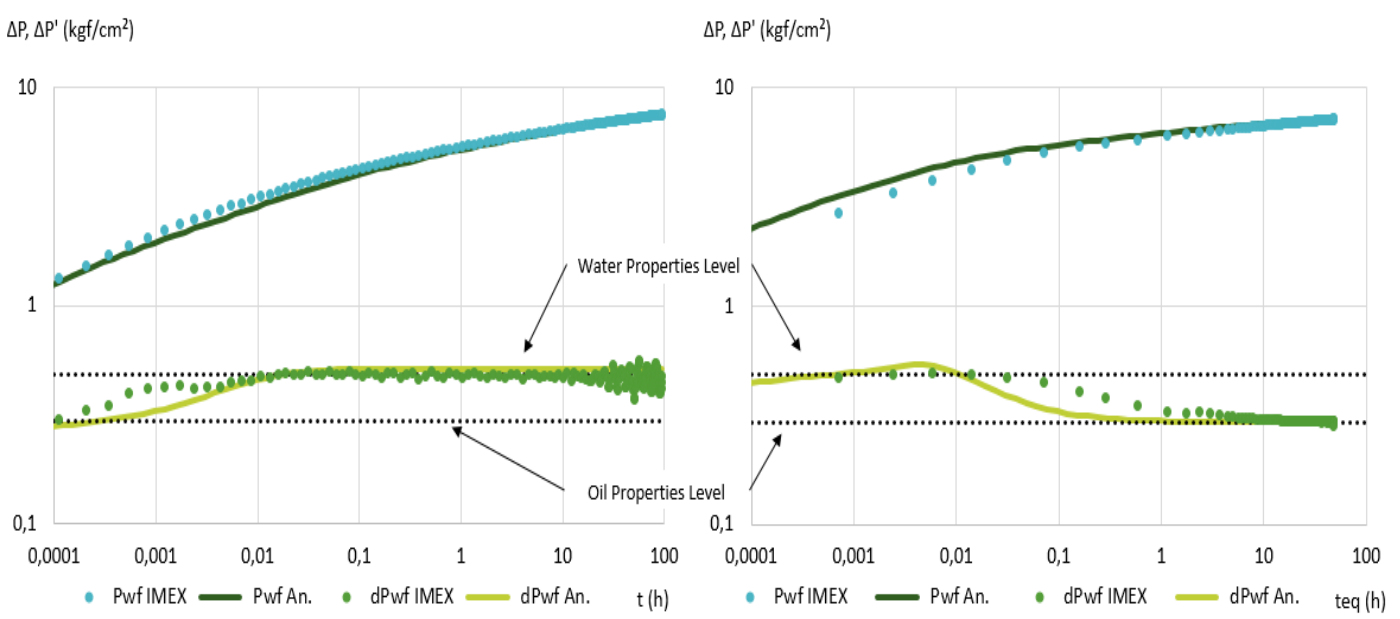

Figure 5.4: Pressure Response From Case A2 During Injection (Left) And Falloff

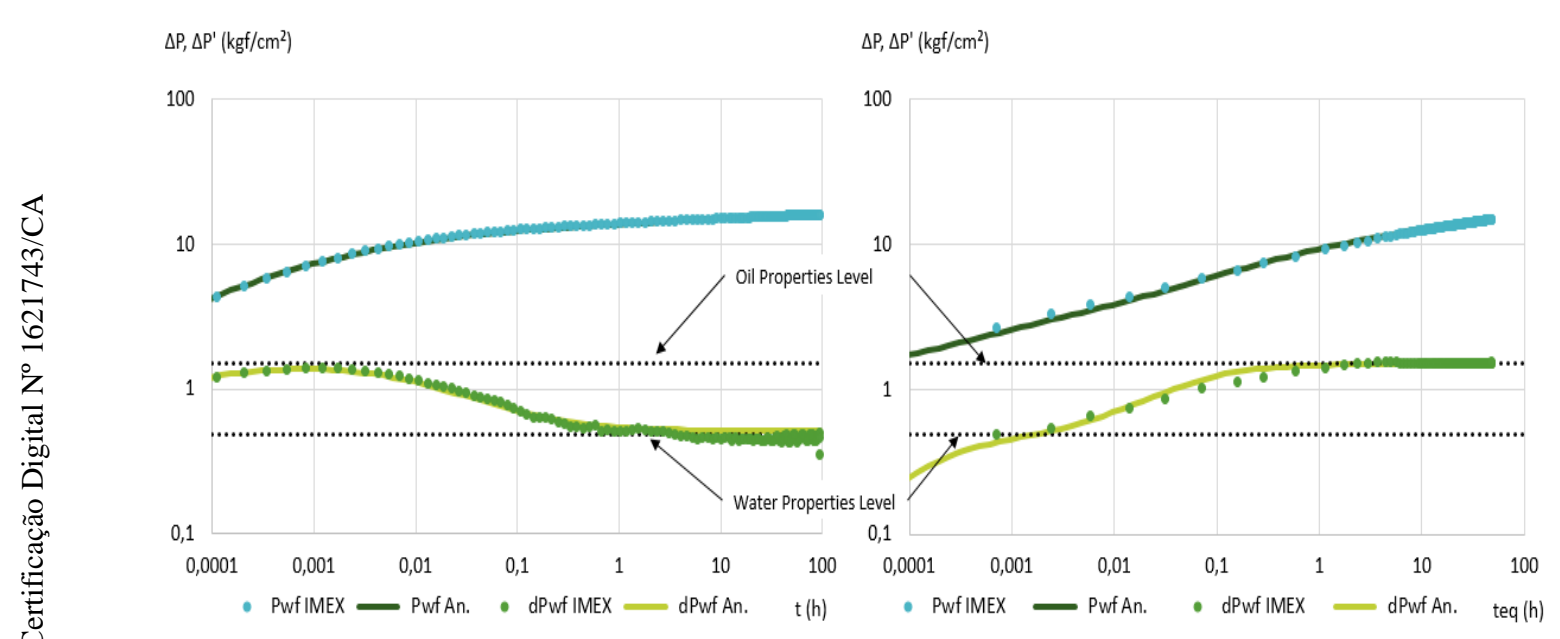

Figure 5.5: Pressure Response From Case B1 During Injection And Falloff

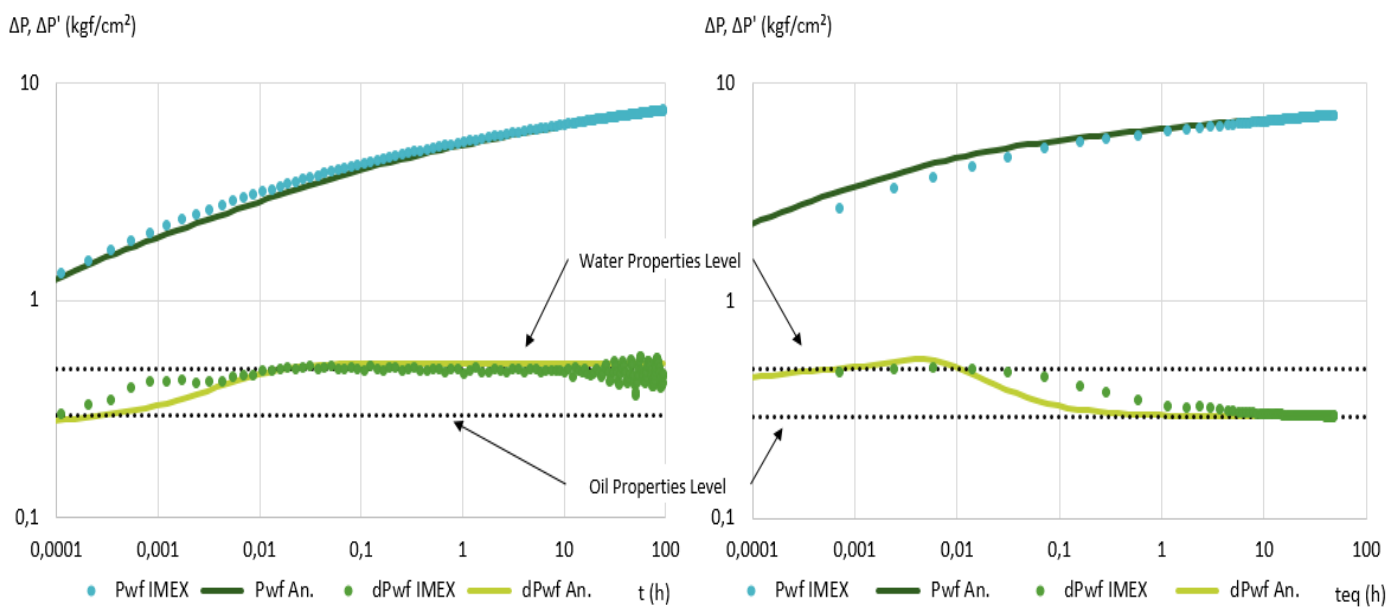

Figure 5.6: Pressure Response From Case B2 During Injection And Falloff 
Cases D1 and D2 show the pressure response in a reservoir without formation damage and with different permeabilities in each layer. Thickness is the same in every layers and the permeabilities were chosen such that the reservoir equivalent permeability is the same as the reference case. Agreement between the numerical simulator and the analytical model in cases D1 and D2 suggests that flow between layers through the wellbore is negligible, as foreseen by the hypothesis made during the development of equation (4-32).

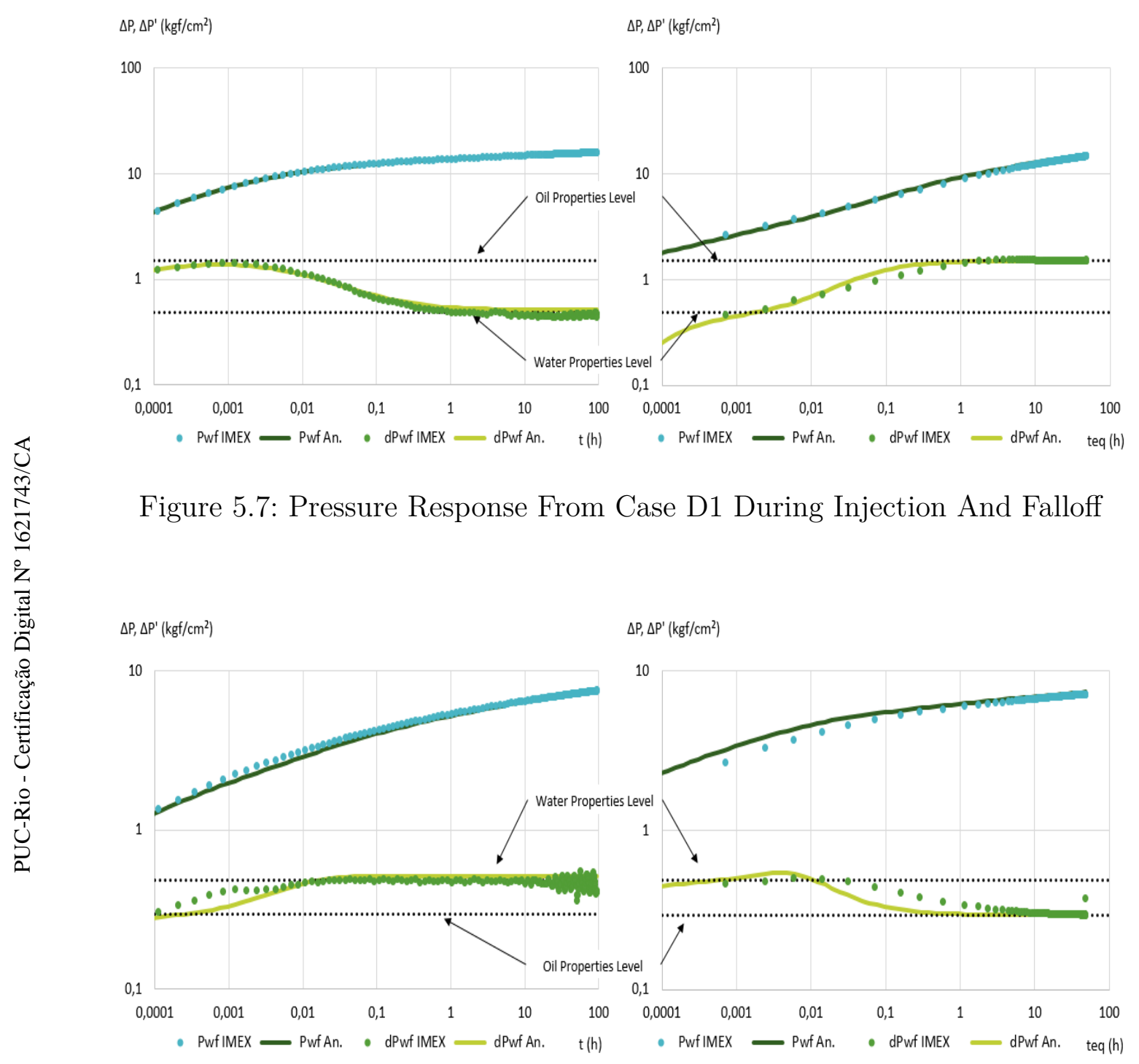

Figure 5.8: Pressure Response From Case D2 During Injection And Falloff

\section{3}

\section{Cases With Skin}

Results for cases F to Q are displayed in figures 5.9 to 5.18. Here again, the results obtained through the analytical solution was close to the numerical data, as observed in the graphs. 
For all cases, pressure derivative during flow period is characterized by an abrupt decline at the transition period, followed by a quick rise before reaching the water properties level. This is a characteristic feature of reservoirs with formation damage (23). As the injected volume gets larger than the damaged zone, waterfront transits from one region with lower permeability to another with higher permeability (21), causing the mentioned sharp drop in pressure derivative. Case J2 (fig. 5.14) is particularly interesting, because the existence of two different damaged zones is very distinguishable in the pressure derivative behavior.

Divergences between analytical solution and numerical simulated data (e.g., flow period in cases with $\hat{M}>1$ ) are related to the numerical calculation procedure. Flow simulator assumes that water is injected by a zero-radius wellbore, that is, well is considered to be a source line. Moreover, the pressure is evaluated as the average pressure in each grid block. This implies the evaluated pressure is influenced by the radial step in the simulation grid (8). Although such factors are inherent to the numerical simulation, they are not an issue for the analytical model.

Assuming the reservoir is in hydrostatic equilibrium after shut-in is a quite strong hypothesis, specially for cases whose layer permeabilities and damaged zones are very different. For these cases, during the first falloff instants, flow between layers through the wellbore may occur, due to the layer flow capacity difference $(14,10)$. Neglecting this effect is a possible explanation for the deviations between analytical and numerical values of pressure derivative at early falloff time $\left(t_{e q}<0.001 \mathrm{~h}\right)$.

With the purpose of studying the flow between layers during initial falloff time, single-phase oil flows with the same reservoir configuration depicted in table 5.1 were also run on IMEX and compared to the analytical model. Results might be seen at figures 5.19 to 5.28. For all cases, it is noticeable that numerical data derivative is not constant during early falloff times. Since this behavior was detected even in cases whose layer properties were the same, such fact was considered to be a reflection of the numerical calculation issues associated to the radial grid. This is one factor that explains deviations between the flow simulator and the analytical model.

Yet, the errors associated to the flow-rates computation are more relevant in cases with formation damage. The higher flow-rate values estimated by the source line approximation imply in slightly higher values of $\Delta P_{\lambda}$ when total flow-rate is estimated through equation (4-7). In cases with $\hat{M}>1$, the pressure term associated to the mobility differences is negative. Hence, during initial falloff times, this reflects in lower pressure derivative values. In cases 
whose flow is unfavorable to water, this error is hidden, since the $\Delta P_{\lambda}$ term is already positive. As the zero-rate pulse propagates throughout the reservoir, the hypothesis becomes valid and derivative behavior becomes similar to the cases without skin.

Cases F1 and F2 are the reference cases for reservoirs with formation damage. They show the pressure response in a single-layer reservoir and skin factor $S \neq 0$.

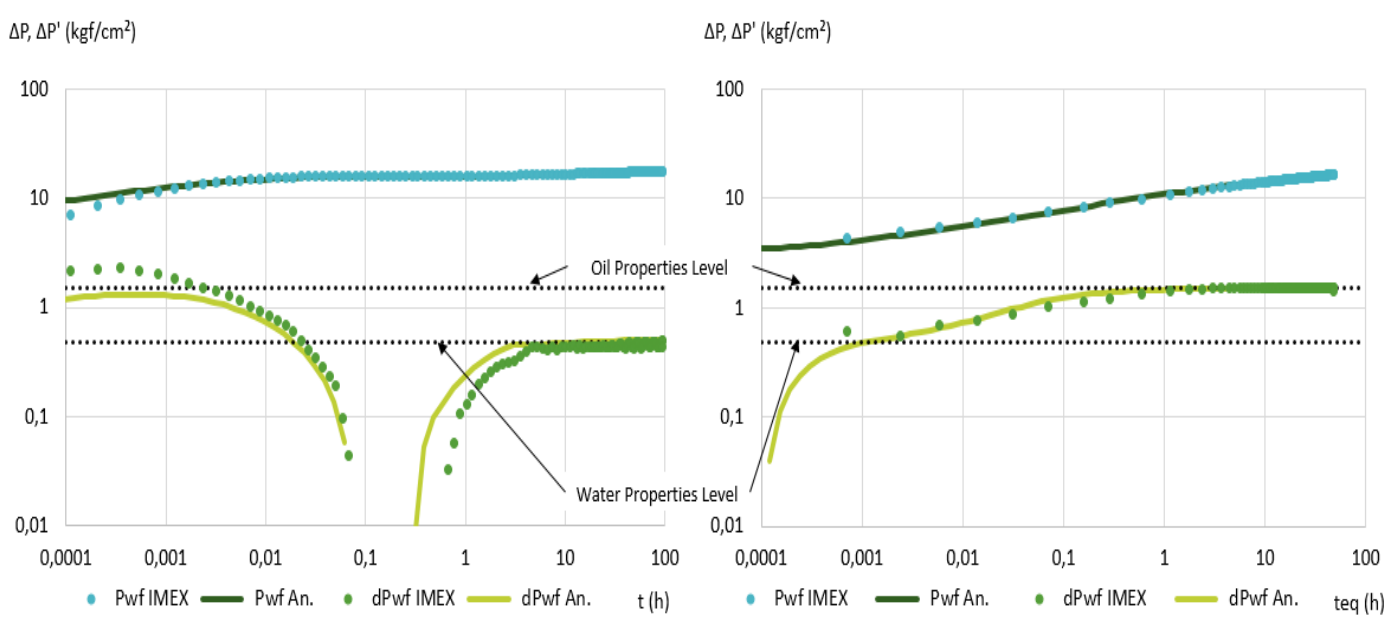

Figure 5.9: Pressure Response From Case F1 During Injection And Falloff

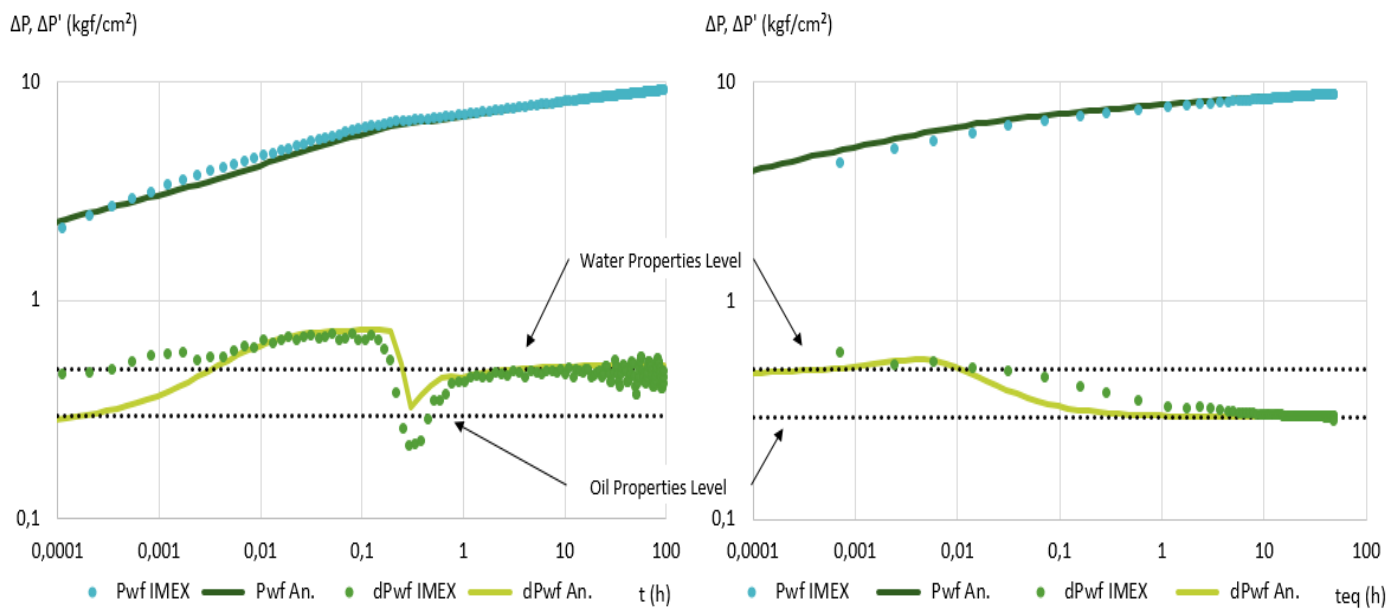

Figure 5.10: Pressure Response From Case F2 During Injection And Falloff 
Cases $\mathrm{H} 1$ and $\mathrm{H} 2$ show the pressure response in a reservoir with two equal layers, both with the same skin factor.

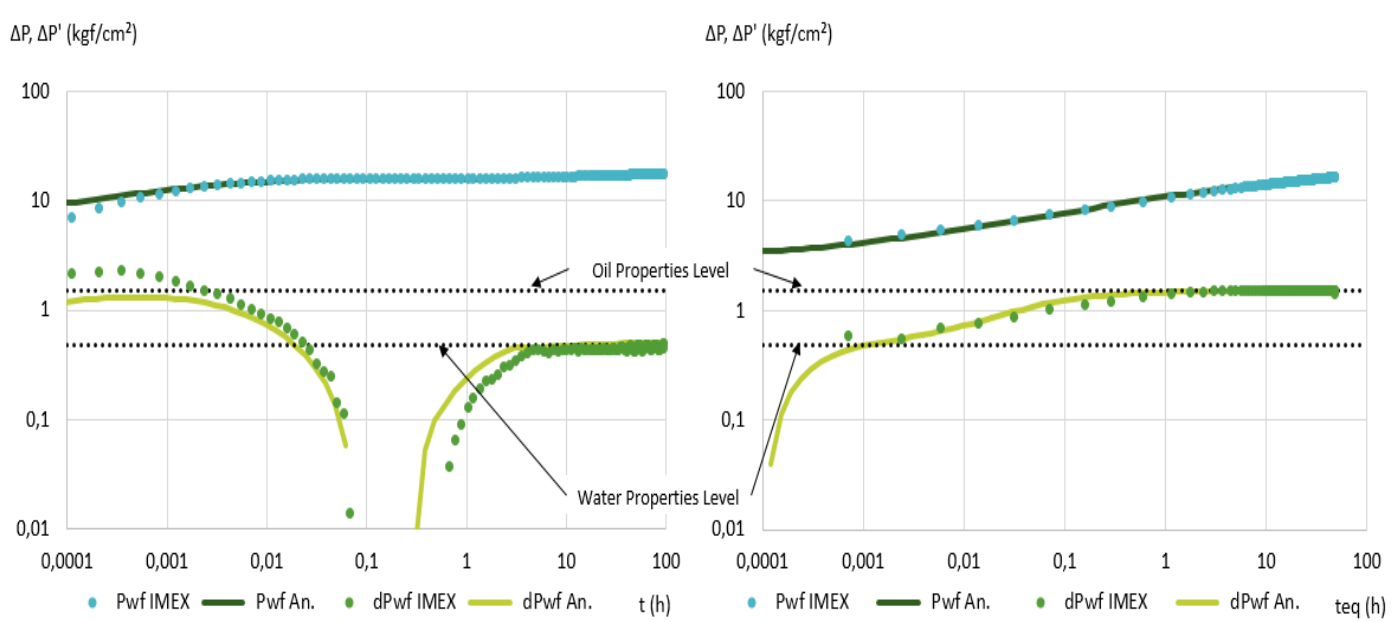

Figure 5.11: Pressure Response From Case H1 During Injection And Falloff

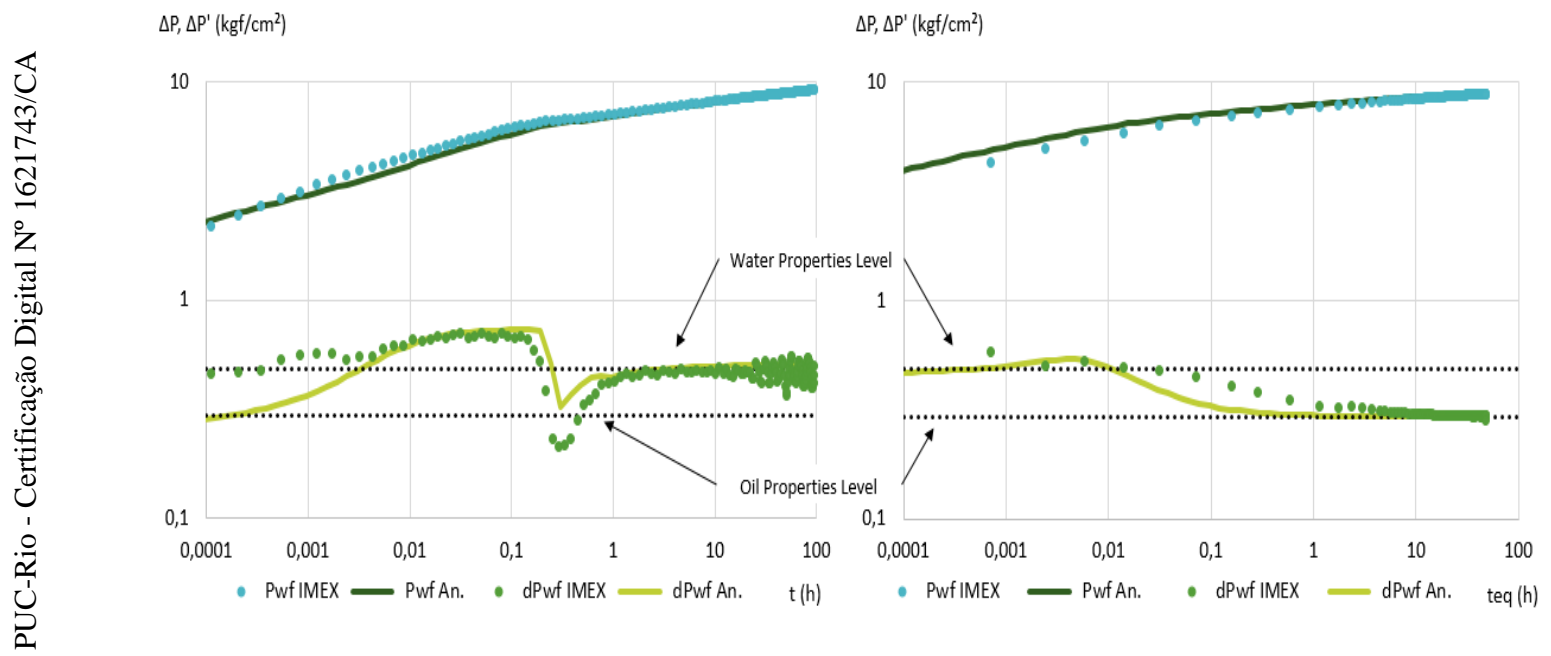

Figure 5.12: Pressure Response From Case H2 During Injection And Falloff 
The pressure behavior in a two-layer reservoir with two distinct damaged regions is displayed in cases $\mathrm{J} 1$ and $\mathrm{J} 2$. As mentioned, in case J2. the pressure derivative profile clearly points the waterfront passage through each damaged zone.

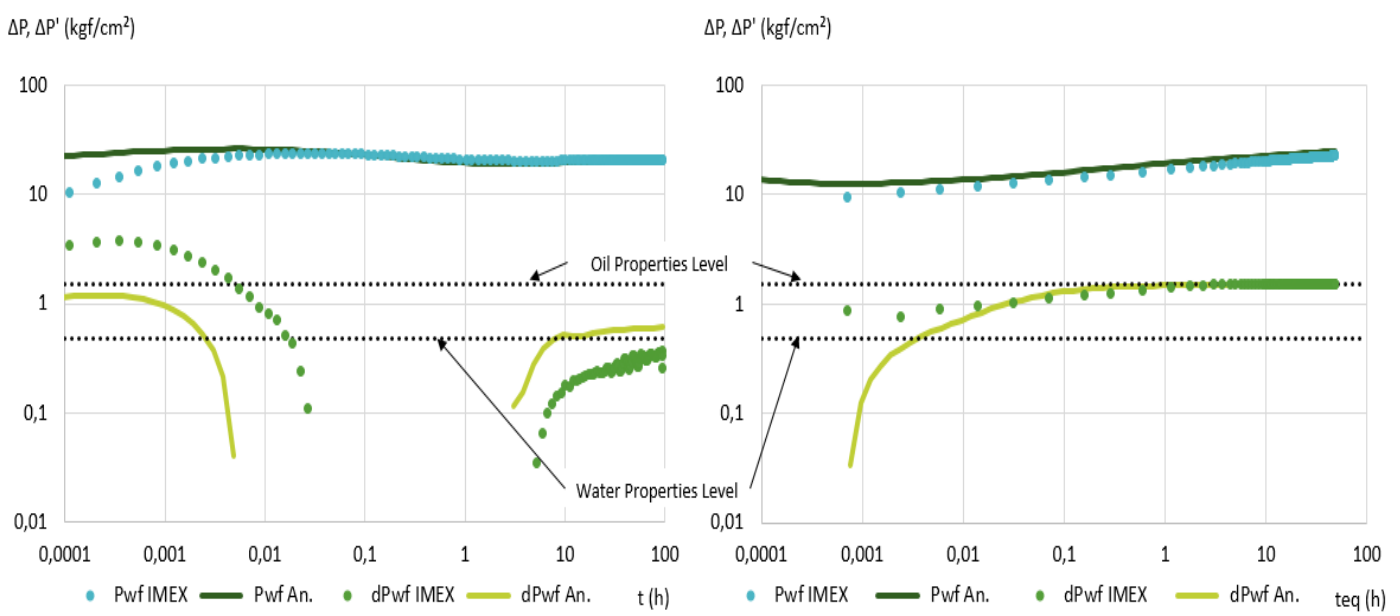

Figure 5.13: Pressure Response From Case J1 During Injection And Falloff

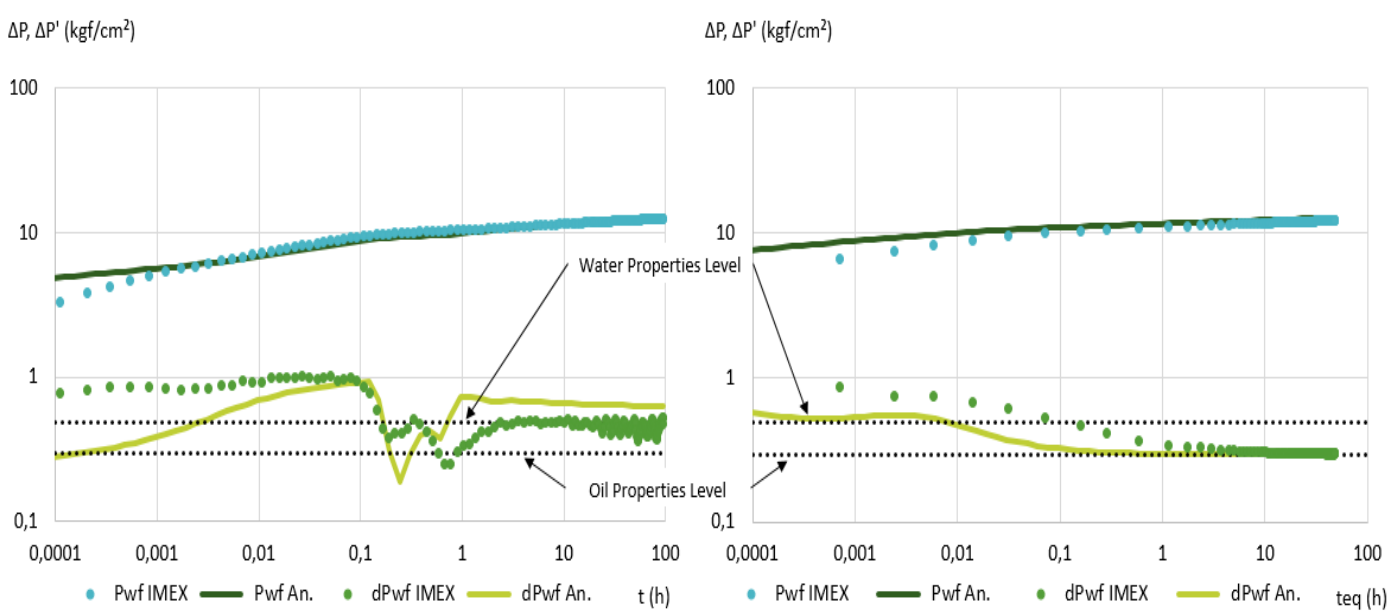

Figure 5.14: Pressure Response From Case J2 During Injection And Falloff 
Cases L1 and L2 aim to assess the analytical model in reservoirs where the skin effect is not present in every layer. They consist on a reservoir with three equal layers, but only two of them present formation damage.

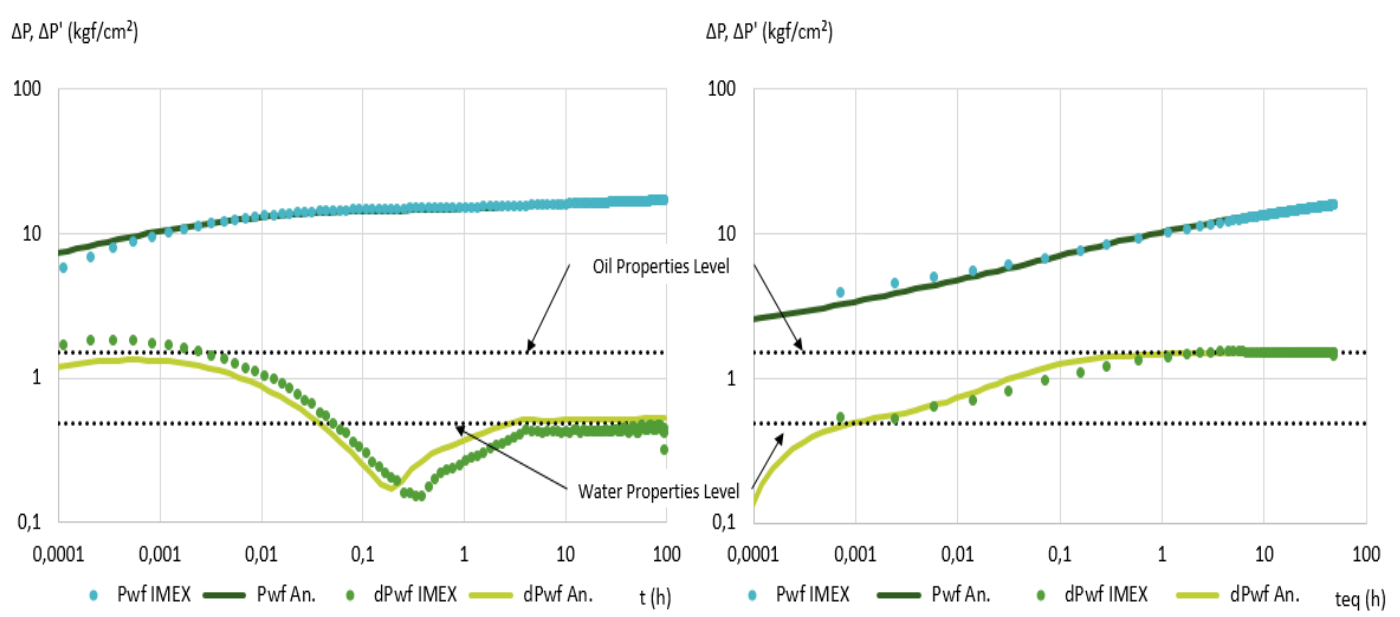

Figure 5.15: Pressure Response From Case L1 During Injection And Falloff

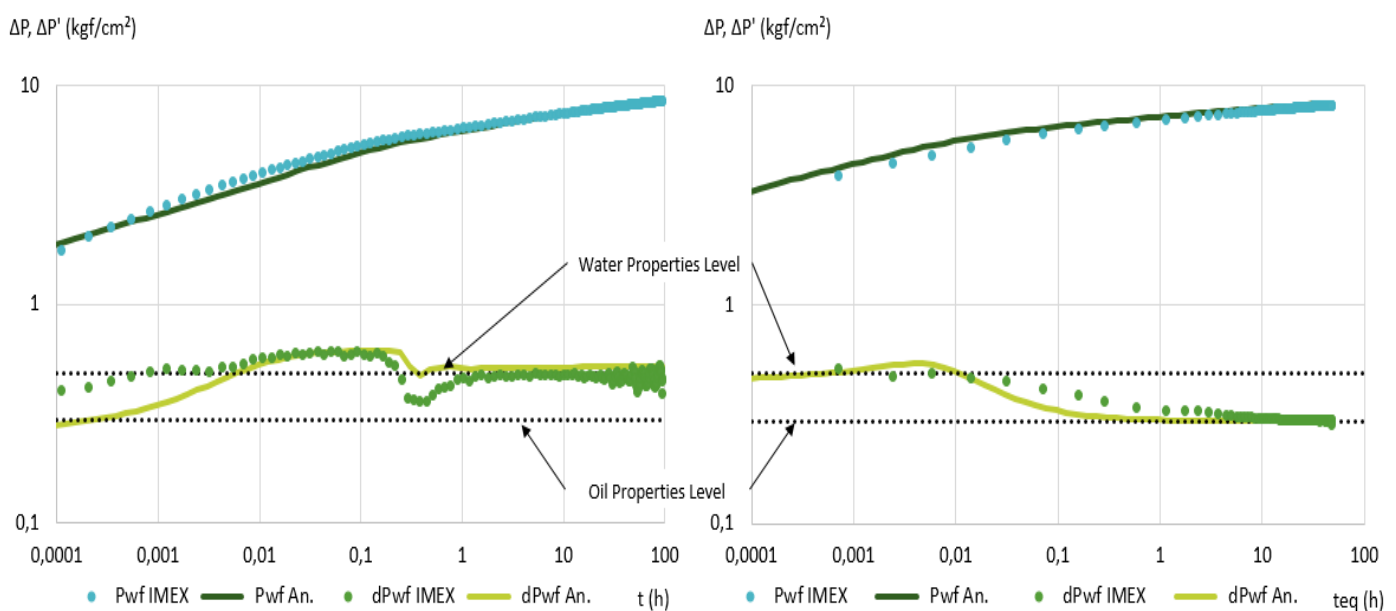

Figure 5.16: Pressure Response From Case L2 During Injection And Falloff 
Cases Q1 and Q2 are, perhaps, the two cases more related to the physical reality. Here, reservoir presents five distinct layers, all of them with skin factor $S \neq 0$. However, damaged zone properties were chosen such that skin factor would be the same in every layer. Again, the hypothesis of negligible flow between layers during falloff is tested. The agreement between analytical model and numerical simulator suggest one more time that such hypothesis is valid.

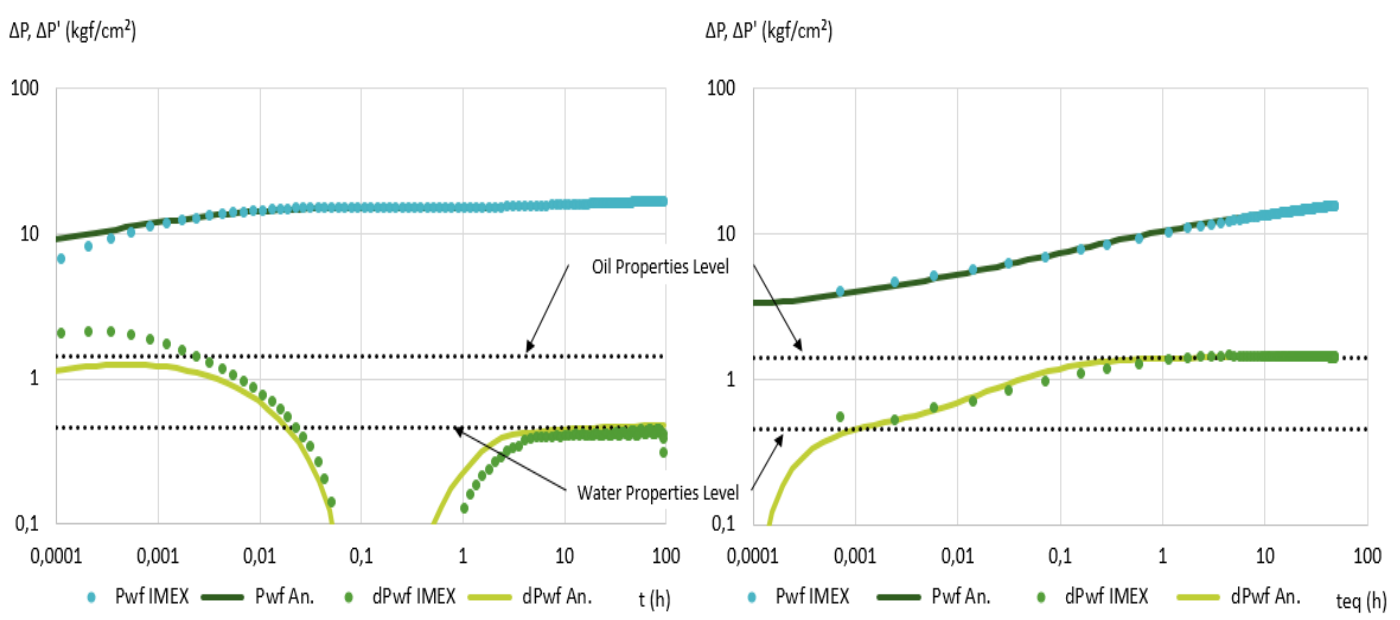

Figure 5.17: Pressure Response From Case Q1 During Injection And Falloff

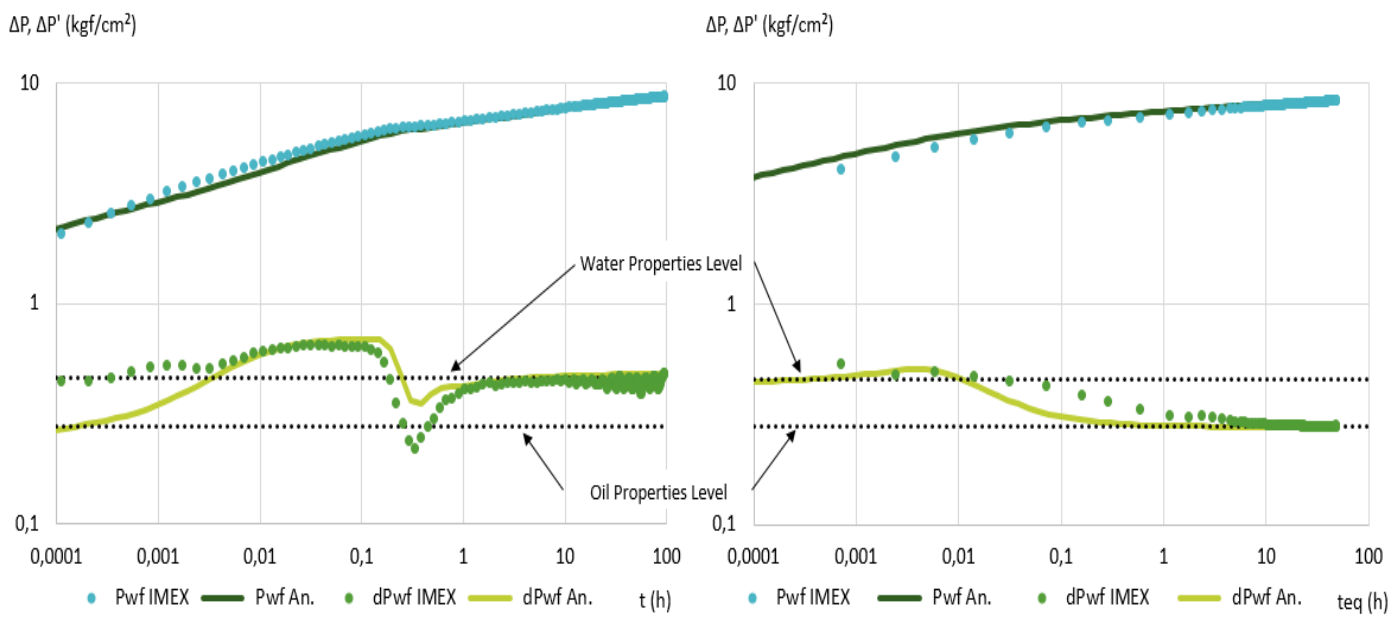

Figure 5.18: Pressure Response From Case Q2 During Injection And Falloff 
The pressure behavior for single-phase oil flow in cases F to Q are displayed below in figures 5.19 to 5.28 .
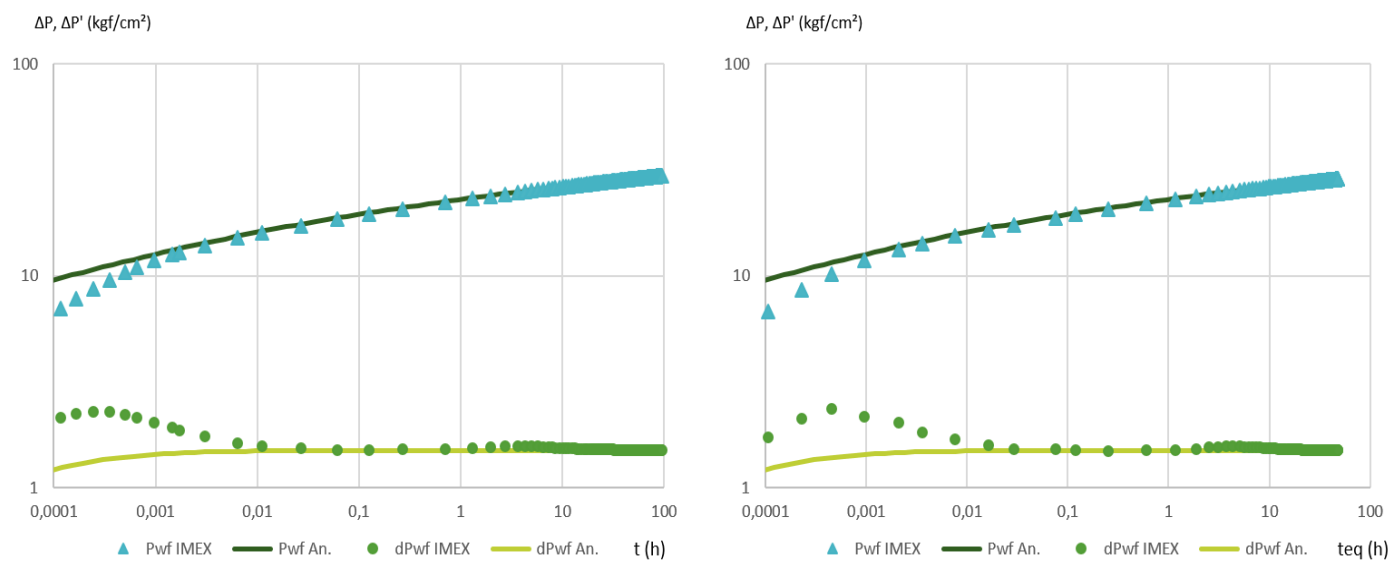

Figure 5.19: Single-Phase Oil Flow For Case F1 During Injection (Left) And Falloff
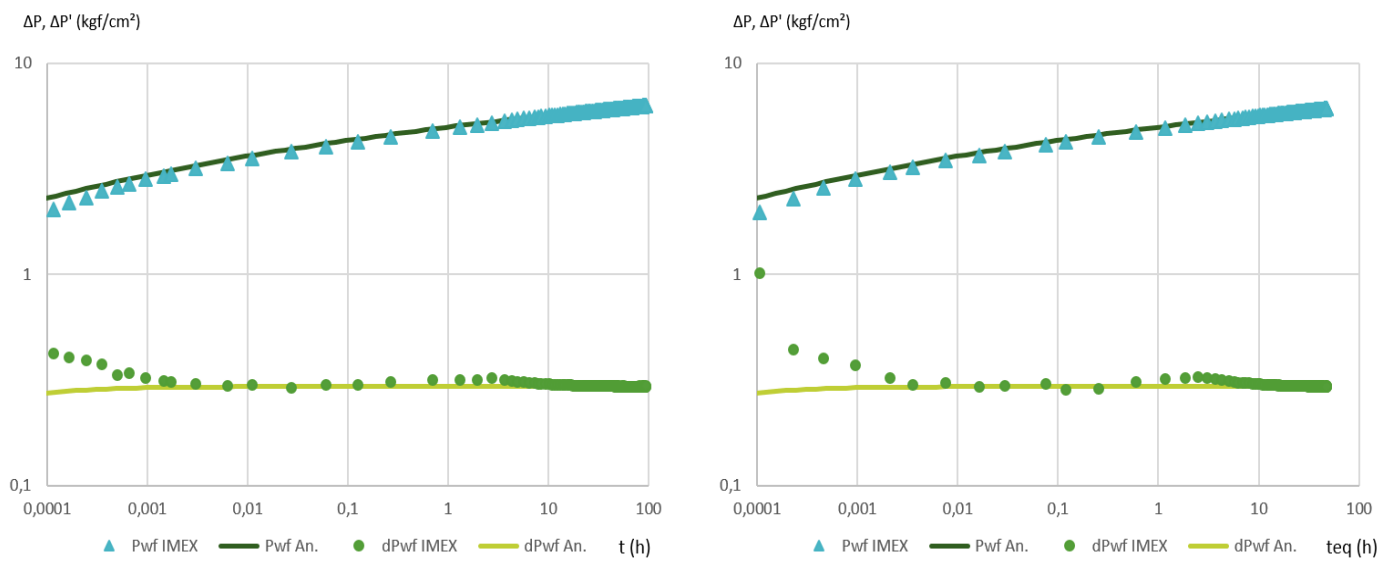

Figure 5.20: Single-Phase Oil Flow For Case F2 During Injection (Left) And Falloff 

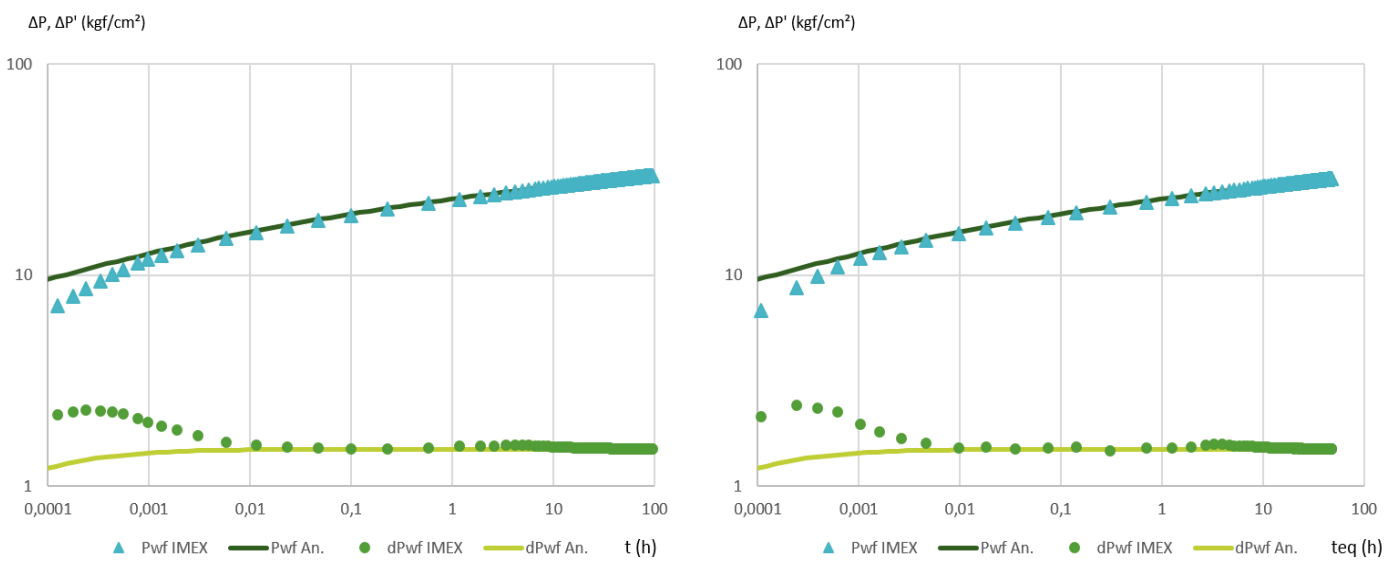

Figure 5.21: Single-Phase Oil Flow For Case H1 During Injection And Falloff
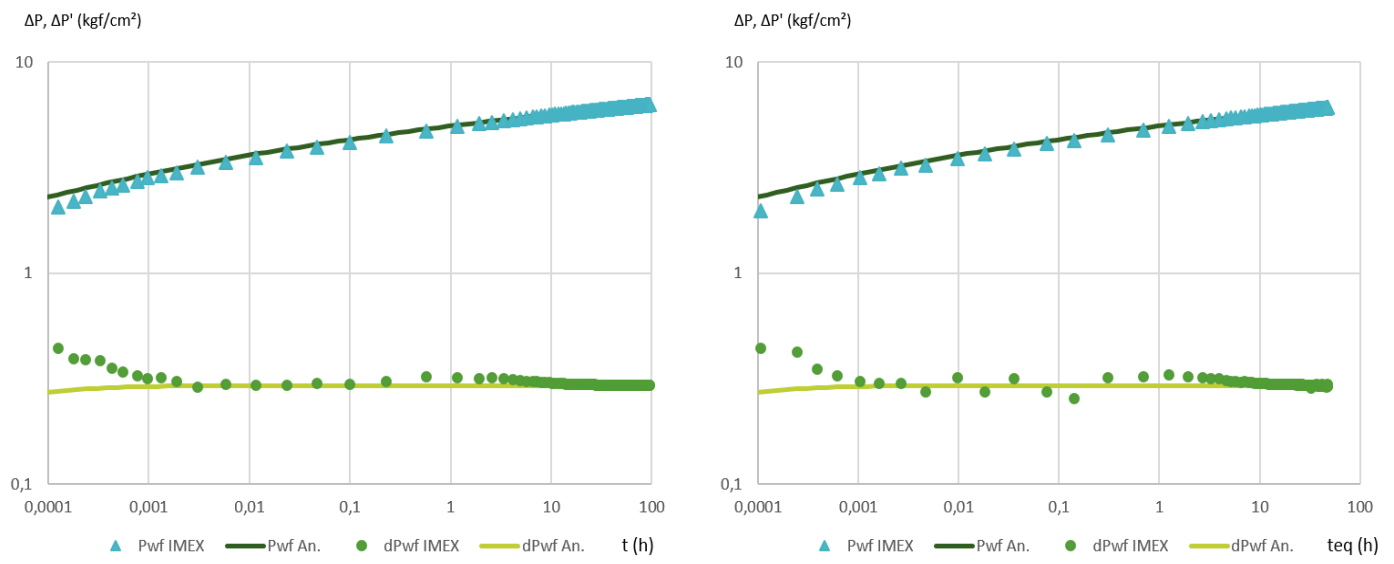

Figure 5.22: Single-Phase Oil Flow For Case H2 During Injection And Falloff
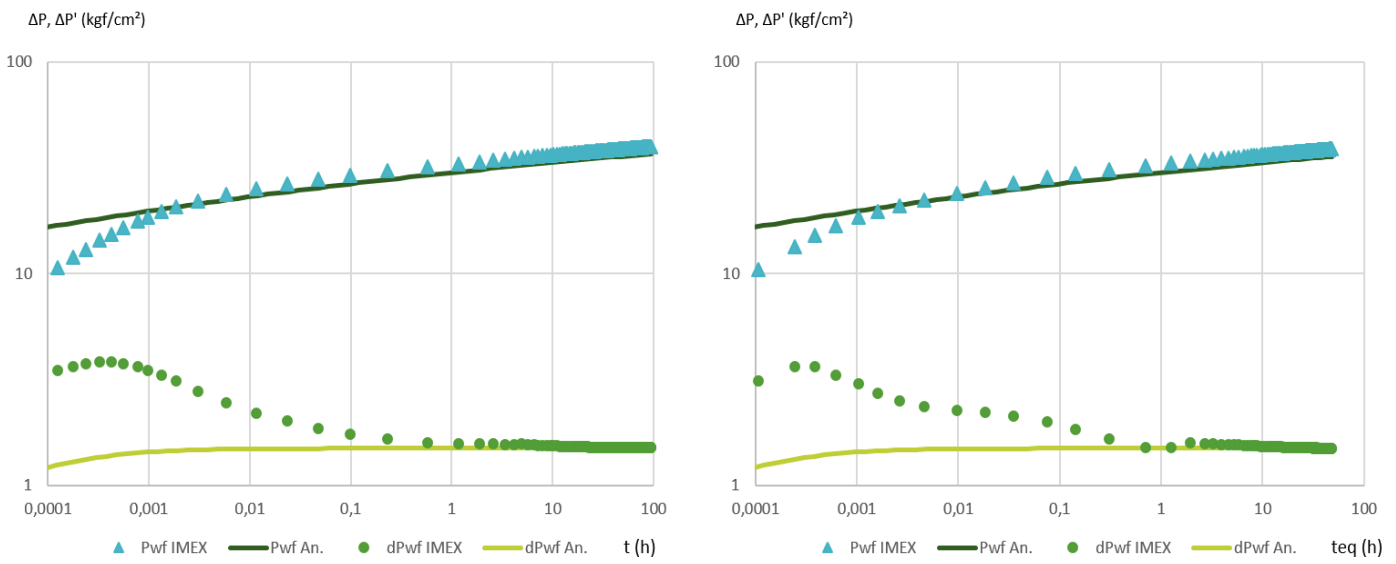

Figure 5.23: Single-Phase Oil Flow For Case J1 During Injection And Falloff 

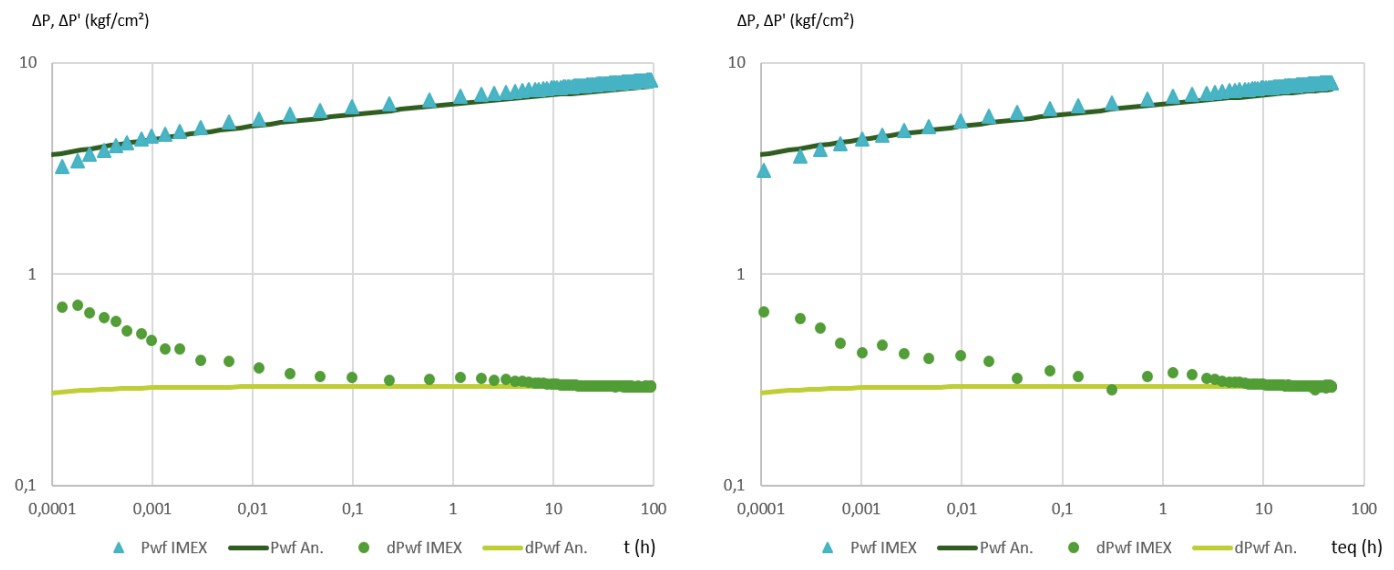

Figure 5.24: Single-Phase Oil Flow For Case J2 During Injection And Falloff
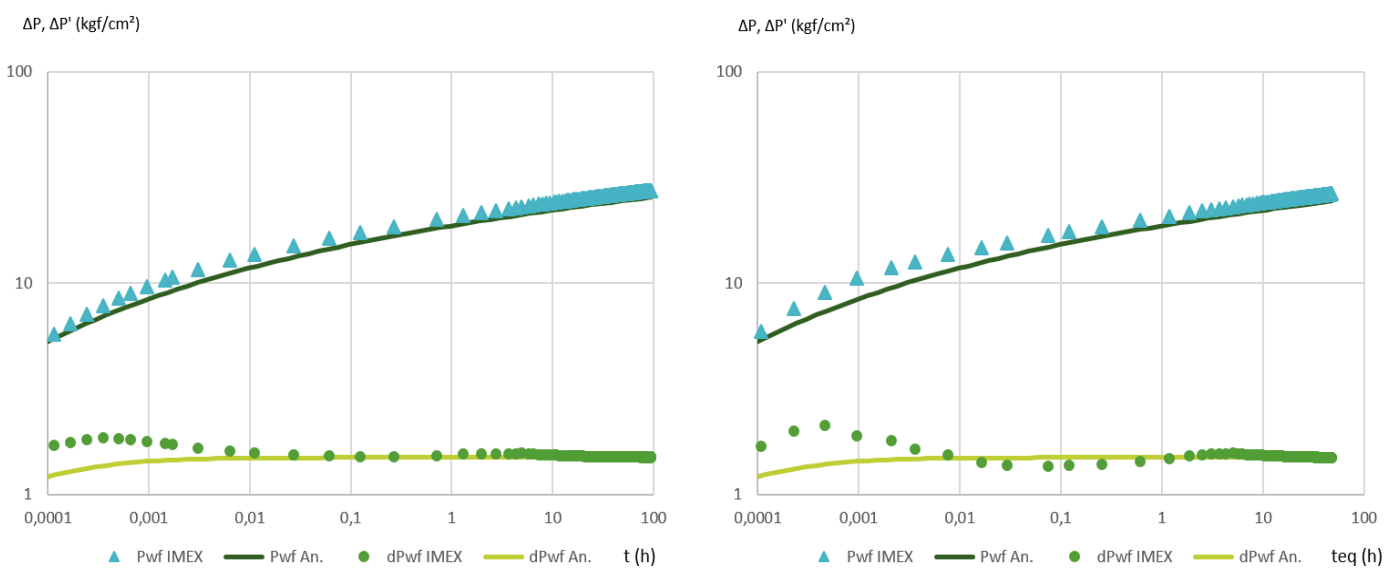

Figure 5.25: Single-Phase Oil Flow For Case L1 During Injection And Falloff
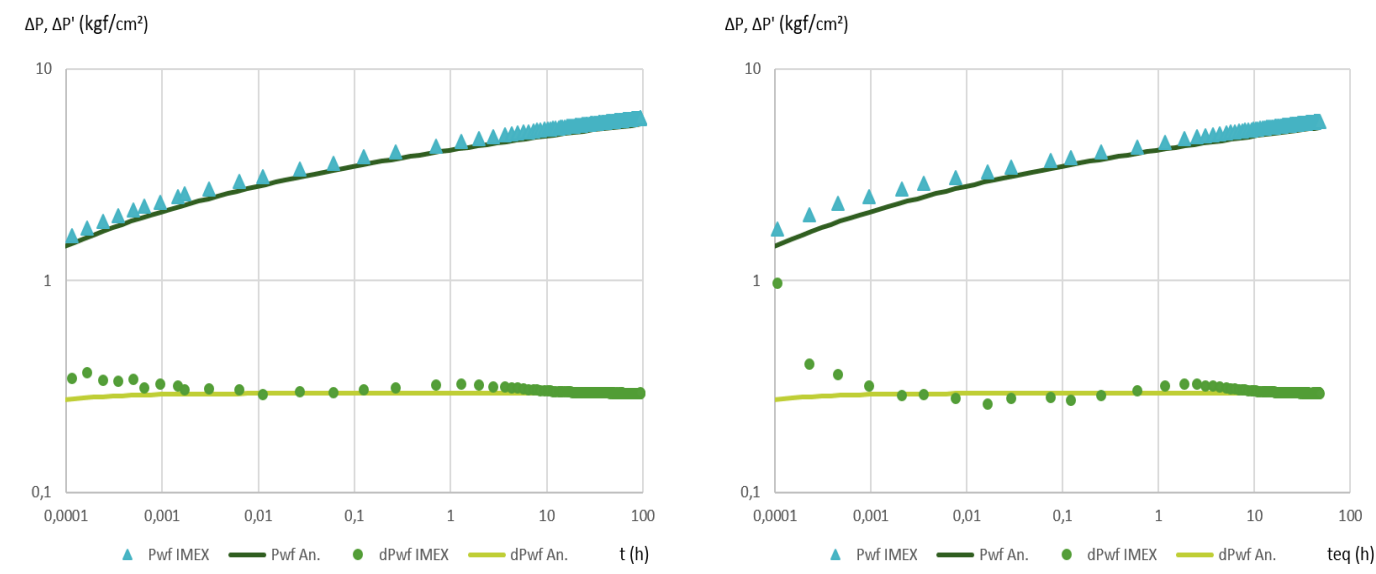

Figure 5.26: Single-Phase Oil Flow For Case L2 During Injection And Falloff 

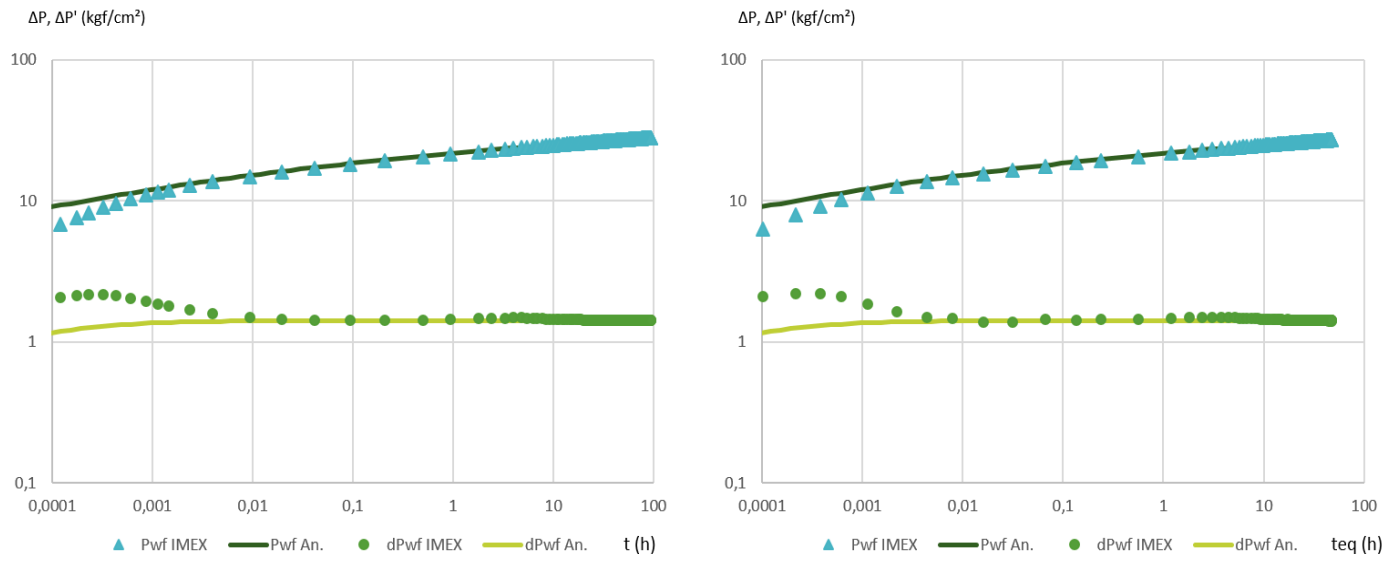

Figure 5.27: Single-Phase Oil Flow For Case Q1 During Injection And Falloff

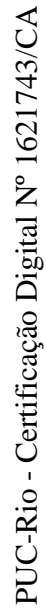
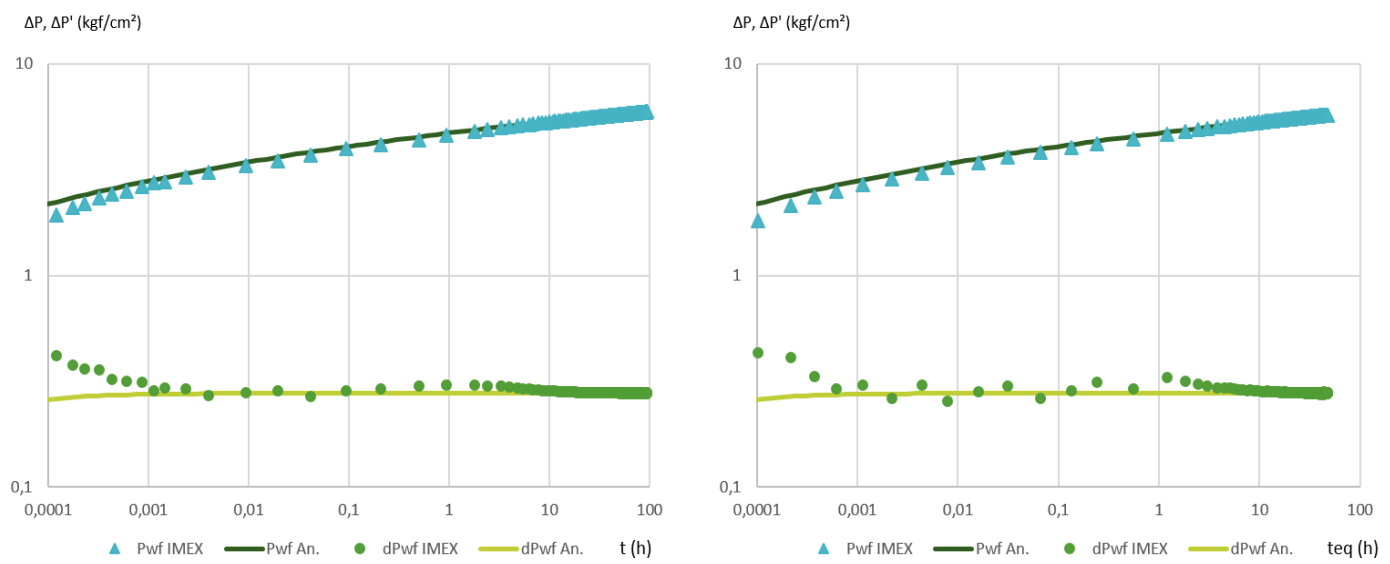

Figure 5.28: Single-Phase Oil Flow For Case Q2 During Injection And Falloff 


\section{4 \\ Determining The Reservoir Equivalent Permeability}

As depicted in sections 5.2 and 5.3, the pressure derivative profile contains two flatten regions seen, one defined by the oil mobility and another related to water properties. Thus, two straight lines are observed in the semilog pressure vs. time graph (?). The pressure term associated to the mobility differences might be understood as an apparent skin factor. Hence, the reservoir equivalent permeability may be estimated using the following equation, derived from the single-phase formulation:

$$
k_{e q}=\frac{1.151 \alpha_{p} q_{i n j}}{\bar{h} \hat{\lambda}_{f} m_{f}},
$$

where $\alpha_{p}$ is the unit conversion constant. For a consistent set of units, $\alpha_{p}=1$, whereas for the set of Brazilian field units, $\alpha_{p}=19.03$. The straight line angular coefficient is denoted by $m_{f}$, where the subscript $f$ indicates the phase associated to the straight line considered.

Therefore, falloff data were used to generate semilog graphs. Pressure data were plotted against Horner time, defined as:

$$
t_{H}=\frac{t_{p}+\Delta t}{\Delta t}
$$

Semilog straight lines and their slopes were determined through the trend line drawer available on Excel. Results for numerical and analytical data are displayed in tables 5.2 and 5.3, respectively. Figures 5.29 to 5.32 show the semilog graph for cases F1. F2. J1 and J2.

Equivalent permeability calculated according to equation (5-4) using semilog graphs were very close to the input values for all cases, both for water and oil properties, expect case J1. Due to the same causes discussed in sections 5.2 and 5.3, analytical model is subject to noticeable error during early falloff times (that means, higher Horner times). Hence, the formation of the first semilog straight line may be impaired. Case J1 was more sensitive to those factors, even though they were present in all cases. However, semilog line relative to oil properties is well formed for all cases, and resulted in an estimated equivalent permeability with error of about $1 \%$.

Equivalent permeabilites calculated using numerical data implied in excellent results when oil mobility was used. On the other hand, water properties resulted in significant error, which may be explained by the flow between layers, impairing the formation of the first semilog straight line.

It is interesting to observe that, in field tests, early falloff data usually present too much noise. Thus, water semilog straight line is not detectable and the reservoir equivalent permeability is estimated using the straight line 
relative to oil properties in a typical field test. That means both numerical and analytical data allowed a quite accurate estimate for the equivalent permeability when the most relevant straight line (the one associated to oil mobility) is used.
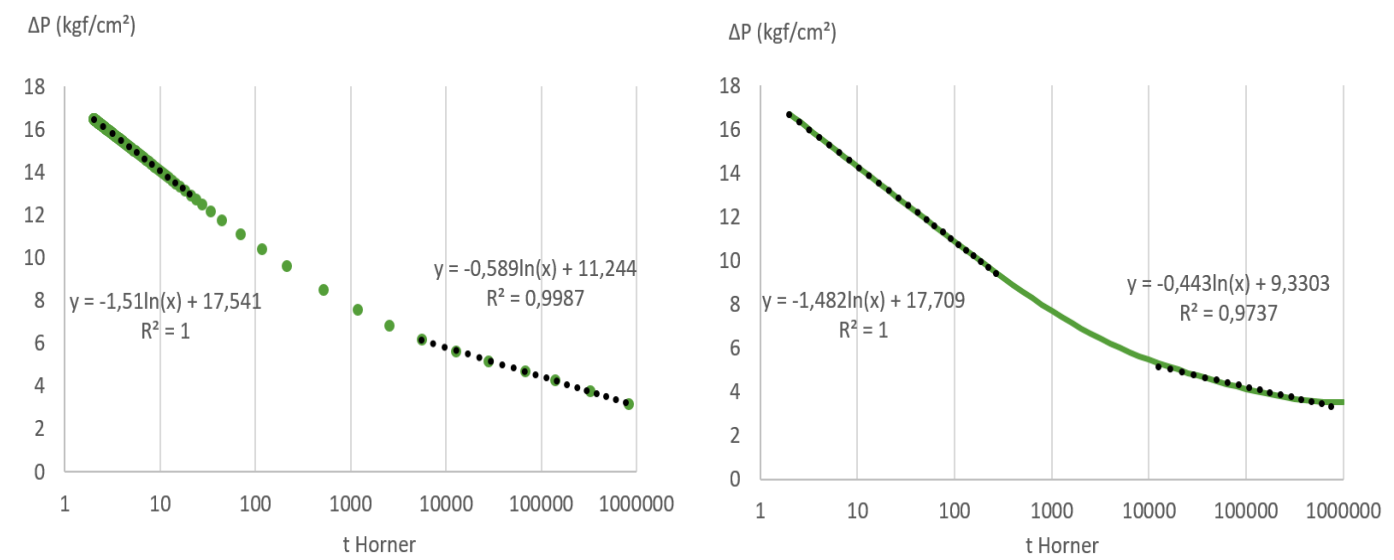

Figure 5.29: Semilog Graph For Case F1 - Numerical Data On The Left And Analytical Data On The Right
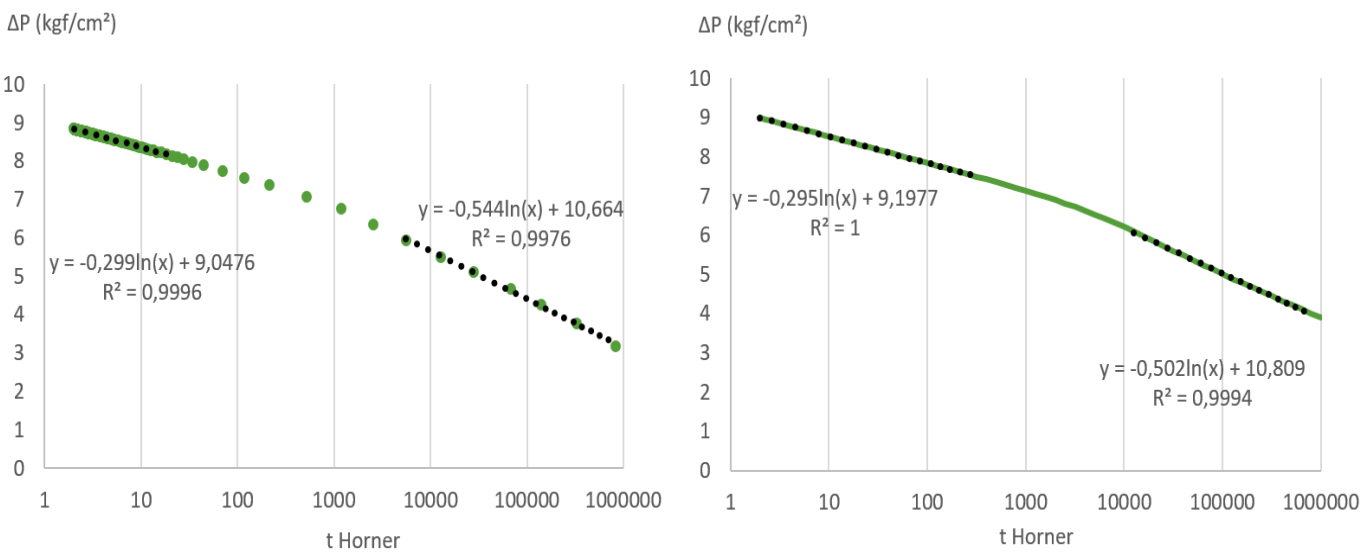

Figure 5.30: Semilog Graph For Case F2 - Numerical Data On The Left And Analytical Data On The Right 

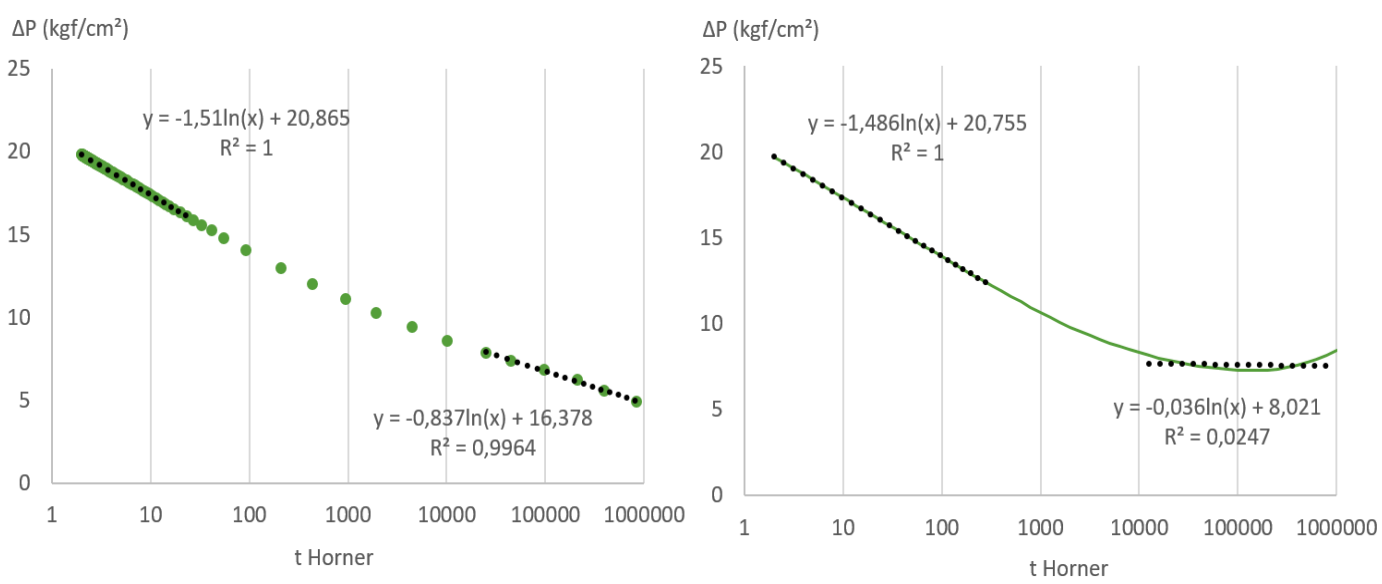

Figure 5.31: Semilog Graph For Case J1 - Numerical Data On The Left And Analytical Data On The Right

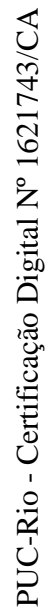
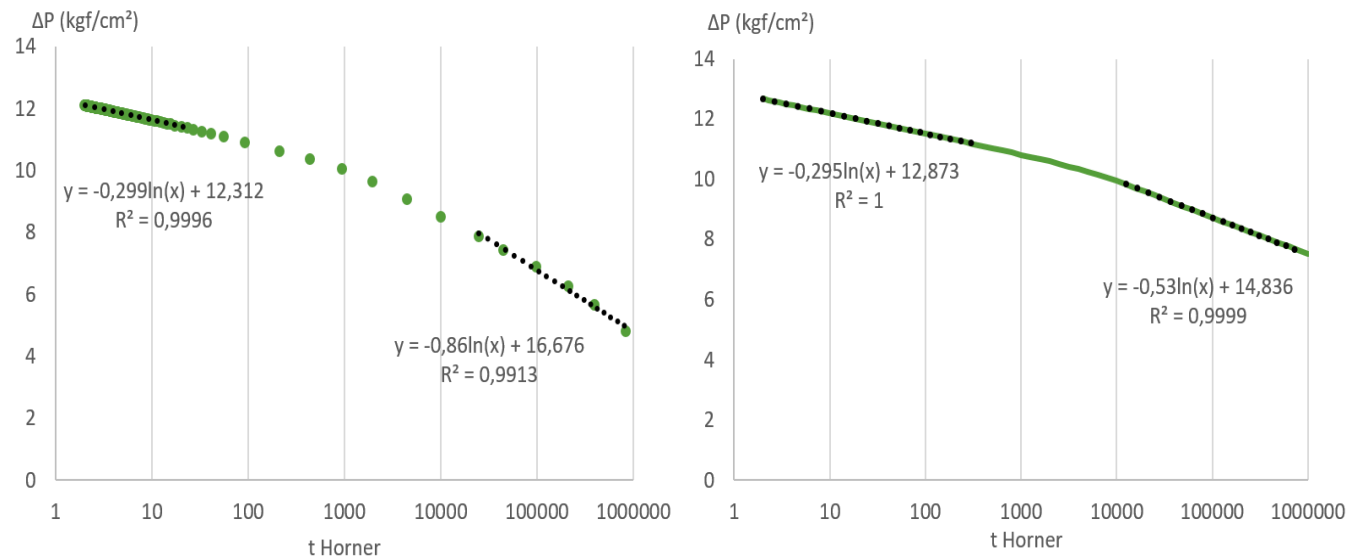

Figure 5.32: Semilog Graph For Case J2 - Numerical Data On The Left And Analytical Data On The Right 
Table 5.2: Equivalent Permeabilities Calculated Using IMEX Data

\begin{tabular}{|c|c|c|c|c|c|}
\hline Case & $\mathrm{k}_{\mathrm{eq}}(\mathrm{mD})$ & Phase & $\mathrm{m}_{\mathrm{f}} \mathrm{Num}$. & $\mathrm{k}_{\mathrm{eq}}$ Num. $(\mathrm{mD})$ & Error $(\%)$ \\
\hline \multirow{2}{*}{ A1 } & \multirow{2}{*}{1000} & Oil & 1.508 & 993 & "-0.7 \\
\hline & & Water & 0.641 & 757 & -24.3 \\
\hline \multirow{2}{*}{$\mathrm{A} 2$} & \multirow{2}{*}{1000} & Oil & 0.298 & 985 & -1.5 \\
\hline & & Water & 0.498 & 974 & -2.6 \\
\hline \multirow{2}{*}{ B1 } & \multirow{2}{*}{1000} & Oil & 1.509 & 992 & -0.8 \\
\hline & & Water & 0.641 & 757 & -24.3 \\
\hline \multirow{2}{*}{ B2 } & \multirow{2}{*}{1000} & Oil & 0.298 & 985 & -1.5 \\
\hline & & Water & 0.497 & 976 & -2.4 \\
\hline \multirow{2}{*}{ D1 } & \multirow{2}{*}{1000} & Oil & 1.512 & 990 & -1.0 \\
\hline & & Water & 0.605 & 802 & -19.8 \\
\hline \multirow{2}{*}{ D2 } & \multirow{2}{*}{1000} & Oil & 0.298 & 985 & -1.5 \\
\hline & & Water & 0.498 & 974 & -2.6 \\
\hline \multirow{2}{*}{ F1 } & \multirow{2}{*}{1000} & Oil & 1.510 & 992 & -0.8 \\
\hline & & Water & 0.589 & 823 & -17.7 \\
\hline \multirow{2}{*}{$\mathrm{F} 2$} & \multirow{2}{*}{1000} & Oil & 0.299 & 982 & -1.8 \\
\hline & & Water & 0.543 & 893 & -10.7 \\
\hline \multirow{2}{*}{ H1 } & \multirow{2}{*}{1000} & Oil & 1.511 & 991 & -0.9 \\
\hline & & Water & 0.577 & 840 & -16.0 \\
\hline \multirow{2}{*}{$\mathrm{H} 2$} & \multirow{2}{*}{1000} & Oil & 0.299 & 982 & -1.8 \\
\hline & & Water & 0.558 & 869 & -13.1 \\
\hline \multirow{2}{*}{ J1 } & \multirow{2}{*}{1000} & Oil & 1.510 & 992 & -0.8 \\
\hline & & Water & 0.837 & 579 & -42.1 \\
\hline \multirow{2}{*}{$\mathrm{J} 2$} & \multirow{2}{*}{1000} & Oil & 0.299 & 982 & -1.8 \\
\hline & & Water & 0.859 & 565 & -43.5 \\
\hline \multirow{2}{*}{ L1 } & \multirow{2}{*}{1000} & Oil & 1.510 & 992 & -0.8 \\
\hline & & Water & 0.551 & 880 & -12.0 \\
\hline \multirow{2}{*}{ L2 } & \multirow{2}{*}{1000} & Oil & 0.299 & 982 & -1.8 \\
\hline & & Water & 0.501 & 968 & -3.2 \\
\hline \multirow{2}{*}{ Q1 } & \multirow{2}{*}{1060} & Oil & 1.507 & 994 & -0.6 \\
\hline & & Water & 0.543 & 893 & -15.7 \\
\hline Q? & 1060 & Oil & 0.282 & 1041 & -1.8 \\
\hline & 1060 & Water & 0.540 & 898 & -15.3 \\
\hline
\end{tabular}


Table 5.3: Equivalent Permeabilities Computed Using Analytical Data

\begin{tabular}{|c|c|c|c|c|c|}
\hline Case & $\mathrm{k}_{\mathrm{eq}}(\mathrm{mD})$ & Phase & $m_{\mathrm{f}} \mathrm{An}$. & $\mathrm{k}_{\mathrm{eq}}$ An. (mD) & Error $(\%)$ \\
\hline \multirow{2}{*}{ A1 } & \multirow{2}{*}{1000} & Oil & 1.482 & 1010 & 1.0 \\
\hline & & Water & 0.460 & 1054 & 5.4 \\
\hline \multirow{2}{*}{$\mathrm{A} 2$} & \multirow{2}{*}{1000} & Oil & 0.295 & 995 & -0.5 \\
\hline & & Water & 0.500 & 970 & -3.0 \\
\hline \multirow{2}{*}{ B1 } & \multirow{2}{*}{1000} & Oil & 1.482 & 1010 & 1.0 \\
\hline & & Water & 0.460 & 1054 & 5.4 \\
\hline \multirow{2}{*}{ B2 } & \multirow{2}{*}{1000} & Oil & 0.295 & 995 & -0.5 \\
\hline & & Water & 0.500 & 970 & -3.0 \\
\hline \multirow{2}{*}{ D1 } & \multirow{2}{*}{1000} & Oil & 1.483 & 1010 & 1.0 \\
\hline & & Water & 0.458 & 1059 & 5.9 \\
\hline \multirow{2}{*}{ D2 } & \multirow{2}{*}{1000} & Oil & 0.295 & 995 & -0.5 \\
\hline & & Water & 0.504 & 962 & -3.8 \\
\hline \multirow{2}{*}{ F1 } & \multirow{2}{*}{1000} & Oil & 1.482 & 1010 & 1.0 \\
\hline & & Water & 0.443 & 1095 & 9.5 \\
\hline \multirow{2}{*}{$\mathrm{F} 2$} & \multirow{2}{*}{1000} & Oil & 0.295 & 995 & -0.5 \\
\hline & & Water & 0.502 & 966 & -3.4 \\
\hline \multirow{2}{*}{ H1 } & \multirow{2}{*}{1000} & Oil & 1.482 & 1010 & 1.0 \\
\hline & & Water & 0.443 & 1095 & 9.5 \\
\hline \multirow{2}{*}{$\mathrm{H} 2$} & \multirow{2}{*}{1000} & Oil & 0.295 & 995 & -0.5 \\
\hline & & Water & 0.502 & 966 & -3.4 \\
\hline \multirow{2}{*}{ J1 } & \multirow{2}{*}{1000} & Oil & 1.486 & 1008 & 0.8 \\
\hline & & Water & 0.036 & 13471 & 1247.1 \\
\hline \multirow{2}{*}{$\mathrm{J} 2$} & \multirow{2}{*}{1000} & Oil & 0.295 & 995 & -0.5 \\
\hline & & Water & 0.530 & 915 & -8.5 \\
\hline \multirow{2}{*}{ L1 } & \multirow{2}{*}{1000} & Oil & 1.483 & 1010 & 1.0 \\
\hline & & Water & 0.477 & 1017 & 1.7 \\
\hline \multirow{2}{*}{ L2 } & \multirow{2}{*}{1000} & Oil & 0.295 & 995 & -0.5 \\
\hline & & Water & 0.501 & 968 & -3.2 \\
\hline \multirow{2}{*}{ Q1 } & \multirow{2}{*}{1060} & Oil & 1.399 & 1070 & 1.0 \\
\hline & & Water & 0.425 & 1141 & 7.6 \\
\hline O? & 1060 & Oil & 0.278 & 1056 & -0.4 \\
\hline Q2 & 1000 & Water & 0.475 & 1021 & -3.7 \\
\hline
\end{tabular}




\section{6}

\section{Conclusions And Suggestions For Future Works}

The analytical solution for the injection period in multilayer reservoirs was implemented as suggested by Barreto et al. (25). Their model reduces to the formulation proposed by Banerjee et al. (20) and Peres et al. (22) when applied to single-layer reservoirs. Pressure behavior during falloff in single-layer reservoirs was computed according to the solution developed by Peres et al. (24). Based on their formulation for falloff period in single-layer reservoirs, an expression for multilayer reservoirs was developed.

The suggested model was applied on a series of cases, with distinct number of layers, layer permeabilities, formation damage permeabilities and radii. For all cases, comparison between analytical solution and numerical simulation showed a close agreement.

The reservoir equivalent permeability estimated using falloff data and oil properties was very close to the real value for all simulated cases.

Such results verified that the proposed formulation was accurate to describe falloff pressure behavior in multilayer systems.

The analytical model developed may be used as basis for further works. One natural step is to evaluate the accuracy of the suggested falloff formulation in multilayer reservoirs with different outer boundary conditions. The study of multilayer reservoirs with more than one injector wellbore would also benefit from this work.

An extension of the analytical model to horizontal wells would attract significant industrial interest. Two other reservoir management applications consist on using the proposed formulation for injection tests with non-constant injection flow-rate and attempting to estimate individual layer skin factor through the analytical model presented. Finally, the analytical model suggested may be helpful in the study of reservoirs with crossflow. 


\section{Bibliography}

[1] BUCKLEY, S. E.; LEVERETT, M. C., Mechanism of Fluid Displacement in Sands, Petroleum Technology, May 1941, p. 107-116

[2] VAN EVERDINGEN, A. F.; HURST, W. The Application of the Laplace Transformation to Flow Problems in Reservoirs, Petroleum Transactions, December 1949 , p. 305-324

[3] HAWKINS, M. F, A Note on the Skin Effect, Journal of Petroleum Technology. December, 1956, p. 65-66

[4] LEFKOVITS, H. C.; HAZEBROEK, P.; ALLEN, E. E.; MATTHEWS, C. S., A Study of the Behavior of Bounded Reservoirs Composed of Stratified Layers, SPE Journal. March, 1961, p. 43-58

[5] COBB, W. M.; RAMEY JR., H. J.; MILLER, F. G., Well-Test Analysis for Wells Producing Commingled Zones, Journal of Petroleum Technology. January, 1972, p. 27-37

[6] ABRAMOWITZ, M.; STEGUN, I. A., Handbook of Mathematical Functions with Formulas, Graphs and Mathematical Tables, 10h printing. CIDADE, 1972

[7] RAGHAVAN, R.; T OPALOGLU, H. N.; COBB, W. M.; RAMEY JR., H. J., Well-Test Analysis for Wells Producing from Two Commingled Zones of Unequal Thickness, Journal of Petroleum Technology, September 1974, p. $1035-1043$

[8] PEACEMAN, D. W., Interpretation of Well-Block Pressures in Numerical Reservoir Simulation, SPE Journal, June 1978, p. 183-194

[9] AGARWAL, R. G., A New Method to Account for Producing Time Effects When Drawdown Type Curves Are Used to Analyze Pressure Buildup and Other Test Data, Annual Fall Technical Conference and Exhibition of SPE, September 1980, p. 1-20

[10] GAO, C., Determination of Parameters for Individual Layers in Multilayer Reservoirs by Transient Well Tests, SPE Formation Evaluation, March 1987, p. 43-65 
[11] EHLIG-ECONOMIDES, C. A.; JOSEPH, J., A New Test for Determination of Individual Properties in a Multilayered Reservoir, SPE Formation Evaluation, September 1987, p. 261-283

[12] ABBASZADEH, M.; KAMAL, M., Pressure-Transient Testing of Water-Injection Wells, SPE Reservoir Engineering. February, 1989, p. 115-124

[13] BOURDET, D.; AYOUB, J. A.; PLRARD, Y. M., Use of Pressure Derivative in Well-Test Interpretation, SPE Formation Evaluation, June 1989 , p. $293-302$

[14] RAGHAVAN, R., Behavior of Wells Completed in Multiple Producing Zones, SPE Formation Evaluation, June 1989, p. 219-230

[15] PARK, H., Well Test Analysis of a Multilayered Reservoir with Formation Crossflow, Stanford University, PHD Thesis, 1989

[16] BRATVOLD, R. B.; HORNE, R. N., Analysis of Pressure-Falloff Tests Following Cold-Water Injection, SPE Formation Evaluation. September 1990 , p. 293-302

[17] OLIVEIRA, E. A. P.; SERRA, K. V., Oil/Water Relative Permeability Estimation from Falloff Tests, SPE Advanced Technology Series. 1994 Vol. 4 No 1, p. 59-66

[18] SPATH, J. B.; OZKAN, E.; RAGHAVAN, R. An Efficient Algorithm for Computation of Well Responses in Commingled Reservoirs, SPE Formation Evaluation, June, 1994, p. 115-121

[19] THOMPSON, L. G.; REYNOLDS, A. C., Well Testing for Radially Heterogeneous Reservoirs Under Single and Multiphase Flow Conditions, SPE Formation Evaluation, March 1997, p. 57-64

[20] BANERJEE, R.; THOMPSON, L. G., REYNOLDS, A. C., Injection/Falloff Testing in Heterogeneous Reservoirs, SPE Reservoir Evaluation \& Engineering. December, 1998, p. 519-527

[21] PERES, A. M. M.; REYNOLDS, A. C., Theory and Analysis of Injectivity Tests on Horizontal Wells, SPE Journal. June 2003, p. 148-159

[22] PERES, A. M. M.; BOUgharA, A. A.; CHEN, S.; MACHADO, A. A. V.; REYNOLDS, A. C., Approximate Analytical Solutions for the Pressure Response at a Water Injection Well, SPE Annual Technical Conference and Exhibition, September 2004, p. 1-17 
[23] CHEN, S.; LI, G.; PERES, A.; REYNOLDS, A. C., A Well Test for In-Situ Determination of Relative Permeability Curves, SPE Annual Techinical Conference and Exhibition. 2005, p. 1-16

[24] Peres, A. M. M.; BOUghraRA, A. A.; REYNOLS, A.C.; Rate Superposition for Generating Pressure Falloff Solutions, SPE Journal. September 2006, p. 364-374

[25] BARRETO JR, A.; PERES, A.; PIRES, A., Water Injectivity Tests on Multilayered Oil Reservoirs, Brasil Offshore Conference and Exhibition, 2011, p. 1-11

[26] ARABZAI, A.; HONMA, S., Numerical Simulation of the Buckley-Leverett Problem, Proceedings of the School of Engineering, Tokai University, Series E, may,2013, Vol 38, p. 9-14.

[27] SILVA, T. M. D., Modelagem Assistida de Lobos Turbidíticos Baseada em Dados de Teste de Formação, Pontifícia Universidade Católica do Rio de Janeiro (PUC-Rio), MS Thesis, 2017

[28] https://www.scilab.org/, acessed in October, 2017 
A

\section{Determining The Waterfront Shape}

In this appendix, will be presented the theory used to determine waterfront shape. First, the relation between fractional flow and mobility profile will be shown. Then, this relation will be applied along with Buckley-Leverett theory in order to achieve a means of calculating waterfront saturation profile. Last, a method to adjust the calculated waterfront profile will be developed.

\section{A.1}

\section{Fractional Flow}

Whenever a two-phase flow occurs, mass conservation ensures that total flow-rate is defined as the sum of the flow-rates from each phase:

$$
q=q_{1}+q_{2}
$$

If flow happens through a porous media, Darcy's law is applicable in a inclined control volume, as in figure A.1:

$$
q_{f}=-\frac{A k_{f}}{\mu_{f}} \frac{\partial\left(P_{f}+\rho_{f} g \sin (\alpha)\right)}{\partial x}
$$

where the subscript $f$ denotes the phase considered.

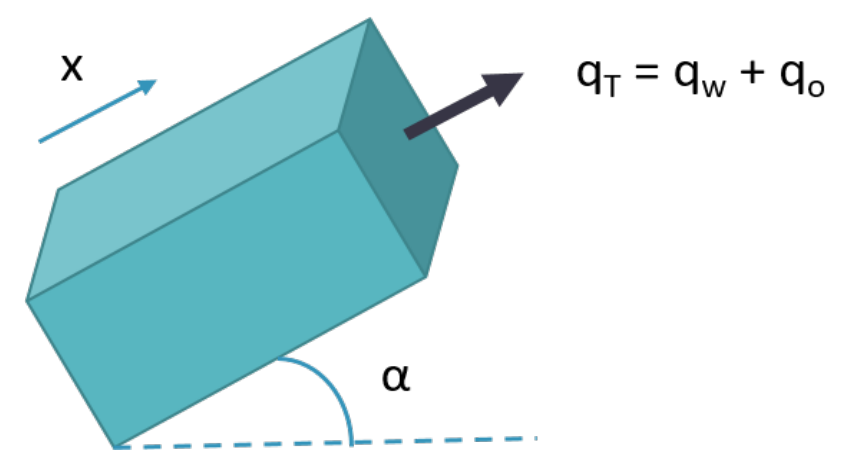

Figure A.1: Control Volume

Thus:

$$
q_{f}=-\frac{A k_{f}}{\mu_{f}}\left(\frac{\partial P_{f}}{\partial x}+\frac{\partial \rho_{f} g \sin (\alpha)}{\partial x}\right)
$$


Both the gravitational constant $g$ and angle $\alpha$ do not depend on the position. By hypothesis, fluids are slightly compressible. That means the density derivative is much smaller than the pressure derivative and, thereby, may be neglected:

$$
q_{f} \approx-\frac{A k_{f}}{\mu_{f}} \frac{\partial P_{f}}{\partial x}
$$

Capillary pressure consists on the difference between each phase's pressure. As it was assumed to be neglegible, a relation between the pressure derivatives from each phase is given by:

$$
P_{C}=P_{1}-P_{2} \approx 0 \Rightarrow \frac{\partial P_{1}}{\partial x}-\frac{\partial P_{2}}{\partial x}=0
$$

Hence, by equations (A-4) and (A-5):

$$
\frac{q_{1} \mu_{1}}{A k_{1}}-\frac{q_{2} \mu_{2}}{A k_{2}}=0
$$

Multiplying both sides by $k_{1} A / \mu_{1} q$ :

$$
\underbrace{\frac{q_{1}}{q}}_{f_{1}}-\underbrace{\frac{q_{2}}{q}}_{f_{2}} \frac{\mu_{2}}{k_{2}} \frac{k_{1}}{\mu_{1}}=0
$$

The flow-rates ratios on the equation above represent, respectively, the fractional flow for phases 1 and 2, i.e., the percentage of each phase flow in the total flow-rate inside a control volume. Thus, fractional flow for phase 1 is defined as:

$$
f_{1}=f_{2} \frac{\mu_{2}}{k_{2}} \frac{k_{1}}{\mu_{1}}
$$

And analogously for phase 2. But, by definition, the mobility of a phase is given by $k_{f} / \mu_{f}$. Therefore:

Since $f_{1}+f_{2}=1$ :

$$
f_{1}=f_{2} \frac{\lambda_{1}}{\lambda_{2}}
$$

$$
f_{1}=\left(1-f_{1}\right) \frac{\lambda_{1}}{\lambda_{2}}
$$

Multiplying both sides by $\lambda_{2}$ :

$$
f_{1} \lambda_{2}+f_{1} \lambda_{1}=\lambda_{1}
$$

Thus:

$$
f_{1}=\frac{\lambda_{1}}{\lambda_{1}+\lambda_{2}}=\frac{\lambda_{1}}{\lambda_{t}}
$$

And equation (A-12) shows that the fractional flow depends only on the mobility profile. 


\section{A. 2}

\section{Buckley-Leverett Theory For Cylindrical Geometry}

Buckley-Leverett theory is a conservation-based model used to analyze two-phase flow in a porous media. Therefore, this theory is useful to forecast the waterfront radius after a certain period of time, i.e., how far the injected water has swept the reservoir.

Model assumes that both fluids are immiscible and incompressible, and that flow is horizontal and unidimensional (or radial). Also, gravitational and capillary forces are neglected.

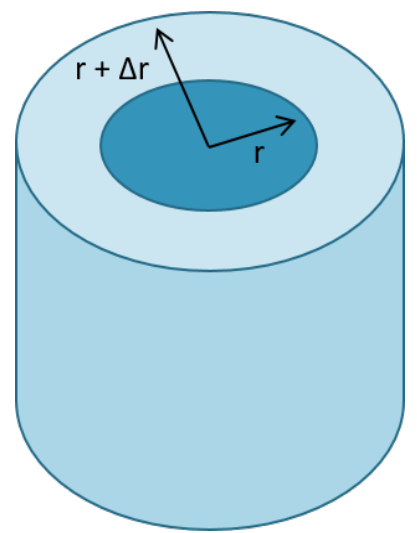

Figure A.2: Cylindrical Control Volume

Starting from a cylindrical control volume concentric with the wellbore as depicted in figure A.2, one may write the following mass balance:

$$
\text { Mass Inlet - Mass Outlet }=\text { Mass Accumulation }
$$

For a given instant of time, water mass inlet and outlet are defined as follows:

$$
\begin{array}{r}
\text { Mass Inlet }=\left(v_{w} \rho_{w} A\right)_{r} \Delta t=\left(v_{w} \rho_{w} 2 \pi r h\right)_{r} \Delta t \\
\text { Mass Outlet }=\left(v_{w} \rho_{w} A\right)_{r+\Delta r} \Delta t=\left(v_{w} \rho_{w} 2 \pi r h\right)_{r+\Delta r} \Delta t
\end{array}
$$

where $v_{w}, \rho_{w}$ and $A$ are, respectively, water velocity, water density and the cross-sectional area.

Subtracting equation (A-15) from equation (A-14):

$$
\text { Mass Inlet - Mass Outlet }=\rho_{w} \Delta t 2 \pi h\left(\left.r v_{w}\right|_{r}-\left.r v_{w}\right|_{r+\Delta r}\right)
$$

Mass in two consecutive time steps is evaluated as follows:

Mass at a given instant of time $=\left(\Delta r A \phi S_{w} \rho_{w}\right)_{t}=\left(\Delta r 2 \pi h \phi S_{w} \rho_{w}\right)_{t}$ 
Mass at the next instant of time $=\left(\Delta r A \phi S_{w} \rho_{w}\right)_{t+\Delta t}=\left(\Delta r 2 \pi h \phi S_{w} \rho_{w}\right)_{t+\Delta t}$

where $\phi$ stands for the rock porosity and $S_{w}$ is the water saturation for given time and radius.

Mass accumulation is calculated by the mass derivative with respect to time:

$$
\text { Mass accumulation }=\Delta r 2 \pi h \phi \rho_{w}\left(\left.r S_{w}\right|_{t+\Delta t}-\left.r S_{w}\right|_{t}\right)
$$

Applying equations (A-16) and (A-19) in the mass balance (A-13):

$$
\begin{gathered}
\rho_{w} \Delta t 2 \pi h\left(\left.r V_{w}\right|_{r}-\left.r V_{w}\right|_{r+\Delta r}\right)=2 \pi h \rho_{w} \Delta r \phi r\left(\left.S_{w}\right|_{t+\Delta t}-\left.S_{w}\right|_{t}\right) \\
\frac{\left(\left.r V_{w}\right|_{r}\right)-\left(\left.r V_{w}\right|_{r+\Delta r}\right)}{r \Delta r}=\phi \frac{\left(\left.S_{w}\right|_{t+\Delta t}\right)-\left(\left.S_{w}\right|_{t}\right)}{\Delta t}
\end{gathered}
$$

At the limit when $\Delta r$ and $\Delta t$ tend to zero, equation (A-21) may be rewritten as:

$$
\Rightarrow-\frac{1}{r} \frac{\partial}{\partial r}\left(r V_{w}\right)=\phi \frac{\partial S_{w}}{\partial t}
$$

The deduction for the oil-phase is analogous.

For each position and each timestep, the process is assumed to be stationary. Under such circumstances, saturation derivative with respect to radius is related to saturation derivative with respect to time by the following expression (1):

$$
\frac{\partial S_{w}}{\partial t}=-\frac{\partial S_{w}}{\partial t} \frac{\partial t}{\partial r}
$$

Combining equations (A-22) and (A-23):

$$
\frac{1}{r} \frac{\partial}{\partial r}\left(r V_{w}\right)=\phi \frac{\partial S_{w}}{\partial r} \frac{\partial r}{\partial t}
$$

Multiplying the equation above by $2 \pi h$ :

$$
\frac{1}{r} \frac{\partial}{\partial r} q_{w}=\phi 2 \pi h \frac{\partial S_{w}}{\partial r} \frac{\partial r}{\partial t}
$$

It is now useful to write the water flow-rate in terms of water fractional flow and total injected flow-rate. As shown in appendix A.1, water fractional flow is given by:

$$
f_{w}=\frac{\frac{k_{w}}{\mu_{w}}}{\frac{k_{w}}{\mu_{w}}+\frac{k_{o}}{\mu_{o}}}=\frac{\lambda_{w}}{\lambda_{t}}
$$

If the relative permeability curve is known, it is possible to build a fractional flow curve as a function of water saturation. Thereby, water flow in equation (A-25) might be evaluated as: 


$$
q_{w}=f_{w} q
$$

Thus, the water flow derivative (A-25) might be calculated as a function of water fractional flow instead of water saturation:

$$
\frac{1}{r} \frac{\partial}{\partial r}\left(f_{w} q\right)=\frac{q}{r} \frac{\partial}{\partial r} f_{w}=\frac{q}{r} \underbrace{\frac{\partial f_{w}}{\partial S_{w}}}_{f_{w}^{\prime}} \frac{\partial S_{w}}{\partial r}
$$

Combining equations (A-25) and (A-28):

$$
\frac{q}{r} f_{w}^{\prime}=\phi 2 \pi h \frac{\partial r}{\partial t} \Rightarrow \frac{q f_{w}^{\prime}}{2 \pi h \phi} \partial t=r \partial r
$$

Integrating the two sides of the equation:

$$
\frac{q f_{w}^{\prime}}{2 \pi h \phi} t=\int_{r_{w}}^{r_{F}} r \partial r=\frac{r_{F}^{2}-r_{w}^{2}}{2}
$$

Therefore, waterfront radius for cylindrical geometry in a given time $t$ is computed as:

$$
r_{F}(t)=\sqrt{\frac{q f_{w}^{\prime} t}{\pi h \phi}+r_{w}^{2}}
$$

\section{A.3}

\section{Welge Method To Adjust The Waterfront Saturation Profile}

As equation (A-31) shows, waterfront radius calculated according to Buckley-Leverett theory depends on the fractional flow derivative and, hence, on the relative permeability curves (see figure A.3). Thus, water-oil interface along the waterfront is shaped by relative permeability data.
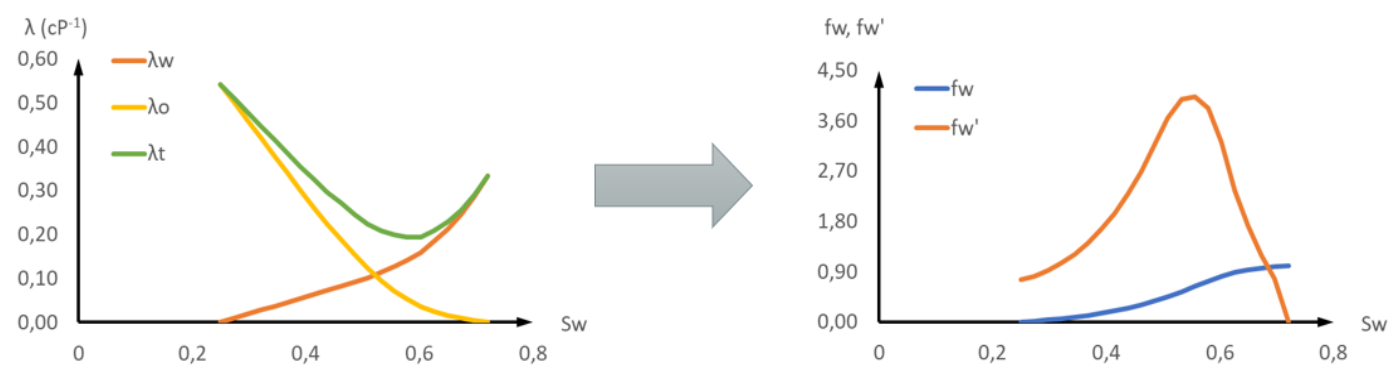

Figure A.3: Mobilities Profiles And Its Corresponding Fractional Flow Curve

Since fractional flow relies only on water-oil interaction, the derivative curve may assume a wide range of shapes. This may lead to a waterfront inconsistent with the physical nature of the problem, as gravity implies that the higher density fluid present higher saturation at the bottom of the reservoir. 
Such situation happens whenever the two-phase flow is more favorable to water than to oil.
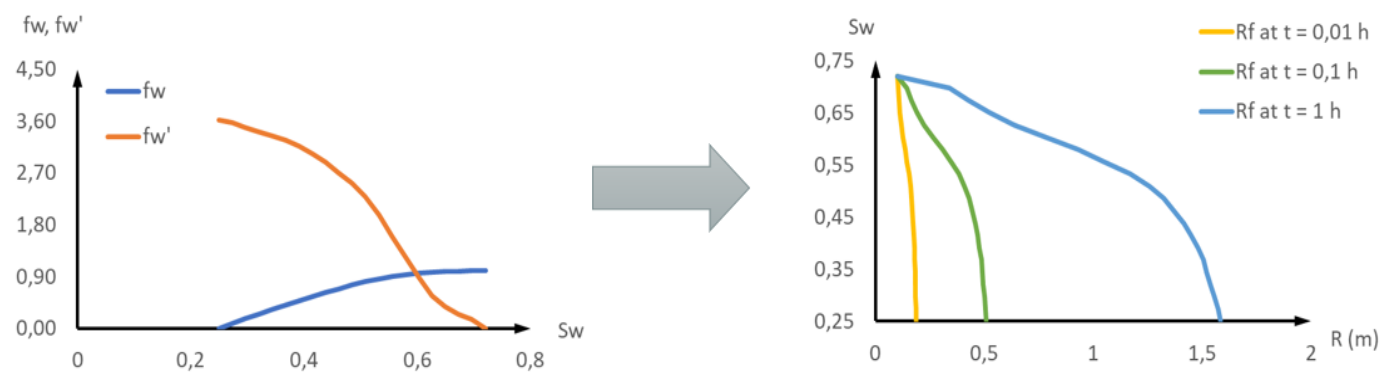

Figure A.4: Fractional Flow Curve And Its Corresponding Waterfront Profile

In figure A.4, the fractional derivative shape is typical for mobility ratio higher than 1, while figure A.5 depicts flow unfavorable to water. However, the behavior depicted in figure A.5 is inconsistent with the physical reality. Since permeability is assumed to be radially constant, it is not possible that a point further from the wellbore presents higher water saturation than one closer. Besides, saturation profile in figure A.5 implies that two different saturations might occur at the same radius, which is clearly impossible.
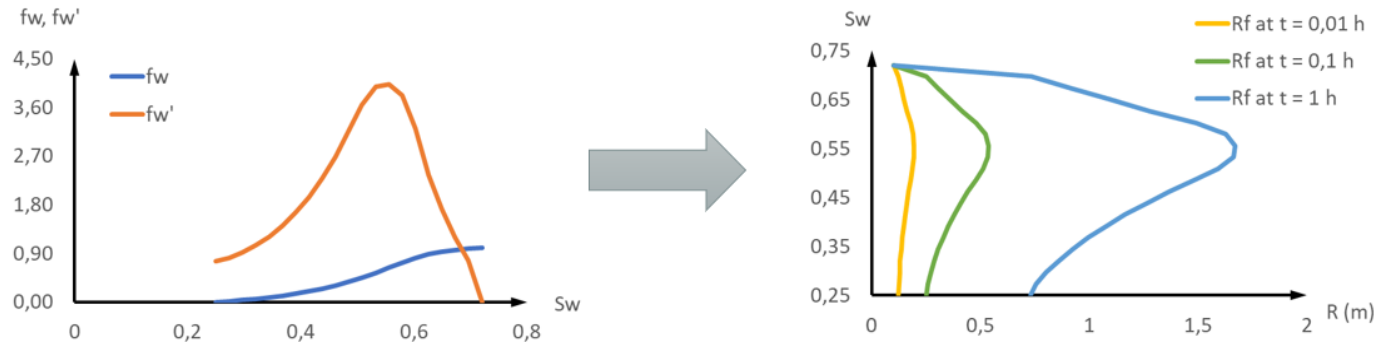

Figure A.5: Fractional Flow Curve And Resulting Inconsistent Waterfront Profile

Thus, whenever the fractional flow data present an inflection point, an adjustment is required, so that the estimated waterfront makes physical sense. This correction is achieved through Welge method for waterfront calculation.

This technique aims to build a straight line that starts at the connate water saturation and is tangent to the fractional flow curve (17). For all saturations bellow this tangency point is (denoted by $S_{w F}$ ), fractional flow derivative is assumed to be constant and equal to $f_{w}^{\prime}\left(S_{w F}\right)$.

The tangency point is determined by taking the highest saturation whose corresponding derivative enables the construction of a straight line that, simultaneously, is tangent to the fractional flow curve, passes through the 
reservoir initial water saturation and results in higher fractional flow values than the original curve.
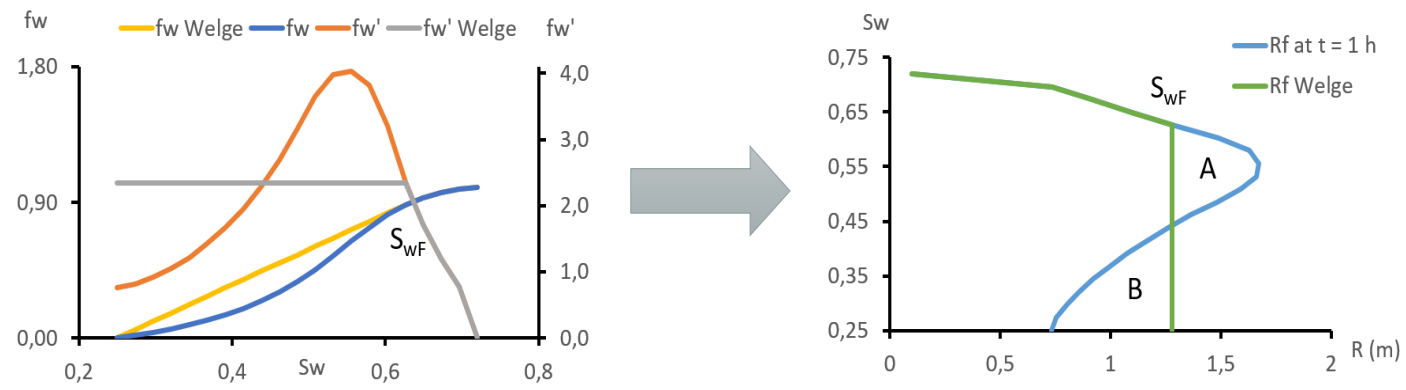

Figure A.6: Adjusted Fractional Flow Curve And Waterfront

Figure A.6 shows the adjusted fractional flow curve And Its Corresponding waterfront. Definition of $S_{w F}$ implies that areas $A$ and $B$ must be equal. This condition is required to ensure mass conservation along the adjusted waterfront (26). Thus, waterfront adjusted by Welge method is capable of describing two-phase flow in a consistent way. 
B

\section{Pressure Response In Single-Layer Reservoirs Under Single-Phase Flow}

Single-phase oil flow inside a reservoir is well known $(4,5,7,10,11)$. The following formulation was originally developed for production tests, during which oil is removed from the reservoir, causing a pressure drop. However, for a matter of convenience, it will be considered an injectivity test, assuming the injection fluid is the same oil existent inside the reservoir. All equations remain essentially the same, only difference is the flow-rate sign. If it is positive, then oil is removed from the reservoir; whereas negative flow-rates stand for injectivity tests.

The pressure change caused by such flow is depicted by a partial differential equation problem, given appropriate initial and boundary conditions. The same assumptions over the reservoir model made in chapter 3 are also valid. For a single-phase oil flow inside a single-layer reservoir under infinite radial flow, the mathematical formulation of the problem is given by (11):

Partial Differential Equation:

$$
\frac{1}{r} \frac{\partial}{\partial r}\left(r \frac{\partial \Delta P}{\partial r}\right)=\frac{1}{\eta} \frac{\partial \Delta P}{\partial t},
$$

where the term $\Delta P$ stands for the difference between initial and current pressure and the $\eta_{j}$ parameter represents the reservoir hydraulic diffusivity, defined as (10):

$$
\eta_{j}=\frac{k_{j}}{\phi_{j} \mu c_{t}}
$$

where $\phi_{j}$ denotes the porosity in layer $j$ and $c_{t}$ is the total compressibility.

Equation (B-1) describes how pressure inside any single-layer reservoir behaves after a given time interval. It is natural to infer that reservoirs presenting distinct initial conditions will present distinct pressure responses. Thus, a starting condition must be properly prescribed. For the reservoir model considered, the initial condition follows from the hypothesis that the reservoir is in equilibrium at the instant $t=0$ :

Initial Condition: 
Appendix B. Pressure Response In Single-Layer Reservoirs Under Single-Phase Flow

$$
\Delta P(r, t=0)=0
$$

To ensure uniqueness of the solution, it is also imperative to understand how the flow develops at the wellbore and at the reservoir boundaries. Outer boundary condition is a consequence of the reservoir's infinite extension. At a sufficiently large radius, wellbore presence is not felt by the reservoir. Hence, for any time, pressure at such radius is equal to the initial pressure. Thus:

Outer Boundary Condition:

$$
\lim _{r \rightarrow \infty} \Delta P(r, t)=0
$$

Inner boundary condition derives from Darcy's law.

Inner Boundary Condition:

$$
q=\frac{2 \pi k h}{\mu}\left(r \frac{\partial \Delta P}{\partial r}\right)_{r=r_{w}}
$$

Now, the problem depicted by equations (B-1), (B-3), (B-4) and (B-5) is well posed, and uniqueness is assured. One means for obtaining the analytical solution for the injection period is through the Laplace transform. Transforming equation (B-1) and using the initial condition (B-3), one gets:

$$
\frac{1}{r} \frac{\partial}{\partial r}\left(r \frac{\partial \overline{\Delta P}}{\partial r}\right)=\frac{1}{\eta} u \overline{\Delta P}
$$

where $u$ stands for the Laplace variable.

Applying the product rule at the partial derivative, equation (B-6) might be rewritten as:

$$
\frac{\partial^{2} \overline{\Delta P}}{\partial r^{2}}+\frac{1}{r} \frac{\partial \overline{\Delta P}}{\partial r}=\frac{u}{\eta} \overline{\Delta P}
$$

Rearranging equation (B-7) in a convenient manner:

$$
\frac{\partial^{2} \overline{\Delta P}}{\partial(r \sqrt{u / \eta})^{2}}+\frac{1}{r \sqrt{u / \eta}} \frac{\partial \overline{\Delta P}}{\partial(r \sqrt{u / \eta})}-\overline{\Delta P}=0
$$

Equation (B-8) is similar to the following equation:

$$
\frac{\partial}{\partial r}\left(\frac{\partial^{2} y}{\partial z^{2}}\right)+\frac{1}{z}\left(\frac{\partial y}{\partial z}\right)-\left(1-\frac{v^{2}}{z^{2}}\right)=0,
$$

that has as general solution (6):

$$
y=a I_{v}(z)+b K_{v}(z)
$$

where $a$ and $b$ are constants; $I_{v}$ is modified Bessel function of first kind and order $v$; and $K_{v}$ is modified Bessel function of second kind and order $v$.

Thus, equation (B-8) has as general solution:

$$
\overline{\Delta P}=a I_{0}\left(r \sqrt{\frac{u}{\eta}}\right)+b K_{0}\left(r \sqrt{\frac{u}{\eta}}\right)
$$


In order to determine the constants, the Laplace transform must be applied to the boundary conditions. Transforming the OBC (B-4) to Laplace domain:

$$
\lim _{r \rightarrow \infty} \overline{\Delta P}(r, t)=\lim _{r \rightarrow \infty}\left[a I_{0}\left(r \sqrt{\frac{u}{\eta}}\right)+b K_{0}\left(r \sqrt{\frac{u}{\eta}}\right)\right]
$$

Since the Bessel functions are sufficiently regular, the limit of sum is equal to the sum of limits:

$$
\lim _{r \rightarrow \infty} \overline{\Delta P_{j}}(r, t)=a \lim _{r \rightarrow \infty} I_{0}\left(r \sqrt{\frac{u}{\eta_{j}}}\right)+b \lim _{r \rightarrow \infty} K_{0}\left(r \sqrt{\frac{u}{\eta_{j}}}\right)
$$

Owing to the limit of the Bessel function (6), it is easy to notice that $a=0$. Constant $\mathrm{b}$ is computed by transforming the IBC (B-5):

$$
\frac{q}{u}=-\frac{2 \pi k h r}{\mu}\left(\frac{\partial \overline{\Delta P}}{\partial r}\right)_{r_{w}}=-\frac{2 \pi k h r}{\mu} \frac{\partial\left(b K_{0}\left(r \sqrt{\frac{u}{\eta}}\right)\right)}{\partial r}
$$

Using the property of the Bessel function derivative (6):

$$
\frac{q}{u}=\frac{2 \pi k h r_{w}}{\mu} b \sqrt{\frac{u}{\eta}} K_{1}\left(r_{w} \sqrt{\frac{u}{\eta}}\right)
$$

Thereby, the constant $\mathrm{b}$ is given by:

$$
b=\frac{q \mu}{2 \pi u k h r_{w} \sqrt{u / \eta}} \frac{1}{K_{1}\left(r_{w} \sqrt{u / \eta}\right)}
$$

Thus, the pressure in Laplace domain is calculated by the following expression:

$$
\overline{\Delta P}(r, t)=b K_{0}\left(r \sqrt{\frac{u}{\eta}}\right)=\frac{q \mu}{2 \pi u k h r_{w} \sqrt{u / \eta}} \frac{K_{0}\left(r_{w} \sqrt{u / \eta}\right)}{K_{1}\left(r_{w} \sqrt{u / \eta}\right)}
$$

Expression (B-17) may be easily converted to the real field through the Stehfest algorithm (2). 


\section{C \\ Pressure Response In Multilayer Reservoirs Under Single-Phase Flow}

Formulation for multilayer reservoirs is an extension of the one presented in the previous section. Here once again, the flow-rate sign will point whether an injectivity or production test is considered.

Now, the partial differential equation describes pressure change in an arbitrary layer $j(11)$ :

Partial Differential Equation:

$$
\frac{1}{r} \frac{\partial}{\partial r}\left(r \frac{\partial \Delta P_{j}}{\partial r}\right)=\frac{1}{\eta_{j}} \frac{\partial \Delta P_{j}}{\partial t}
$$

where the term $\Delta P_{j}$ stands for the difference between initial and current pressure in layer $j$ and the $\eta_{j}$ parameter represents the hydraulic diffusivity in layer $j$, defined as (10):

$$
\eta_{j}=\frac{k_{j}}{\phi_{j} \mu c_{t}}
$$

Initial and boundary conditions are also adapted from the single-layer case (11):

Initial Condition:

$$
\Delta P_{j}(r, t=0)=0
$$

Outer Boundary Condition:

$$
\lim _{r \rightarrow \infty} \Delta P_{j}(r, t)=0
$$

Inner Boundary Condition:

$$
q_{j}=\frac{2 \pi}{\mu} k_{j} h_{j}\left(r \frac{\partial \Delta P}{\partial r}\right)_{r=r_{w}}
$$

The analytical solution for the injection period is obtained through the Laplace transform. Transformation of equation (C-1) yields (using the initial condition (C-3)):

$$
\frac{1}{r} \frac{\partial}{\partial r}\left(r \frac{\partial \overline{\Delta P_{j}}}{\partial r}\right)=\frac{1}{\eta_{j}} u \overline{\Delta P_{j}}
$$

Applying the product rule at the partial derivative, equation (C-6) might be rewritten as: 


$$
\frac{\partial^{2} \overline{\Delta P_{j}}}{\partial r^{2}}+\frac{1}{r} \frac{\partial \overline{\Delta P_{j}}}{\partial r}=\frac{u}{\eta_{j}} \overline{\Delta P_{j}}
$$

Rearranging equation (C-7) in a convenient manner:

$$
\frac{\partial^{2} \overline{\Delta P_{j}}}{\partial\left(r \sqrt{u / \eta_{j}}\right)^{2}}+\frac{1}{r \sqrt{u / \eta_{j}}} \frac{\partial \overline{\Delta P_{j}}}{\partial\left(r \sqrt{u / \eta_{j}}\right)}-\overline{\Delta P_{j}}=0
$$

Thus, equation (C-8) has as general solution (6):

$$
\overline{\Delta P_{j}}=a I_{0}\left(r \sqrt{\frac{u}{\eta_{j}}}\right)+b K_{0}\left(r \sqrt{\frac{u}{\eta_{j}}}\right)
$$

The constants $a$ and $b$ are computed by applying the Laplace transform to the boundary conditions. Transforming the OBC to Laplace domain:

$$
\lim _{r \rightarrow \infty} \overline{\Delta P_{j}}(r, t)=\lim _{r \rightarrow \infty}\left[a I_{0}\left(r \sqrt{\frac{u}{\eta_{j}}}\right)+b K_{0}\left(r \sqrt{\frac{u}{\eta_{j}}}\right)\right]
$$

Writing the limit of sum as the sum of limits:

$$
\lim _{r \rightarrow \infty} \overline{\Delta P_{j}}(r, t)=a \lim _{r \rightarrow \infty} I_{0}\left(r \sqrt{\frac{u}{\eta_{j}}}\right)+b \lim _{r \rightarrow \infty} K_{0}\left(r \sqrt{\frac{u}{\eta_{j}}}\right)
$$

The limit of Bessel functions ensures that $a=0$ once again (6).

Transforming the IBC:

$$
\frac{q_{j}}{u}=\frac{2 \pi k_{j} h_{j} r_{w}}{\mu} b \sqrt{\frac{u}{\eta_{j}}} K_{1}\left(r_{w} \sqrt{\frac{u}{\eta_{j}}}\right)
$$

Thereby, it is possible to compute constant b:

$$
b=\frac{q_{j} \mu}{2 \pi u k_{j} h_{j} r_{w} \sqrt{u / \eta_{j}}} \frac{1}{K_{1}\left(r_{w} \sqrt{u / \eta_{j}}\right)}
$$

Thus, the pressure in layer $j$ is calculated in Laplace domain by the following expression:

$$
\overline{\Delta P_{j}}(r, t)=b K_{0}\left(r \sqrt{\frac{u}{\eta_{j}}}\right)=\frac{q_{j} \mu}{2 \pi u k_{j} h_{j} r_{w} \sqrt{u / \eta_{j}}} \frac{K_{0}\left(r_{w} \sqrt{u / \eta_{j}}\right)}{K_{1}\left(r_{w} \sqrt{u / \eta_{j}}\right)}
$$

Isolating the flow-rate in layer $j$ from equation (C-14):

$$
q_{j}=\overline{\Delta P_{j}}(r, t) \frac{2 \pi u k_{j} h_{j} r_{w}}{\mu} \sqrt{\frac{u}{\eta_{j}}} \frac{K_{1}\left(r_{w} \sqrt{u / \eta_{j}}\right)}{K_{0}\left(r_{w} \sqrt{u / \eta_{j}}\right)}
$$

Total injection flow-rate is evaluated by summing the flow-rates in every layer:

$$
q_{i n j}=\sum_{j=1}^{n} q_{j}=\sum_{j=1}^{n} \overline{\Delta P_{j}}(r, t) \frac{2 \pi u k_{j} h_{j} r_{w}}{\mu} \sqrt{\frac{u}{\eta_{j}}} \frac{K_{1}\left(r_{w} \sqrt{u / \eta_{j}}\right)}{K_{0}\left(r_{w} \sqrt{u / \eta_{j}}\right)}
$$


However, by hypothesis, pressure rise is the same for all layers, which implies that:

$$
\overline{\Delta P_{1}}=\overline{\Delta P_{2}}=\ldots=\overline{\Delta P_{n}}=\overline{\Delta P}
$$

Therefore, equation (C-16) might be rewritten as:

$$
\overline{\Delta P}=\frac{1}{\sum_{j=1}^{n} \frac{2 \pi u k_{j} h_{j} r_{w}}{q_{i n j} \mu} \sqrt{\frac{u}{\eta_{j}}} \frac{K_{1}\left(r_{w} \sqrt{u / \eta_{j}}\right)}{K_{0}\left(r_{w} \sqrt{u / \eta_{j}}\right)}}
$$

Rearranging equation (C-18):

$$
\overline{\Delta P}=\frac{q_{i n j} \mu}{2 \pi r_{w} \sum_{j=1}^{n} u k_{j} h_{j} \sqrt{\frac{u}{\eta_{j}}} \frac{K_{1}\left(r_{w} \sqrt{u / \eta_{j}}\right)}{K_{0}\left(r_{w} \sqrt{u / \eta_{j}}\right)}}
$$

Finally, Stehfest algorithm is once again used to convert expression (C-19) to the real field (2). It is also clear that, whenever equation (C-19) is applied to single-layer reservoirs, it reduces to (B-17), as expected. 
D

\section{Superposition Principle}

The linearity of partial differential equation (B-1) enables the usage of the superposition principle to describe flow-rate history of a single-phase flow. That is, if $f$ and $g$ are solutions of the PDE, then $A f+B g$ is also a solution for the same PDE ( $A$ and $B$ are constants). Thus, the conventional flow history during a single-phase flow may be depicted by the superposition principle as well. After shut-in, flow-rate becomes zero.

This is equivalent to a test with two wells at the same point of the reservoir $(10,24)$ : one that starts injecting fluid at the initial time, and the other with equal flow-rate, but with opposite sign, that starts at the instant the well is shut $\left(t_{p}\right)$. Figure D.1 shows how the flow-rate history is represented according to the superposition principle.
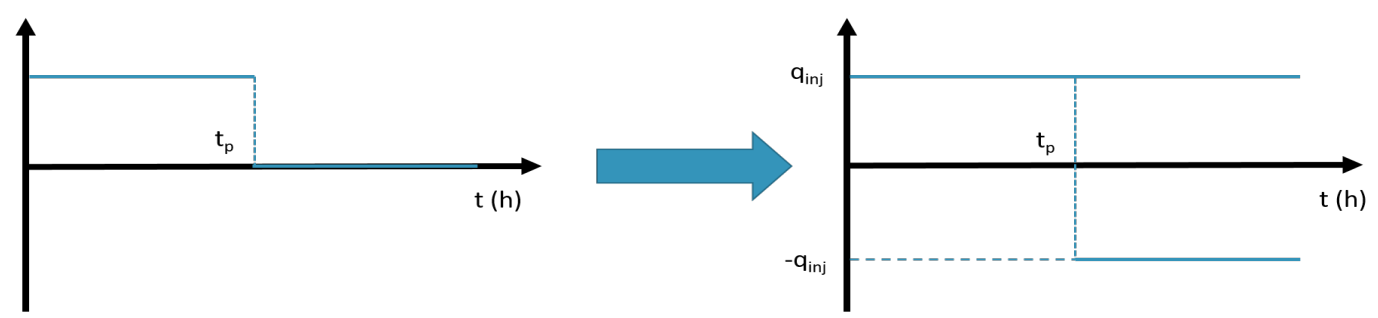

Figure D.1: Flow History And Superposition Equivalent Scheme

Therefore, the calculation of well bottom hole pressure is made essentially by computing the contribution from each well:

$$
\Delta P_{w s}\left(t_{p}+\Delta t\right)=\Delta P_{w f}\left(t_{p}+\Delta t\right)+\Delta P_{w f}(\Delta t),
$$

and each term is evaluated as shown in appendixes $\mathrm{C}$ and $\mathrm{B}$. Thus, from equations (C-19) and (D-1), pressure measured at the wellbore at any instant after the well is shut is computed as: 


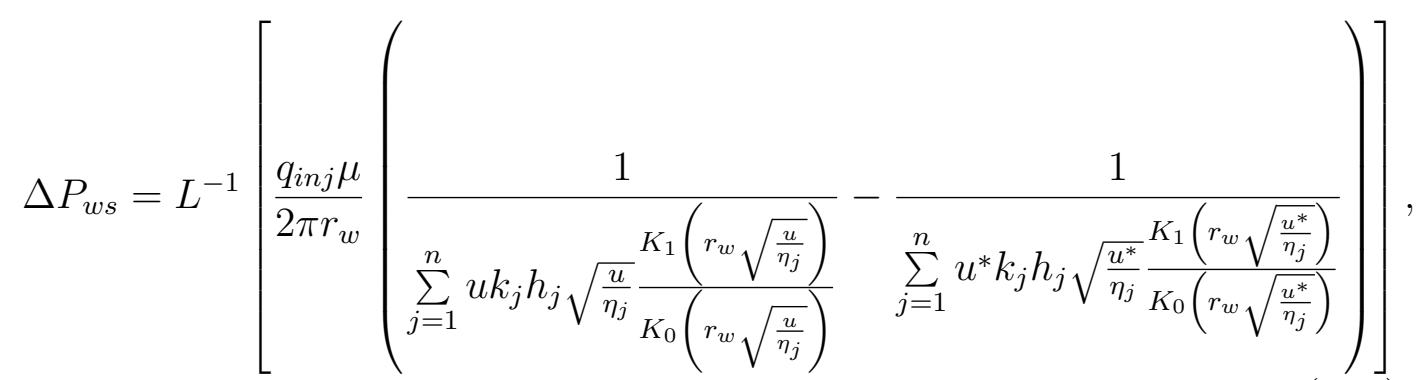

where $u$ and $u^{*}$ are the Laplace variables associated to each flow-rate displayed in figure D.1. The ratio between $u$ and $u^{*}$ is given by:

$$
u^{*}=u \frac{t_{p}+\Delta t}{\Delta t}
$$

